Supporting Information

\title{
Applying Green Metrics to Eco-Friendly Synthesis of Sulfur- Substituted Conjugated Dienes Based on Atom-Economic Hydrothiolation
}

\author{
Evgeniya S. Degtyareva, Evgeniya V. Borkovskaya, Valentine P. Ananikov* \\ Zelinsky Institute of Organic Chemistry, Russian Academy of Sciences, Leninsky \\ Prospect 47, Moscow 119991, Russia; http://AnanikovLab.ru \\ e-mail: val@ioc.ac.ru
}

Total number of pages: 47 (S1-S47)

Total number of schemes: 6 (S1-S6)

Total number of figures: $\mathbf{2 8}$ (S1-S28)

Total number of tables: $\mathbf{3 4}$ (S1-S34)

Total number of equations $\mathbf{8 9}$ (S1-S89) 


\section{Contents}

General information 3

1. Comparison of the environmental impacts of different methods of 2a synthesis 3

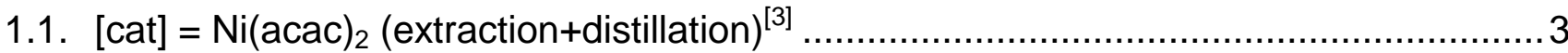

1.2. $[\mathrm{cat}]=\mathrm{Ni}(\mathrm{acac})_{2}$ (flash chromatography) …..................................................... 4

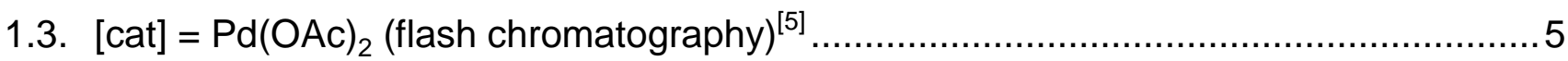

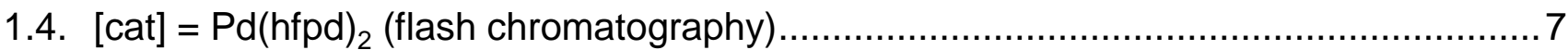

1.5. $[\mathrm{cat}]=(\mathrm{IMes}) \mathrm{Pd}(\mathrm{acac}) \mathrm{Cl}(\text { dry column vacuum chromatography })^{[11]} \ldots \ldots \ldots \ldots \ldots \ldots \ldots \ldots . . . . . . . . . . .11$

2. $\mathrm{EF}$ and $\mathrm{CEF}$ values in the synthesis of $2 \mathrm{a}$, purified by distillation ................................18

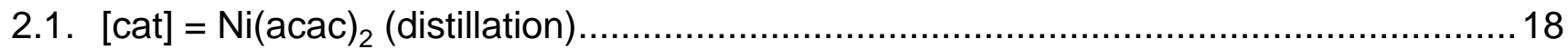

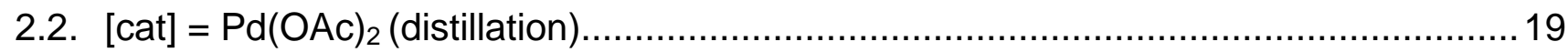

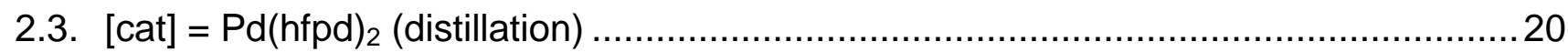

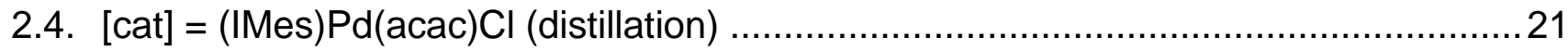

3. Dehydration of vinyl sulfides to sulfur-functionalized 1,3-butadienes ...........................23

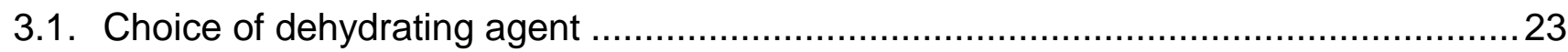

3.2. Evaluation of $\mathrm{CEF}$ for vinyl sulfides dehydration ..................................................25

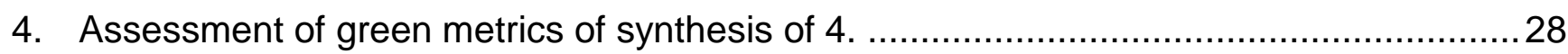

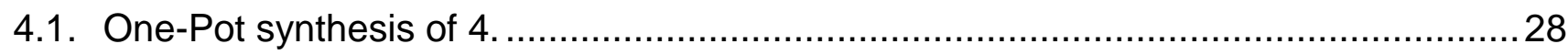

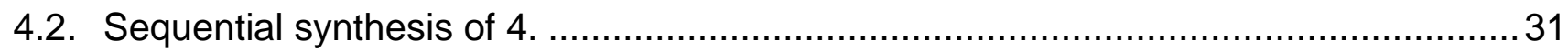

NMR spectra of dehydration reaction and Diels-Alder reaction products .............................33

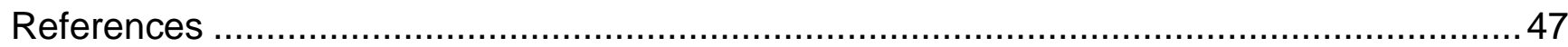




\section{General information}

The calculations were carried out according to the literature data, since any of the method contains information about solvent recyclability, $90 \%$ of solvents recyclability was assumed. ${ }^{[1,2]}$ All the raw materials should be available from the commercial sours and their price should not exceed $\$ 100 / \mathrm{mol}^{[2]}$

\section{Comparison of the environmental impacts of different methods of $2 a$ synthesis}

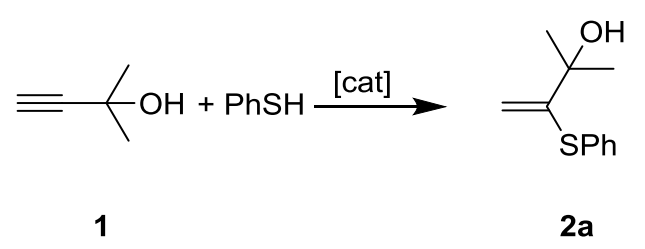

Scheme S1. Thiophenol addition to 2-methyl-3-butyn-2-ol.

\section{1. $\quad[$ cat $]=\mathrm{Ni}(\mathrm{acac})_{2}(\text { extraction+distillation })^{[3]}$}

The calculations were made according to the published procedure [3]: "Ni(acac) $)_{2}(0.2$ $\mathrm{mmol}, 0.0514 \mathrm{~g})$ and 2-methyl-3-butyn-2-ol (1) $(10 \mathrm{mmol}, 0.977 \mathrm{~mL})$ were mixed in a test tube at room temperature to form a uniform green suspension. Under continuous stirring in a water/ice bath at $5^{\circ} \mathrm{C}, \mathrm{PhSH}(20 \mathrm{mmol}, 2.054 \mathrm{~mL})$ was slowly added. The color of the suspension immediately changed from green to dark. The test-tube was flushed with argon and closed. To avoid overheating, the reaction mixture was cooled for additional $10 \mathrm{~min}$. The reaction was carried out at $40{ }^{\circ} \mathrm{C}$ for $30 \mathrm{~min}$.

After completion of the reaction, nickel-containing species were filtered from the reaction mixture through Celite using $\mathrm{CH}_{2} \mathrm{Cl}_{2}$ as an eluent $(40 \mathrm{~mL})$ to obtain a transparent homogeneous solution. To remove the excess of $\mathrm{PhSH}$, aqueous $\mathrm{NaOH}(25 \mathrm{~mL}, 5 \mathrm{M}$ ) was added, and the remaining organic phase was extracted with hexane. The organic layer was dried over $\mathrm{Na}_{2} \mathrm{SO}_{4}$, and the solvent was removed under reduced pressure. 2-(Phenylthio)-3hydroxy-3-methyl-1-butene 2a was obtained as a light-brown liquid in $80 \%$ yield $(1.55 \mathrm{~g})$; after the distillation, it was obtained as a light-yellow liquid in $55 \%$ yield $(1.07 \mathrm{~g})$." 
Table S1. Material Input-Output for the $\mathrm{Ni}\left(\mathrm{acac}_{2}\right)_{2}$-Catalyzed Hydrothiolation with Product Purification by Extraction and Distillation.

\begin{tabular}{|c|c|c|c|c|c|c|c|c|c|}
\hline Step & $\begin{array}{c}\text { Input } \\
\text { material }\end{array}$ & $\begin{array}{l}\text { Input } \\
\text { type }\end{array}$ & $\begin{array}{l}\text { Output } \\
\text { Material }\end{array}$ & $\begin{array}{c}\mathrm{Mw} \\
(\mathrm{g} / \mathrm{mol})\end{array}$ & $\begin{array}{l}\mathrm{D}, \\
\mathrm{g} / \mathrm{mL}\end{array}$ & Equiv. & $\begin{array}{l}\text { Input } \\
\text { (mol) }\end{array}$ & $\begin{array}{c}\text { Input } \\
\text { Weight }\end{array}$ & $\begin{array}{l}\text { Output } \\
\text { Weight }\end{array}$ \\
\hline \multirow{3}{*}{ 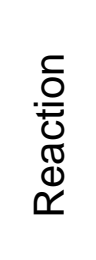 } & 1 & $\begin{array}{c}\text { Raw } \\
\text { material }\end{array}$ & & 84.12 & & 1 & 0.01 & 0.841 & \\
\hline & $\mathrm{PhSH}$ & $\begin{array}{c}\text { Raw } \\
\text { material }\end{array}$ & & 110.17 & & 2 & 0.02 & 2.203 & \\
\hline & $\mathrm{Ni}(\text { acac })_{2}$ & Catalyst & & 256.9 & & 0.02 & 0.0002 & 0.05 & \\
\hline \multirow{7}{*}{ 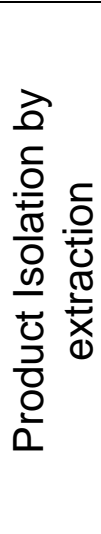 } & Celite & Workup & & & & & & $2.5^{[\mathrm{a}]}$ & \\
\hline & $\mathrm{CH}_{2} \mathrm{Cl}_{2}$ & Solvent & & & 1.33 & & & 53.20 & \\
\hline & Hexane & Solvent & & & 0.66 & & & 13.20 & \\
\hline & $\mathrm{NaOH}$ & Workup & & 40 & & & 0.125 & 5 & \\
\hline & Water & Solvent & & 18.02 & & & & $24.63^{[b]}$ & \\
\hline & $\mathrm{Na}_{2} \mathrm{SO}_{4}$ & Workup & & 142.04 & & & 0.0007 & $0.099^{[c]}$ & \\
\hline & & Product & $2 a$ & & & & & & 1.55 \\
\hline \multirow{2}{*}{ 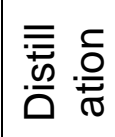 } & $2 a$ & Product & & 194.29 & & & & 1.55 & \\
\hline & & Product & $2 a$ & & & & & & 1.07 \\
\hline
\end{tabular}

[a] Amount of silica gel was taken based on Celite load in $60 \mathrm{~mL}$ filter funnel (sigmaaldrich.com cat. number CHR104860). ${ }^{[b]}$ The weight of the water for $5 \mathrm{M} \mathrm{NaOH}$ solution was calculated based on $\mathrm{d}(5 \mathrm{M} \mathrm{NaOH})=1.185 \mathrm{~g} / \mathrm{mL} .{ }^{[\mathrm{c}]}$ Based on water solubility in $\mathrm{CH}_{2} \mathrm{Cl}_{2} 0.198 \mathrm{~g}$ in $100 \mathrm{~g}$ and water solubility in Hexane $0.099 \mathrm{~g}$ in $100 \mathrm{~g}(\Sigma 0.118 \mathrm{~g})$.

$$
\begin{gathered}
E F=\frac{0.841+2.203+0.05+2.5+53.2 \times 0.1+13.2 \times 0.1+5+0.10-1.07}{1.07}=15.2 \\
c E F=\frac{0.84+2.203+0.05+2.5+53.2+13.2+5+24.63+0.10-1.07}{1.07}=94.1 \\
\text { SEF reaction }=\frac{0.84+2.20+0.05-1.55}{1.55}=1.0
\end{gathered}
$$

\section{2. $[$ cat $]=\mathrm{Ni}(\text { acac })_{2}$ (flash chromatography $)^{[4]}$}

The calculations were made according to the published procedure [4]: "Ni(acac $)_{2}(0.1$ $\mathrm{mmol}, 0.0260 \mathrm{~g})$ and 2-methyl-3-butyn-2-ol (1) $(1.25 \mathrm{mmol}, 1.122 \mathrm{~mL})$ were mixed at room temperature to form a uniform green suspension. The suspension was cooled to $5{ }^{\circ} \mathrm{C}$ in an ice bath, and $\mathrm{PhSH}(5 \mathrm{mmol}, 0.55 \mathrm{~g}$ ) was slowly added under continuous stirring. The tube was flushed with argon and closed. The rest of the alkyne $(3.75 \mathrm{mmol}, 0.366 \mathrm{~mL})$ was added dropwise over 40 min with a syringe to the reaction mixture under continuous stirring at 
$40^{\circ} \mathrm{C}$. After completion of the reaction, the product was isolated by flash chromatography on silica gel."

Table S2. Material Input-Output for the $\mathrm{Ni}(\mathrm{acac})_{2}$-Catalyzed Hydrothiolation. Product Purification by Flash Chromatography.

\begin{tabular}{|c|c|c|c|c|c|c|c|c|}
\hline Step & $\begin{array}{c}\text { Input } \\
\text { material }\end{array}$ & Input type & $\begin{array}{c}\text { Output } \\
\text { Materia } \\
\text { I }\end{array}$ & $\begin{array}{c}\mathrm{Mw} \\
(\mathrm{g} / \mathrm{mol})\end{array}$ & Equiv. & $\begin{array}{l}\text { Input } \\
\text { (mol) }\end{array}$ & $\begin{array}{c}\text { Input } \\
\text { Weight }\end{array}$ & $\begin{array}{l}\text { Output } \\
\text { Weight }\end{array}$ \\
\hline \multirow{3}{*}{ 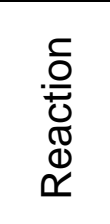 } & $\mathrm{Ni}(\text { acac })_{2}$ & Catalyst & & 256.9 & 0.02 & 0.0001 & 0.026 & \\
\hline & 1 & Raw material & & 84.12 & 1 & 0.005 & 0.42 & \\
\hline & $\mathrm{PhSH}$ & Raw material & & 110.17 & 1 & 0.005 & 0.55 & \\
\hline \multirow{3}{*}{ 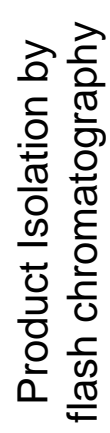 } & $\begin{array}{l}\text { Silica } \\
\text { gel }\end{array}$ & Workup & & & & & 58.2 & \\
\hline & Eluent & Solvent & & & & & $326^{[b]}$ & \\
\hline & & Product & $2 a$ & 194.29 & & & & 0.76 \\
\hline
\end{tabular}

[a] The mass of silica gel is 60 times the mass of sample. ${ }^{\left[{ }^{b}\right.}$ Amount of the eluent was taken based on Table 1. Average weight of the eluent $(\mathrm{V}$ (petroleum ether) $/ \mathrm{V}$ (ethyl acetate) $=1: 1$ ) was used for $420 \mathrm{ml}$.

$$
\begin{gathered}
E F=\frac{0.026+0.42+0.55+58.2+326 * 0.1-0.76}{0.76}=119.8 \\
c E F=\frac{0.026+0.42+0.55+58.2+326-0.76}{0.76}=505.8 \\
\text { SEF reaction }=\frac{0.026+0.421+0.551-0.760}{0.760}=0.3
\end{gathered}
$$

\section{3. $\quad[c a t]=\operatorname{Pd}(\mathrm{OAc})_{2}$ (flash chromatography $)^{[5]}$}

The calculations were made according to the published procedure [5]: " $\mathrm{Pd}(\mathrm{OAc})_{2}$ $(0.02 \mathrm{mmol}, 0.0045 \mathrm{~g})$, THF $(0.5 \mathrm{~mL})$ and alkyne $1(1 \mathrm{mmol}, 0.098 \mathrm{~mL})$ were added to a test tube. $\mathrm{PhSH}(1 \mathrm{mmol}, 0.102 \mathrm{~mL})$ was added to the mixture; the test-tube was flushed with argon and closed. The reaction was carried out under stirring at $40^{\circ} \mathrm{C}$ for $16 \mathrm{~h}$. The catalyst was filtered off from the solution through Celite, and the solvent was evaporated. The pure product $\mathbf{2 a}$ was isolated in $85 \%$ yield by flash chromatography." 
Table S3. Material Input-Output for the $\mathrm{Pd}(\mathrm{OAc})_{2}$-Catalyzed Hydrothiolation Product Purification by Flash Chromatography.

\begin{tabular}{|c|c|c|c|c|c|c|c|c|}
\hline Step & $\begin{array}{c}\text { Input } \\
\text { material }\end{array}$ & Input type & $\begin{array}{l}\text { Output } \\
\text { Material }\end{array}$ & $\begin{array}{c}\mathrm{Mw} \\
(\mathrm{g} / \mathrm{mol})\end{array}$ & Equiv. & $\begin{array}{l}\text { Input } \\
\text { (mol) }\end{array}$ & $\begin{array}{c}\text { Input } \\
\text { Weight }\end{array}$ & $\begin{array}{l}\text { Output } \\
\text { Weight }\end{array}$ \\
\hline \multirow{4}{*}{ 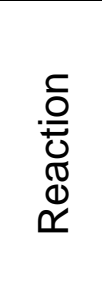 } & 1 & Raw material & & 84.12 & 1 & 0.001 & 0.084 & \\
\hline & $\mathrm{PhSH}$ & Raw material & & 110.17 & 1 & 0.001 & 0.110 & \\
\hline & $\mathrm{Pd}(\mathrm{OAc})_{2}$ & Catalyst & & 224.5 & 0.02 & 0.00002 & 0.0045 & \\
\hline & THF & Solvent & & & & & 0.445 & \\
\hline \multirow{5}{*}{ 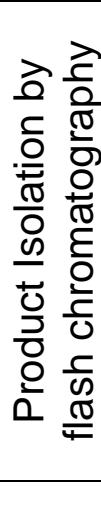 } & Celite & $\begin{array}{l}\text { Workup } \\
\text { (filtration) }\end{array}$ & & & & & $0.1^{[\mathrm{a}]}$ & \\
\hline & THF & $\begin{array}{c}\text { Solvent } \\
\text { (filtration) }\end{array}$ & & & & & 0.445 & \\
\hline & $\begin{array}{l}\text { Silica } \\
\text { gel }^{[b]}\end{array}$ & Workup & & & & & 11.64 & \\
\hline & Eluent $^{[\mathrm{c}]}$ & Solvent & & & & & 101 & \\
\hline & & Product & $2 a$ & 194.29 & & & & 0.1651 \\
\hline
\end{tabular}

[a] Amount of silica gel was taken based on author's experience of filtration of $0.5 \mathrm{~mL}$ solution trough silica gel loaded into glass Pasteur pepette. ${ }^{[b]} \mathrm{m}\left(\mathrm{SiO}_{2}\right)=60 \times \mathrm{m}_{\text {mixture. }}{ }^{[6] 7][c]}$ Amount of the eluent was taken based on Table 1. Average weight of the eluent ( $\mathrm{V}$ (petroleum ether) $/ \mathrm{V}$ (ethyl acetate) $=1: 1$ ) was used for $131 \mathrm{ml}$.

$$
\begin{gathered}
E F=\frac{0.084+0.110+0.0045+0.445 \times 0.1}{0.165}+ \\
+\frac{0.1+0.445 \times 0.1+11.64+101 \times 0.1-0.1651}{0.165}=133.0 \\
c E F=\frac{0.084+0.110+0.0045+0.445+0.1+0.445+11.64+101-0.1651}{0.165} \\
=688.5 \\
\text { sEF reaction }=\frac{0.084+0.110+0.005-0.165}{0.165}=0.2
\end{gathered}
$$




\section{4. $\quad[$ cat $]=\operatorname{Pd}(\text { hfpd })_{2}$ (flash chromatography)}

The utilized catalyst cannot be obtained from commercial sours, thus calculation of environmental factors includes steps of ligand synthesis (Scheme S2).

Step 1<smiles>CC(=O)OC(C)=O</smiles><smiles>CC(=O)CC(=O)C(F)(F)F</smiles>

Scheme S2. Preparation of $\mathrm{Pd}(\mathrm{hfpd})_{2}$.

Step 3
Step 2<smiles>CC(=O)C(F)(F)F</smiles><smiles>CCOC(=O)C(C)(C)C(=O)OCC</smiles><smiles>O=C(CC(=O)C(F)(F)F)C(F)(F)F</smiles>

2. $\mathrm{H}_{2} \mathrm{SO}_{4}$

\subsubsection{E factor evaluation for $\mathrm{Pd}(\mathrm{hfpd})_{2}$ synthesis}

Table S4. Material Input-Output for the Trifluoroacetone $\mathrm{CH}_{3} \mathrm{COCF}_{3}$ Synthesis (Step 1, Scheme S2). ${ }^{[8]}$

\begin{tabular}{|c|c|c|c|c|c|c|c|}
\hline Step & Input material & $\begin{array}{l}\text { Input } \\
\text { type }\end{array}$ & Output Material & $\begin{array}{c}\mathrm{Mw} \\
(\mathrm{g} / \mathrm{mol})\end{array}$ & $\begin{array}{l}\text { Input } \\
\text { (mol) }\end{array}$ & $\begin{array}{c}\text { Input } \\
\text { Weight }\end{array}$ & $\begin{array}{l}\text { Output } \\
\text { Weight }\end{array}$ \\
\hline \multirow{3}{*}{ 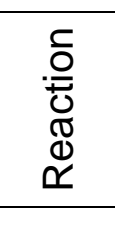 } & $\begin{array}{c}\text { Trifluoroacetic } \\
\text { acid }\end{array}$ & $\begin{array}{l}\text { Raw } \\
\text { material }\end{array}$ & & 114.03 & 0.00035 & 0.04 & \\
\hline & $\begin{array}{c}\text { Acetic } \\
\text { anhydride }\end{array}$ & $\begin{array}{l}\text { Raw } \\
\text { material }\end{array}$ & & 102.09 & 0.0021 & 0.214 & \\
\hline & & Product & Trifluoroacetone & 112.05 & & & 0.027 \\
\hline
\end{tabular}

$$
\begin{gathered}
E F=\frac{0.04+0.214-0.027}{0.027}=8.4 \\
c E F=\frac{0.04+0.214-0.027}{0.027}=8.4 \\
s E F=\frac{0.04+0.214-0.027}{0.027}=8.4
\end{gathered}
$$


Table S5. Material Input-Output for the Hexafluoroacetyl Acetone (hfpdH) Synthesis (Step 2, Scheme S2). ${ }^{[9]}$

\begin{tabular}{|c|c|c|c|c|c|c|c|c|c|}
\hline Step & $\begin{array}{c}\text { Input } \\
\text { material }\end{array}$ & $\begin{array}{l}\text { Input } \\
\text { type }\end{array}$ & $\begin{array}{c}\text { Output } \\
\text { Material }\end{array}$ & $\begin{array}{c}\mathrm{Mw} \\
(\mathrm{g} / \mathrm{mol})\end{array}$ & $\begin{array}{c}\mathrm{D}, \\
\mathrm{g} / \mathrm{mL}\end{array}$ & Equiv. & $\begin{array}{l}\text { Input } \\
\text { (mol) }\end{array}$ & $\begin{array}{c}\text { Input } \\
\text { Weight }\end{array}$ & $\begin{array}{l}\text { Output } \\
\text { Weight }\end{array}$ \\
\hline \multirow{5}{*}{ 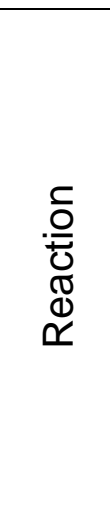 } & $\begin{array}{c}\begin{array}{c}\text { Trifluoroa } \\
\text { cetone }\end{array} \\
\end{array}$ & $\begin{array}{c}\text { Raw } \\
\text { material }\end{array}$ & & 112.05 & & 1 & 1.78 & 200 & \\
\hline & $\begin{array}{c}\text { Ethyl } \\
\text { trifluoroac } \\
\text { etate }\end{array}$ & $\begin{array}{c}\text { Raw } \\
\text { material }\end{array}$ & & 142.08 & & 1.1 & 1.97 & 280 & \\
\hline & EtONa & $\begin{array}{c}\text { Raw } \\
\text { material }\end{array}$ & & 68.05 & & 1 & 1.79 & 122 & \\
\hline & $\begin{array}{l}\mathrm{H}_{2} \mathrm{SO}_{4} \\
(98 \%)\end{array}$ & $\begin{array}{c}\text { Raw } \\
\text { material }\end{array}$ & & 98.08 & & 4.4 & 4.38 & 438 & \\
\hline & Toluene & Solvent & & 92.14 & 0.876 & & & 215 & \\
\hline ᄃ & & Product & $\begin{array}{l}\text { Hexafluoro } \\
\text { acetylacet } \\
\text { one }\end{array}$ & 208.06 & & & & & 316 \\
\hline
\end{tabular}

$$
\begin{gathered}
E F=\frac{200+280+122+438+215 * 0.1-316}{316}=2.4 \\
c E F=\frac{200+280+122+438+215-316}{316}=3.0 \\
S E F=\frac{200+280+122+438-316}{316}=2.3
\end{gathered}
$$

The procedure for $\mathrm{Pd}(\mathrm{hfpd})_{2}$ synthesis (Step 3, Scheme S2) was proposed according to the published in [10] recommendations.

A solution of $\mathrm{KOH}(0.2 \mathrm{~g}, 3.6 \mathrm{mmol})$ in water $(0.6 \mathrm{~mL})$ was prepared and $\mathrm{hfpdH}(0.81$ $\mathrm{g}, 3.9 \mathrm{mmol}$ ) was added. The resulting mixture of $\mathrm{hfpdH}$ with $\mathrm{KOH}$ in water was added to a suspension of $\mathrm{PdCl}_{2}(0.13 \mathrm{~g}, 0.73 \mathrm{mmol})$ in water $(0.25 \mathrm{~mL})$ with continuous stirring. The reaction mixture was heated to $50^{\circ} \mathrm{C}$ and $0.35 \mathrm{ml}$ of $\mathrm{H}_{2} \mathrm{O}$ was added. A precipitate of a fine mustard-yellow color was observed. The precipitate was filtered and washed with hot water (3 mL). Solid was dissolved in benzene $(0.5 \mathrm{~mL})$ and left overnight. The solution was gently decanted and $\mathrm{Pd}(\mathrm{hfpd})_{2}$ was dried under vacuum to obtain in $70 \%$ yield $(0.27 \mathrm{~g})$. 
Table S6. Material Input-Output for the Palladium (II) Hexafluoroacetylacetonate $\mathrm{Pd}(\mathrm{hfpd})_{2}$ Synthesis.

\begin{tabular}{|c|c|c|c|c|c|c|c|c|}
\hline Step & $\begin{array}{c}\text { Input } \\
\text { material }\end{array}$ & $\begin{array}{l}\text { Input } \\
\text { type }\end{array}$ & $\begin{array}{c}\text { Output } \\
\text { Material }\end{array}$ & $\begin{array}{c}\mathrm{Mw} \\
(\mathrm{g} / \mathrm{mol})\end{array}$ & $\begin{array}{c}\mathrm{D}, \\
\mathrm{g} / \mathrm{mL}\end{array}$ & $\begin{array}{l}\text { Input } \\
\text { (mol) }\end{array}$ & $\begin{array}{c}\text { Input } \\
\text { Weight }\end{array}$ & $\begin{array}{l}\text { Output } \\
\text { Weight }\end{array}$ \\
\hline \multirow{4}{*}{ 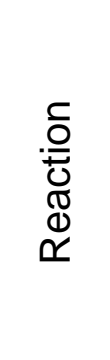 } & $\mathrm{PdCl}_{2}$ & $\begin{array}{c}\text { Raw } \\
\text { material }\end{array}$ & & 177.33 & & 0.00073 & 0.13 & \\
\hline & hfpdH & $\begin{array}{c}\text { Raw } \\
\text { material }\end{array}$ & & 208.06 & 1.49 & 0.0039 & 0.81 & \\
\hline & $\mathrm{KOH}$ & $\begin{array}{c}\text { Raw } \\
\text { material }\end{array}$ & & 56.11 & & 0.0036 & 0.2 & \\
\hline & $\mathrm{H}_{2} \mathrm{O}$ & Solvent & & & 1 & & 1.2 & \\
\hline \multirow{3}{*}{ 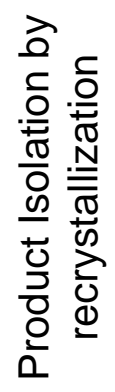 } & $\mathrm{H}_{2} \mathrm{O}$ & Solvent & & & 1 & & 3 & \\
\hline & Benzene & Solvent & & & 0.88 & & 0.57 & \\
\hline & & Product & $\mathrm{Pd}(\mathrm{hfpd})_{2}$ & 520.52 & & & & 0.27 \\
\hline
\end{tabular}

$$
\begin{gathered}
E F=\frac{0.13+0.81+0.2+0.57 * 0.1-0.27}{0.27}=3.4 \\
c E F=\frac{0.13+0.81+0.2+1.2+3+0.57-0.27}{0.27}=20.9 \\
s E F=\frac{0.13+0.81+0.2-0.27}{0.27}=3.2 \\
G W P P_{C C l}=\frac{106.4 * 0.00073 * 3880}{0.27}=1116.2^{g C O_{2}} \mathrm{gPd}(\text { hfpd })_{2}
\end{gathered}
$$

Table S7. sEF and cEF contribution to the synthesis of $\mathrm{Pd}(\mathrm{hfpd})_{2}$.

\begin{tabular}{|c|l|l|l|l|l|l|l|}
\hline $\begin{array}{l}\text { Step } \\
\#\end{array}$ & Product & $\begin{array}{l}\text { Output } \\
\text { weight }\end{array}$ & $\begin{array}{l}\text { Step } \\
\text { cEF }\end{array}$ & $\begin{array}{l}\text { cEF } \\
\text { contribution } \\
\text { to the } \\
\text { process }\end{array}$ & $\begin{array}{l}\text { Step } \\
\text { sEF }\end{array}$ & $\begin{array}{l}\text { sEF } \\
\text { contributi } \\
\text { on to the } \\
\text { process }\end{array}$ & References \\
\hline 1 & $\begin{array}{l}\text { Trifluoroac } \\
\text { etone }\end{array}$ & 0.51 & 8.4 & 4.3 & 8.4 & 4.3 & Table S4 \\
\hline 2 & hfpdH & 0.81 & 3.0 & 2.4 & 2.3 & 1.9 & Table S5 \\
\hline 3 & $\mathrm{Pd}(\mathrm{hfpd})_{2}$ & 0.27 & 20.9 & 5.6 & 3.2 & 0.9 & Table S6 \\
\hline Total & & 0.27 & & 12.3 & & 7.1 & \\
\hline
\end{tabular}




$$
\begin{gathered}
\mathrm{cEF}=12.3 / 0.27=45.3 \\
\mathrm{sEF}=7.1 / 0.27=26.3
\end{gathered}
$$

\subsubsection{E factor evaluation for vinyl sulfide $2 a$ synthesis catalyzed by $\operatorname{Pd}(\mathrm{hfpd})_{2}{ }^{[10]}$}

The calculations were made according to the published procedure [10]: "Alkyne 1 (1 mmol, $0.098 \mathrm{~mL}$ ) was added to $1 \mathrm{~mL}$ of $10^{-3} \mathrm{M} \mathrm{Pd}(\mathrm{hfpd})_{2}$ solution in toluene. To activate the catalyst, the mixture was stirred at $90{ }^{\circ} \mathrm{C}$ for $1 \mathrm{~h}$. After that, $\gamma$-terpinene $(1 \mathrm{mmol}, 0.136 \mathrm{~g})$ and $\mathrm{PhSH}(1 \mathrm{mmol}, 0.103 \mathrm{~mL}$ ) were added to the mixture. The test tube was flushed with argon and closed. The reaction was carried out at $140{ }^{\circ} \mathrm{C}$ for $24 \mathrm{~h}$.

The pure product $2 \mathrm{a}$ was obtained by flash chromatography on silica gel with gradient elution from petroleum ether to ethyl acetate."

Table S8. Material Input-Output for the $\mathrm{Pd}(\mathrm{hfpd})_{2}$-Catalyzed Hydrothiolation. Product

\begin{tabular}{|c|c|c|c|c|c|c|c|c|}
\hline Step & $\begin{array}{c}\text { Input } \\
\text { material }\end{array}$ & Input type & $\begin{array}{l}\text { Output } \\
\text { Material }\end{array}$ & $\begin{array}{c}\mathrm{Mw} \\
(\mathrm{g} / \mathrm{mol})\end{array}$ & Equiv. & $\begin{array}{l}\text { Input } \\
\text { (mol) }\end{array}$ & $\begin{array}{c}\text { Input } \\
\text { Weight }\end{array}$ & $\begin{array}{l}\text { Output } \\
\text { Weight }\end{array}$ \\
\hline \multirow{6}{*}{ 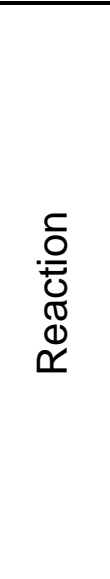 } & $\mathrm{Pd}(\mathrm{hfpd})_{2}$ & Catalyst & & 522.54 & 0.001 & 0.000001 & 0.0005 & \\
\hline & Toluene & Solvent & & & & & 0.867 & \\
\hline & $\begin{array}{c}\gamma- \\
\text { terpinene }\end{array}$ & $\begin{array}{l}\text { Raw } \\
\text { material }\end{array}$ & & 136.23 & 1 & 0.001 & 0.136 & \\
\hline & 1 & $\begin{array}{c}\text { Raw } \\
\text { material }\end{array}$ & & 84.12 & 1 & 0.001 & 0.084 & \\
\hline & $\mathrm{PhSH}$ & $\begin{array}{c}\text { Raw } \\
\text { material }\end{array}$ & & 110.17 & 1 & 0.001 & 0.110 & \\
\hline & & $\begin{array}{l}\text { Crude } \\
\text { product }\end{array}$ & $2 a$ & 194.29 & & & & 0.181 \\
\hline \multirow{3}{*}{ 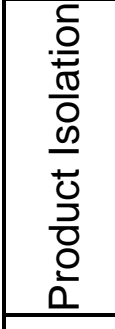 } & $2 a$ & $\begin{array}{l}\text { Crude } \\
\text { product }\end{array}$ & & 194.29 & & $9.3^{*} 10^{-4}$ & 0.181 & \\
\hline & $\begin{array}{l}\text { Silica } \\
\text { gel }^{[a]}\end{array}$ & Workup & & & & & 19.8 & \\
\hline & Eluent $^{[\mathrm{b}]}$ & Solvent & & & & & 137 & \\
\hline & & Product & $2 a$ & 194.29 & & & & 0.175 \\
\hline
\end{tabular}
Purification by Flash Chromatography.

$$
E F=\frac{0.0005+0.867 * 0.1+0.136+0.084+0.110}{0.175}+
$$




$$
\begin{gathered}
+\frac{19.8+137 * 0.1-0.175}{0.175}=192.8 \\
s E F \text { reaction }=\frac{0.0005+0.136+0.084+0.11-0.181}{0.181}=0.8 \\
c E F \text { reaction }=\frac{0.0005+0.867+0.136+0.084+0.11-0.181}{0.181}=5.6 \\
E F \text { chromatography }=\frac{0.181+19.8+137 * 0.1-0.175}{0.175}=191.5 \\
c E F \text { chromatography }=\frac{0.181+19.8+137-0.175}{0.175}=896.0
\end{gathered}
$$

Table S9. cEF and sEF contribution to the $\mathrm{Pd}(\mathrm{hfpd})_{2}$-catalyzed synthesis of $\mathbf{2 a}$ with purification by chromatography.

\begin{tabular}{|c|l|l|l|l|l|l|l|}
\hline $\begin{array}{l}\text { Step } \\
\#\end{array}$ & Product & $\begin{array}{l}\text { Output } \\
\text { weight }\end{array}$ & $\begin{array}{l}\text { Step } \\
\text { cEF }\end{array}$ & $\begin{array}{l}\text { cEF } \\
\text { contribution } \\
\text { to the } \\
\text { process }\end{array}$ & $\begin{array}{l}\text { Step } \\
\text { sEF }\end{array}$ & $\begin{array}{l}\text { sEF } \\
\text { contribution } \\
\text { to the } \\
\text { process }\end{array}$ & References \\
\hline 1 & $\mathrm{Pd}(\mathrm{hfpd})_{2}$ & 0.0005 & 45.1 & 0.02 & 24.1 & 0.01 & Table S7 \\
\hline 2 & $\mathbf{2 a}$ & 0.175 & 902 & 157.82 & 0.8 & 0.14 & Table S8 \\
\hline Total & & 0.175 & & 157.84 & & 0.15 & \\
\hline
\end{tabular}

$$
\begin{gathered}
\mathrm{cEF}=157.84 / 0.175=901.9 \\
\mathrm{sEF}=0.15 / 0.175=0.9
\end{gathered}
$$

\section{5. [cat] $=(\mathrm{IMes}) \mathrm{Pd}(\mathrm{acac}) \mathrm{Cl}$ (dry column vacuum chromatography $)^{[11]}$}

The utilized catalyst cannot be obtained from the commercial source, thus calculation of environmental factors includes ligand synthesis steps (Scheme S3). 


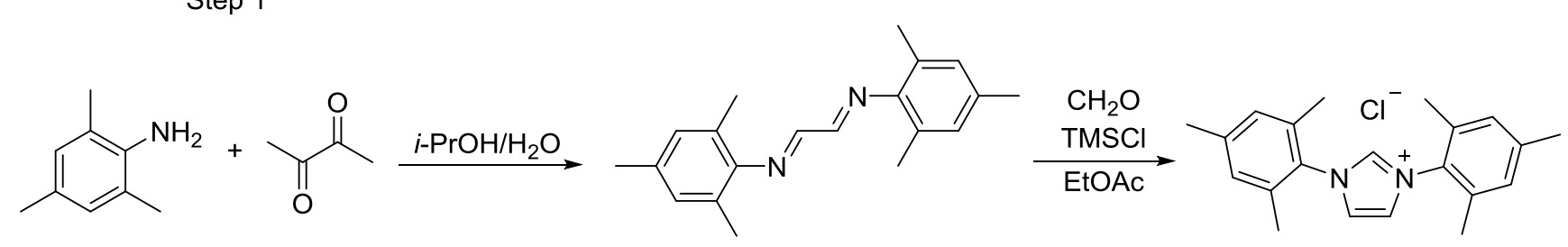

Step 3
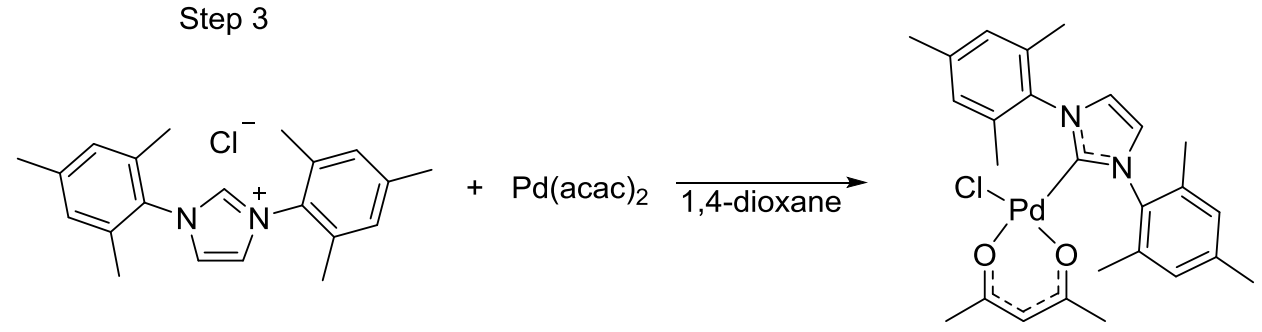

Scheme S3. Preparation of (IMes) Pd(acac)Cl.

\subsection{1. (IMes) $\mathrm{Pd}(\mathrm{acac}) \mathrm{Cl}$ Synthesis}

E factor evaluation for dimesityl ethylenediimine synthesis (Step 1, Scheme S3). ${ }^{[12]}$

The calculations were made according to the published procedure [12]: "Prepare a solution of glyoxal $\left(9 \mathrm{~mL}, 79 \mathrm{mmol}, 40 \%\right.$ wt in $\mathrm{H}_{2} \mathrm{O}$ ) in isopropanol $(100 \mathrm{~mL})$ and water $(200$ $\mathrm{mL}$ ) at $0{ }^{\circ} \mathrm{C}$. Add mesitylamine (25 mL, 2.2 equiv.); a yellow precipitate forms immediately. Stir rapidly for 24 hours at room temperature. Collect yellow precipitate on a coarse frit, and wash water $(1 \times 100 \mathrm{~mL})$ and hexanes $(3 \times 100 \mathrm{~mL})$. Add $50 \mathrm{~mL}$ acetone to the wet solid and pump down the resulting suspension. Dry the solid under vacuum for one day to obtain the diimine as yellow solid in $89 \%$ yield $(20.6 \mathrm{~g})$." 
Table S10. Material Input-Output for the Dimesityl Ethylenediimine $[\mathrm{CH}(=\mathrm{NMes})]_{2}$ Synthesis.

\begin{tabular}{|c|c|c|c|c|c|c|c|c|}
\hline Step & $\begin{array}{c}\text { Input } \\
\text { material }\end{array}$ & $\begin{array}{l}\text { Input } \\
\text { type }\end{array}$ & $\begin{array}{c}\text { Output } \\
\text { Material }\end{array}$ & $\begin{array}{c}\mathrm{Mw} \\
(\mathrm{g} / \mathrm{mol})\end{array}$ & $\begin{array}{c}\mathrm{D} \\
(\mathrm{g} / \mathrm{mL})\end{array}$ & $\begin{array}{l}\text { Input } \\
\text { (mol) }\end{array}$ & $\begin{array}{c}\text { Input } \\
\text { Weight }\end{array}$ & $\begin{array}{l}\text { Output } \\
\text { Weight }\end{array}$ \\
\hline \multirow{5}{*}{ 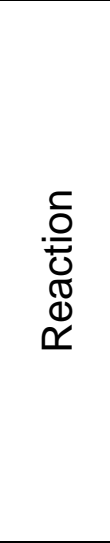 } & Glyoxal & $\begin{array}{c}\text { Raw } \\
\text { material }\end{array}$ & & 58.04 & & 0.079 & 4.59 & \\
\hline & $\begin{array}{c}\mathrm{H}_{2} \mathrm{O} \\
\text { (Glyoxal } \\
\text { solution) }\end{array}$ & Solvent & & & 1 & & 6.88 & \\
\hline & $i-\mathrm{PrOH}$ & Solvent & & & 0.79 & & 79.00 & \\
\hline & $\mathrm{H}_{2} \mathrm{O}$ & Solvent & & & 1 & & 200 & \\
\hline & $\begin{array}{l}\text { Mesityl } \\
\text { amine } \\
(98 \%)\end{array}$ & $\begin{array}{c}\text { Raw } \\
\text { material }\end{array}$ & & 135.21 & 0.96 & 0.174 & 23.99 & \\
\hline \multirow{4}{*}{ 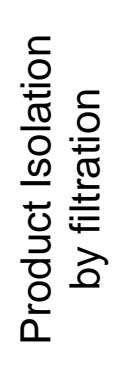 } & $\mathrm{H}_{2} \mathrm{O}$ & Solvent & & & 1 & & 100 & \\
\hline & Hexane & Solvent & & & 0.66 & & 198 & \\
\hline & Acetone & Solvent & & & 0.78 & & 39 & \\
\hline & & Product & $\begin{array}{l}\text { Dimesityl } \\
\text { ethylene } \\
\text { diimine }\end{array}$ & 292.43 & & & & 20.6 \\
\hline
\end{tabular}

$$
\begin{gathered}
E F=\frac{4.59+79.00 * 0.1+23.99+198 * 0.1+39 * 0.1-20.6}{20.6}=1.9 \\
c E F=\frac{4.59+6.88+79.00+200+23.99+100+198+39-20.6}{20.6}=30.6 \\
s E F=\frac{4.59+23.99-20.6}{20.6}=0.4
\end{gathered}
$$

\section{E factor evaluation for 1,3-Bis-(2,4,6-trimethylphenyl)imidazolium chloride} (IMes.HCl) synthesis (Step 2, Scheme S3). ${ }^{[13]}$

The calculations were made according to the published procedure [13]: "A round bottom flask containing EtOAc (technical grade, distilled in a rotatory evaporator over $\mathrm{K}_{2} \mathrm{CO}_{3}$ ) was heated to $70{ }^{\circ} \mathrm{C}$ in an oil bath. Dimesityl ethylene diimine $(2.19 \mathrm{~g}, 7.5 \mathrm{mmol})$ and paraformaldehyde $(0.23 \mathrm{~g}, 7.5 \mathrm{mmol})$ were added and the walls washed with EtOAc. A solution of $\mathrm{TMSCl}(0.81 \mathrm{~g}, 134.5 \mathrm{mmol})$ was added dropwise over $45 \mathrm{~min}$ with vigorous stirring, and the resulting yellow suspension stirred for $2 \mathrm{~h}$ at $70^{\circ} \mathrm{C}$. Total amount of EtOAc in reaction flask is about $67.5 \mathrm{~mL}$. After cooling to $10^{\circ} \mathrm{C}$ (icebath) with stirring, the suspension was filtered and the solid washed with EtOAc $20 \mathrm{~mL}$ and tBuOMe $20 \mathrm{~mL}$. The solid was dried to constant weight in an open dish in a well-ventilated oven at $100{ }^{\circ} \mathrm{C}(1 \mathrm{~d})$, giving $1.76 \mathrm{~g}$ (69\%) of IMes.HCl." 
Table S11. Material Input-Output for the IMes·HCl Synthesis.

\begin{tabular}{|c|c|c|c|c|c|c|c|c|c|}
\hline Step & $\begin{array}{c}\text { Input } \\
\text { material }\end{array}$ & $\begin{array}{l}\text { Input } \\
\text { type }\end{array}$ & $\begin{array}{l}\text { Output } \\
\text { Material }\end{array}$ & $\begin{array}{c}\mathrm{Mw} \\
(\mathrm{g} / \mathrm{mol})\end{array}$ & $\begin{array}{c}\mathrm{D}, \\
\mathrm{g} / \mathrm{mL}\end{array}$ & $\begin{array}{l}\text { Equi } \\
\text { v. }\end{array}$ & $\begin{array}{l}\text { Input } \\
\text { (mol) }\end{array}$ & $\begin{array}{c}\text { Input } \\
\text { Weigh } \\
\mathrm{t}\end{array}$ & $\begin{array}{l}\text { Out } \\
\text { put } \\
\text { Wei } \\
\text { ght }\end{array}$ \\
\hline \multirow{4}{*}{ 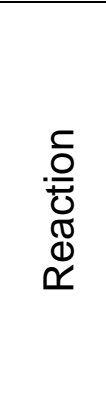 } & $\mathrm{CH}_{2} \mathrm{O}$ & $\begin{array}{c}\text { Raw } \\
\text { material }\end{array}$ & & 30.3 & & 1 & 0.0075 & 0.23 & \\
\hline & TMSCI & $\begin{array}{c}\text { Raw } \\
\text { material }\end{array}$ & & 108.64 & 0.86 & 1 & 0.0075 & 0.81 & \\
\hline & $\begin{array}{l}\text { Dimesityl } \\
\text { ethylene } \\
\text { diimine }\end{array}$ & $\begin{array}{l}\text { Raw } \\
\text { material }\end{array}$ & & 292.43 & & 1 & 0.0075 & 2.19 & \\
\hline & EtOAc & Solvent & & & 0.90 & & & 60.75 & \\
\hline \multirow{3}{*}{ 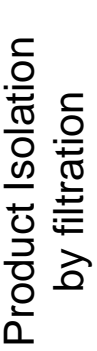 } & EtOAc & Solvent & & & 0.90 & & & $18^{[\mathrm{a}]}$ & \\
\hline & $t$-BuOMe & Solvent & & & 0.74 & & & $14.8^{[\mathrm{a}]}$ & \\
\hline & & Product & IMes $\cdot \mathrm{HCl}$ & 340.89 & & & & & 1.76 \\
\hline
\end{tabular}

$$
\begin{gathered}
E F=\frac{0.23+0.81+2.19+60.75 * 0.1+18 * 0.1+14.8 * 0.1-1.76}{1.76}=6.2 \\
c E F=\frac{0.23+0.81+2.19+60.75+18+14.8-1.76}{1.76}=54.0 \\
s E F=\frac{0.23+0.81+2.19-1.76}{1.76}=0.8
\end{gathered}
$$

\section{E factor evaluation for (IMes)Pd(acac)CI Synthesis (Step 3, Scheme S3). ${ }^{[1]}$}

The calculations were made according to the published procedure [11]: "Reaction of $\mathrm{Pd}(\mathrm{acac})_{2}\left(1.34 \times 10^{-3} \mathrm{~mol}, 0.408 \mathrm{~g}\right)$ with IMes $\cdot \mathrm{HCl}\left(1.47 \times 10^{-3} \mathrm{~mol}, 0.500 \mathrm{~g}\right)$ in 1,4-dioxane $(8 \mathrm{~mL})$ was carried out under reflux for $24 \mathrm{~h}$. The color of reaction mixture changed from yellow to brown. 1,4-dioxane was evaporated under vacuum and $\mathrm{Et}_{2} \mathrm{O}$ was added. The mixture was filtered off over the plug of Celite 545 and the solid residue was washed with $\mathrm{Et}_{2} \mathrm{O}(3 \times 5 \mathrm{~mL})$. The product was washed off from Celite with $\mathrm{DCM}(15 \mathrm{~mL})$ affording yellow solution. DCM was evaporated under vacuum and $0.55 \mathrm{~g}(75 \%)$ of the complex was obtained." 
Table S12. Material Input-Output for the (IMes) Pd(acac)Cl Synthesis.

\begin{tabular}{|c|c|c|c|c|c|c|c|c|c|}
\hline Step & $\begin{array}{c}\text { Input } \\
\text { material }\end{array}$ & $\begin{array}{l}\text { Input } \\
\text { type }\end{array}$ & $\begin{array}{l}\text { Output } \\
\text { Material }\end{array}$ & $\begin{array}{c}\mathrm{Mw} \\
(\mathrm{g} / \mathrm{mol})\end{array}$ & $\begin{array}{c}\mathrm{D}, \\
\mathrm{g} / \mathrm{mL}\end{array}$ & $\begin{array}{c}\text { Equiv } \\
.\end{array}$ & $\begin{array}{l}\text { Input } \\
\text { (mol) }\end{array}$ & $\begin{array}{c}\text { Input } \\
\text { Weig } \\
\mathrm{ht}\end{array}$ & $\begin{array}{l}\text { Output } \\
\text { Weight }\end{array}$ \\
\hline \multirow{3}{*}{$\begin{array}{l}\check{D} \\
\stackrel{0}{0} \\
\mathbb{J} \\
\mathbb{D} \\
\simeq\end{array}$} & $\mathrm{Pd}(\mathrm{acac})_{2}$ & $\begin{array}{c}\text { Raw } \\
\text { material }\end{array}$ & & 304.64 & & 1 & 0.0013 & 0.41 & \\
\hline & IMes $\cdot \mathrm{HCl}$ & $\begin{array}{c}\text { Raw } \\
\text { material }\end{array}$ & & 340.89 & 1 & 1.1 & 0.0015 & 0.5 & \\
\hline & Dioxane & Solvent & & & 1.03 & & & 8.24 & \\
\hline \multirow{4}{*}{ 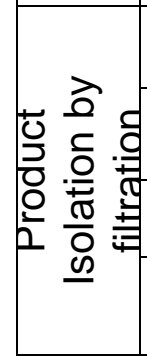 } & Celite & Workup & & & & & & $0.6^{[a]}$ & \\
\hline & $\mathrm{Et}_{2} \mathrm{O}$ & Solvent & & & 0.71 & & & $14.2^{[b]}$ & \\
\hline & DCM & Solvent & & & 1.33 & & & 19.95 & \\
\hline & & Product & $\begin{array}{c}\text { (IMes)Pd(a } \\
\text { (ac)Cl }\end{array}$ & 545.42 & & & & & 0.55 \\
\hline
\end{tabular}

$$
\begin{gathered}
E F=\frac{0.41+0.5+8.24 * 0.1+0.6+14.2 * 0.1+19.95 * 0.1-0.55}{0.55}=9.5 \\
c E F=\frac{0.41+0.5+8.24+0.6+14.2+19.95-0.55}{0.55}=78.8 \\
\mathrm{sEF}=\frac{0.41+0.5+0.6-0.55}{0.55}=1.7 \\
G W P \text { Pd(acac })_{2}=\frac{106.4 * 0.0013 * 3880}{0.55}=975.8 \frac{\mathrm{g} \mathrm{CO}_{2}}{\mathrm{~g} \text { IMes Pd acac } \mathrm{Cl}}
\end{gathered}
$$

Table S13. sEF and cEF contribution to the synthesis of (IMes) $\mathrm{Pd}(\mathrm{acac}) \mathrm{Cl}$.

\begin{tabular}{|c|l|l|l|l|l|l|l|}
\hline $\begin{array}{l}\text { Step } \\
\#\end{array}$ & Product & $\begin{array}{l}\text { Output } \\
\text { weight }\end{array}$ & $\begin{array}{l}\text { Step } \\
\text { cEF }\end{array}$ & $\begin{array}{l}\text { cEF } \\
\text { contribution } \\
\text { to the } \\
\text { process }\end{array}$ & $\begin{array}{l}\text { Step } \\
\text { sEF }\end{array}$ & $\begin{array}{l}\text { sEF } \\
\text { contribution } \\
\text { to the } \\
\text { process }\end{array}$ & References \\
\hline 1 & $\begin{array}{l}\text { Dimesitul } \\
\text { ethylenediimine }\end{array}$ & 0.62 & 30.6 & 18.97 & 0.4 & 0.25 & Table S10 \\
\hline 2 & IMes $\cdot \mathrm{HCl}$ & 0.5 & 54.0 & 27.00 & 0.8 & 0.4 & Table S11 \\
\hline 3 & IMesPd(acac)Cl & 0.55 & 78.8 & 43.34 & 2 & 1.1 & Table S12 \\
\hline Total & & 0.55 & & 89.31 & & 1.75 & \\
\hline
\end{tabular}




$$
\begin{gathered}
\mathrm{cEF}=89.31 / 0.55=162.4 \\
\mathrm{sEF}=1.75 / 0.55=3.2
\end{gathered}
$$

\subsubsection{E factor evaluation for synthesis of vinyl sulfide $2 a$ catalyzed be (IMes)Pd(acac)Cl}

The calculations were made according to the published procedure [11]: "(IMes) $\mathrm{Pd}(\mathrm{acac}) \mathrm{Cl}(0.005 \mathrm{mmol}, 0.0027 \mathrm{~g}), \mathrm{Et}_{3} \mathrm{~N}(0.02 \mathrm{mmol}, 0.0028 \mathrm{~mL}), \gamma$-terpinene $(0.5$ mmol, $0.080 \mathrm{~mL})$ and alkyne $1(0.5 \mathrm{mmol}, 0.049 \mathrm{~mL})$ were mixed in a screw-capped test tube. $\mathrm{PhSH}(0.5 \mathrm{mmol}, 0.051 \mathrm{~mL})$ was subsequently added; the reaction vessel was flushed with argon and closed. The reaction was carried out for $8 \mathrm{~h}$ at $100^{\circ} \mathrm{C}$.

The catalyst was filtered off from the solution through Celite with $5 \mathrm{~mL}$ of petroleum ether. The pure product $2 \mathbf{a}$ was isolated in $91 \%$ yield by dry column vacuum chromatography with petroleum ether/EtOAc gradient elution."

Table S14. Material Input-Output for the (IMes)Pd(acac)Cl-catalyzed Hydrothiolation. Product

\begin{tabular}{|c|c|c|c|c|c|c|c|c|}
\hline Step & $\begin{array}{l}\text { Input } \\
\text { material }\end{array}$ & Input type & $\begin{array}{c}\text { Output } \\
\text { Material }\end{array}$ & $\begin{array}{c}\mathrm{Mw} \\
(\mathrm{g} / \mathrm{mol})\end{array}$ & $\begin{array}{c}\text { Equiv } \\
\text {. }\end{array}$ & $\begin{array}{l}\text { Input } \\
\text { (mol) }\end{array}$ & $\begin{array}{c}\text { Input } \\
\text { Weight }\end{array}$ & $\begin{array}{l}\text { Output } \\
\text { Weight }\end{array}$ \\
\hline \multirow{6}{*}{ 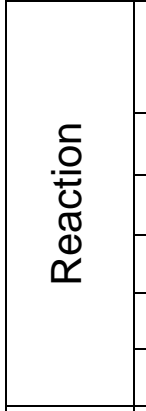 } & $\begin{array}{c}\text { (IMes) } \mathrm{Pd}(\mathrm{a} \\
\text { (ac)Cl }\end{array}$ & Catalyst & & 546.42 & 0.01 & $0.5 \times 10^{-5}$ & 0.0027 & \\
\hline & $\mathrm{Et}_{3} \mathrm{~N}$ & Raw material & & 101.19 & 0.04 & $2 \times 10^{-5}$ & 0.002 & \\
\hline & $\gamma$-terpinene & Raw material & & 136.23 & 1 & 0.0005 & 0.068 & \\
\hline & 1 & Raw material & & 84.12 & 1 & 0.0005 & 0.042 & \\
\hline & PhSH & Raw material & & 110.17 & 1 & 0.0005 & 0.055 & \\
\hline & & Crude Product & $2 a$ & 194.29 & & & & 0.091 \\
\hline \multirow{5}{*}{ 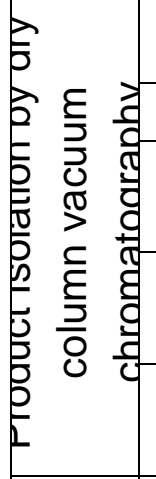 } & $2 a$ & Crude Product & & 194.29 & & & 0.091 & \\
\hline & Celite & Workup (filtration) & & & & & $0.1^{[a]}$ & \\
\hline & $\begin{array}{l}\text { Petroleum } \\
\text { ether }\end{array}$ & Solvent (filtration) & & & & & 3.5 & \\
\hline & Silica gel ${ }^{[b]}$ & $\begin{array}{c}\text { Workup } \\
\text { (chromatography) }\end{array}$ & & & & & $15^{[b]}$ & \\
\hline & Eluent $^{[\mathrm{b}]}$ & $\begin{array}{c}\text { Solvent } \\
\text { (chromatography) }\end{array}$ & & & & & 128 & \\
\hline & & Product & $2 a$ & 194.29 & & & & 0.088 \\
\hline
\end{tabular}
Purification by Dry Column Vacuum Chromatography. 


$$
\begin{aligned}
& E F=\frac{0.0027+0.002+0.068+0.042+0.055}{0.088}+ \\
& +\frac{0.1+3.5 * 0.1+15+128 * 0.1-0.088}{0.088}=322.0 \\
& c E F=\frac{0.0027+0.002+0.068+0.042+0.055}{0.088}+ \\
& +\frac{0.1+3.5+15+128-0.088}{0.088}=1666.8
\end{aligned}
$$

$E F$ chromatography $=\frac{0.091+0.1+3.5 * 0.1+15+128 * 0.1-0.088}{0.088}=321.1$

$$
c E F \text { chromatography }=\frac{0.091+0.1+3.5+15+128-0.088}{0.088}=1665.9
$$

Table S15. cEF and sEF contribution to the (IMes)Pd(acac)Cl-catalyzed synthesis of $\mathbf{2 a}$ with purification by chromatography.

\begin{tabular}{|c|l|l|l|l|l|l|l|}
\hline $\begin{array}{l}\text { Step } \\
\#\end{array}$ & Product & $\begin{array}{l}\text { Output } \\
\text { weight }\end{array}$ & $\begin{array}{l}\text { Step } \\
\text { cEF }\end{array}$ & $\begin{array}{l}\text { cEF } \\
\text { contributio } \\
\mathbf{n} \text { to the } \\
\text { process }\end{array}$ & $\begin{array}{l}\mathbf{p} \\
\mathbf{s E F}\end{array}$ & $\begin{array}{l}\text { sEF } \\
\text { contributio } \\
\mathbf{n} \text { to the } \\
\text { process }\end{array}$ & \begin{tabular}{l} 
Reference \\
\hline 1
\end{tabular} \\
$\begin{array}{l}\text { (IMes) } \mathrm{Pd}(\mathrm{aca} \\
\mathrm{C}) \mathrm{Cl}\end{array}$ & 0.0027 & 162.4 & 0.44 & 3.2 & 0.02 & Table S13 \\
\hline 2 & $\mathbf{2 a}$ & 0.088 & 1666.8 & 146.7 & 0.8 & 0.07 & Table S14 \\
\hline $\begin{array}{l}\text { Tota } \\
\text { I }\end{array}$ & & 0.088 & & 147.14 & & 0.08 & \\
\hline
\end{tabular}

$$
\begin{gathered}
\mathrm{cEF}=147.14 / 0.088=1672.0 \\
\mathrm{sEF}=0.08 / 0.088=0.9
\end{gathered}
$$




\section{EF and cEF values in the synthesis of $2 a$, purified by distillation}

\section{1. $\quad[$ cat $]=\mathrm{Ni}(\mathrm{acac})_{2}$ (distillation)}

Table S16. Material Input-Output for the $\mathrm{Ni}(\mathrm{acac})_{2}$-Catalyzed Hydrothiolation. Product Purification by Distillation.

\begin{tabular}{|c|c|c|c|c|c|c|c|c|}
\hline Step & $\begin{array}{l}\text { Input } \\
\text { material }\end{array}$ & $\begin{array}{l}\text { Input } \\
\text { type }\end{array}$ & $\begin{array}{l}\text { Output } \\
\text { Material }\end{array}$ & $\underset{(\mathrm{g} / \mathrm{mol})}{\mathrm{Mw}}$ & Equiv. & $\begin{array}{l}\text { Input } \\
\text { (mol) }\end{array}$ & $\begin{array}{c}\text { Input } \\
\text { Weight }\end{array}$ & $\begin{array}{l}\text { Output } \\
\text { Weight }\end{array}$ \\
\hline \multirow{3}{*}{ 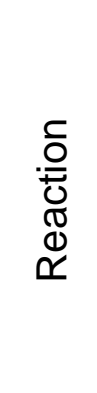 } & $\mathrm{Ni}(\mathrm{acac})_{2}$ & Catalyst & & 256.9 & 0.02 & 0.0003 & 0.077 & \\
\hline & 1 & $\begin{array}{c}\text { Raw } \\
\text { material }\end{array}$ & & 84.12 & 1 & 0.015 & 1.262 & \\
\hline & $\mathrm{PhSH}$ & $\begin{array}{c}\text { Raw } \\
\text { material }\end{array}$ & & 110.18 & 2 & 0.03 & 3.305 & \\
\hline \multirow{2}{*}{ 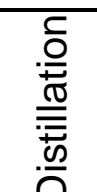 } & Acetone $^{[a]}$ & Solvent & & & & & 0.458 & \\
\hline & & Product & $2 a$ & 194.29 & & & & 1.253 \\
\hline
\end{tabular}

$$
\begin{gathered}
E F=\frac{0.077+1.262+3.305+0.458 * 0.1-1.253}{1.253}=2.7 \\
c E F=\frac{0.077+1.262+3.305+0.458-1.253}{1.253}=3.1 \\
s E F \text { reaction }=\frac{0.077+1.262+3.305-1.253}{1.253}=2.7 \\
G W P \text { Ni acac }_{2}=\frac{58.79 * 0.0003 * 6.5}{1.253}=0.1^{g_{C O}} \mathrm{~g} \mathbf{2 a}
\end{gathered}
$$




\section{2. $\quad[$ cat $]=\operatorname{Pd}(\mathrm{OAc})_{2}$ (distillation)}

Table S17. Material Input-Output for the $\mathrm{Pd}(\mathrm{OAc})_{2}$-Catalyzed Hydrothiolation with Product Purification by Distillation.

\begin{tabular}{|c|c|c|c|c|c|c|c|c|}
\hline Step & $\begin{array}{c}\text { Input } \\
\text { material }\end{array}$ & $\begin{array}{l}\text { Input } \\
\text { type }\end{array}$ & $\begin{array}{l}\text { Output } \\
\text { Material }\end{array}$ & $\begin{array}{c}\text { Mw } \\
(\mathrm{g} / \mathrm{mol})\end{array}$ & Equiv. & $\begin{array}{l}\text { Input } \\
\text { (mol) }\end{array}$ & $\begin{array}{l}\text { Input } \\
\text { Weight }\end{array}$ & $\begin{array}{l}\text { Output } \\
\text { Weight }\end{array}$ \\
\hline \multirow{3}{*}{ 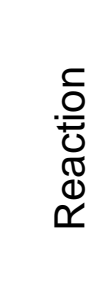 } & 1 & $\begin{array}{c}\text { Raw } \\
\text { material }\end{array}$ & & 84.12 & 1 & 0.0150 & 1.262 & \\
\hline & $\mathrm{PhSH}$ & $\begin{array}{c}\text { Raw } \\
\text { material }\end{array}$ & & 110.18 & 1 & 0.0150 & 1.653 & \\
\hline & $\mathrm{Pd}(\mathrm{OAc})_{2}$ & Catalyst & & 224.5 & 0.02 & 0.0003 & 0.067 & \\
\hline \multirow[t]{2}{*}{ 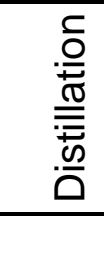 } & Acetone $e^{[a]}$ & & & & & & 0.458 & \\
\hline & & Product & $2 a$ & 194.29 & & & & 1.923 \\
\hline
\end{tabular}

$$
\begin{gathered}
E F=\frac{0.067+1.262+1.653+0.458 * 0.1-1.923}{1.923}=0.6 \\
c E F=\frac{0.067+1.262+1.653+0.458-1.923}{1.923}=0.8 \\
\text { SEF reaction }=\frac{0.067+1.262+1.653-1.923}{1.923}=0.6 \\
G W P \text { Pd OAC }_{2}=\frac{106.4 * 0.0003 * 3880}{1.923}=64 . \mathrm{gCO}_{2} \mathrm{~g} \mathbf{2 a}
\end{gathered}
$$




\section{3. $[$ cat $]=\operatorname{Pd}(\text { hfpd })_{2}$ (distillation)}

Table S18. Material Input-Output for the $\mathrm{Pd}(\mathrm{hfpd})_{2}$-Catalyzed Hydrothiolation with Product Purification by Distillation.

\begin{tabular}{|c|c|c|c|c|c|c|c|c|}
\hline Step & $\begin{array}{l}\text { Input } \\
\text { material }\end{array}$ & $\begin{array}{l}\text { Input } \\
\text { type }\end{array}$ & $\begin{array}{l}\text { Output } \\
\text { Material }\end{array}$ & $\begin{array}{c}\mathrm{Mw} \\
(\mathrm{g} / \mathrm{mol})\end{array}$ & Equiv. & $\begin{array}{l}\text { Input } \\
\text { (mol) }\end{array}$ & $\begin{array}{c}\text { Input } \\
\text { Weight }\end{array}$ & $\begin{array}{l}\text { Output } \\
\text { Weight }\end{array}$ \\
\hline \multirow{6}{*}{ 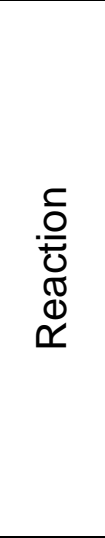 } & $\mathrm{Pd}(\mathrm{hfpd})_{2}$ & Catalyst & & 522.54 & 0.001 & 0.000015 & 0.008 & \\
\hline & $\gamma$-terpinene & $\begin{array}{c}\text { Raw } \\
\text { material }\end{array}$ & & 136.23 & 1 & 0.015 & 2.043 & \\
\hline & 1 & $\begin{array}{c}\text { Raw } \\
\text { material }\end{array}$ & & 84.12 & 1 & 0.015 & 1.262 & \\
\hline & $\mathrm{PhSH}$ & $\begin{array}{c}\text { Raw } \\
\text { material }\end{array}$ & & 110.17 & 1 & 0.015 & 1.653 & \\
\hline & Toluene & Solvent & & & & & 0.867 & \\
\hline & & $\begin{array}{c}\text { Crude } \\
\text { product }\end{array}$ & $2 a$ & 194.29 & & & & 2.710 \\
\hline \multirow{3}{*}{ 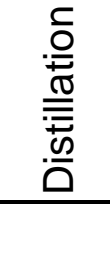 } & $2 a$ & $\begin{array}{c}\text { Crude } \\
\text { product }\end{array}$ & & 194.29 & & & 2.710 & \\
\hline & Acetone $^{[\mathrm{a}]}$ & Solvent & & & & & 0.458 & \\
\hline & & Product & $2 a$ & 194.29 & & & & 2.186 \\
\hline
\end{tabular}

${ }^{\text {[a] }}$ To avoid additional product loss acetone was used to wash the product from the distillation unit.

$$
\begin{gathered}
E F=\frac{0.008+2.043+1.262+1.653+0.867 * 0.1+0.458 * 0.1-2.186}{2.186}=1.3 \\
c E F=\frac{0.008+2.043+1.262+1.653+0.867+0.458-2.186}{2.186}=1.9 \\
s E F \text { reaction }=\frac{0.008+1.262+1.653+2.043-2.186}{2.186}=1.3 \\
E F \text { distillation }=\frac{2.710+0.458 * 0.1-2.186}{2.186}=0.3 \\
c E F \text { distillation }=\frac{2.710+0.458-2.186}{2.186}=0.4
\end{gathered}
$$


Table S19. cEF and SEF contribution to the $\mathrm{Pd}(\mathrm{hfpd})_{2}$-catalyzed synthesis of $\mathbf{2 a}$ with purification by distillation.

\begin{tabular}{|c|l|l|l|l|l|l|l|l|}
\hline $\begin{array}{c}\text { Step } \\
\#\end{array}$ & Product & $\begin{array}{l}\text { Output } \\
\text { weight }\end{array}$ & $\begin{array}{l}\text { Step } \\
\text { cEF }\end{array}$ & $\begin{array}{l}\text { cEF } \\
\text { contribution } \\
\text { to the } \\
\text { process }\end{array}$ & $\begin{array}{l}\text { Step } \\
\text { sEF }\end{array}$ & $\begin{array}{l}\text { sEF } \\
\text { contribution } \\
\text { to the } \\
\text { process }\end{array}$ & $\begin{array}{l}\text { GWP } \\
\text { (cat) }\end{array}$ & References \\
\hline 1 & $\mathrm{Pd}(\mathrm{hfpd})_{2}$ & 0.008 & 45.6 & 0.36 & 26.3 & 0.21 & & Table S7 \\
\hline 2 & $\mathbf{2 a}$ & 2.186 & 1.9 & 4.15 & 1.3 & 2.84 & & Table S18 \\
\hline Total & & 2.186 & & 4.51 & & 3.05 & & \\
\hline
\end{tabular}

$$
\begin{aligned}
& \mathrm{cEF}=4.51 / 2.186=2.1 \\
& \mathrm{sEF}=3.05 / 2.186=1.4
\end{aligned}
$$

According to Eq. S19 GWP(PdCl $)_{2}=1116.2 \mathrm{~g} \mathrm{CO}_{2} / \mathrm{g} \mathrm{Pd}(\mathrm{hfpd})_{2}$, thus

$$
\text { GWP Pd hfpd } 2=\frac{1116.2 * 0.008}{2.186}=4.1^{\mathrm{gCO}_{2}} \mathrm{~g} \mathrm{2a}
$$

\section{4. $\quad[\mathrm{cat}]=(\mathrm{IMes}) \mathrm{Pd}(\mathrm{acac}) \mathrm{Cl}$ (distillation)}

\begin{tabular}{|c|c|c|c|c|c|c|c|c|}
\hline $\begin{array}{c}\mathrm{S} \\
\text { te } \\
\mathrm{p}\end{array}$ & Input material & Input type & $\begin{array}{l}\text { Output } \\
\text { Material }\end{array}$ & $\begin{array}{c}\mathrm{Mw} \\
(\mathrm{g} / \mathrm{mol})\end{array}$ & $\begin{array}{l}\text { Equi } \\
\text { v. }\end{array}$ & $\begin{array}{l}\text { Input } \\
\text { (mol) }\end{array}$ & $\begin{array}{c}\text { Input } \\
\text { Weight }\end{array}$ & $\begin{array}{l}\text { Output } \\
\text { Weight }\end{array}$ \\
\hline \multirow{6}{*}{ 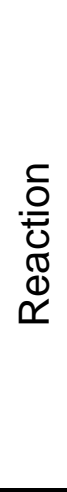 } & (IMes)Pd(acac)Cl & Catalyst & & 546.42 & 0.01 & 0.00015 & 0.082 & \\
\hline & $\mathrm{Et}_{3} \mathrm{~N}$ & Raw material & & 101.19 & 0.04 & 0.0006 & 0.061 & \\
\hline & $\gamma$-terpinene & Raw material & & 136.23 & 1 & 0.015 & 2.043 & \\
\hline & 1 & Raw material & & 84.12 & 1 & 0.015 & 1.262 & \\
\hline & $\mathrm{PhSH}$ & Raw material & & 110.17 & 1 & 0.015 & 1.653 & \\
\hline & & $\begin{array}{c}\text { Crude } \\
\text { product }\end{array}$ & $2 a$ & 194.29 & & & & 2.739 \\
\hline \multirow{3}{*}{ 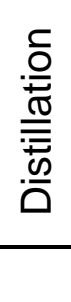 } & $2 a$ & $\begin{array}{l}\text { Crude } \\
\text { product }\end{array}$ & $2 a$ & 194.29 & & & 2.739 & \\
\hline & Acetone $^{[\mathrm{a}]}$ & Solvent & & & & & 0.458 & \\
\hline & & Product & $2 a$ & 194.29 & & & & 2.186 \\
\hline
\end{tabular}

Table S20. Material Input-Output for the (IMed)Pd(acac)Cl-Catalyzed Hydrothiolation with Product Purification by Distillation.

[a] To avoid additional product loss acetone was used to wash the product from the distillation unit.

$$
E F=\frac{0.082+0.061+2.043+1.262+1.653+0.458 * 0.1-2.186}{2.186}=1.4
$$




$$
\begin{gathered}
c E F=\frac{0.082+0.061+2.043+1.262+1.653+0.458-2.186}{2.186}=1.5 \\
s E F \text { reaction }=\frac{0.082+0.061+2.043+1.262+1.653-2.186}{2.186}=1.3 \\
E F \text { distillation }=\frac{2.739+0.458 * 0.1-2.186}{2.186}=0.3 \\
C E F \text { distillation }=\frac{2.739+0.458-2.186}{2.186}=0.5
\end{gathered}
$$

Table S21. cEF and SEF contribution to the IMesPd(acac)Cl-catalyzed synthesis of 2 a with purification by distillation.

\begin{tabular}{|c|l|l|l|l|l|l|l|}
\hline $\begin{array}{l}\text { Step } \\
\#\end{array}$ & Product & $\begin{array}{l}\text { Output } \\
\text { weight }\end{array}$ & $\begin{array}{l}\text { Step } \\
\text { cEF }\end{array}$ & $\begin{array}{l}\text { cEF } \\
\text { contribution } \\
\text { to the } \\
\text { process }\end{array}$ & $\begin{array}{l}\text { Step } \\
\text { sEF }\end{array}$ & $\begin{array}{l}\text { sEF } \\
\text { contribution } \\
\text { to the } \\
\text { process }\end{array}$ & References \\
\hline 1 & IMesPd(acac)Cl & 0.082 & 162.4 & 13.3 & 3.2 & 0.3 & Table S13 \\
\hline 2 & $\mathbf{2 a}$ & 2.186 & 1.5 & 3.3 & 1.3 & 2.8 & Table S20 \\
\hline Total & & 2.186 & & 16.6 & & 3.1 & \\
\hline
\end{tabular}

$$
\begin{gathered}
\mathrm{cEF}=16.6 / 2.186=7.6 \\
\mathrm{sEF}=3.1 / 2.186=1.4
\end{gathered}
$$

According to eq. $\mathrm{S} 38 \mathrm{GWP}\left(\mathrm{Pd}(\mathrm{acac})_{2}\right)=975.8 \mathrm{~g} \mathrm{CO}_{2} / \mathrm{g}(\mathrm{IMes}) \mathrm{Pd}(\mathrm{acac}) \mathrm{Cl}$, thus

$$
\text { GWP IMes Pd acac } \mathrm{Cl}=\frac{975.8 * 0.082}{2.186}=36.6 \frac{\mathrm{gCO}_{2}}{\mathrm{~g} \mathrm{\mathbf {2a }}}
$$




\section{Dehydration of vinyl sulfides to sulfur-functionalized 1,3-butadienes}

\subsection{Choice of dehydrating agent}

Table S22. Screening of the dehydrating agent to produce $\mathbf{3 a}$.<smiles>C=C(Sc1ccccc1)C(C)(C)O</smiles>

$2 a$

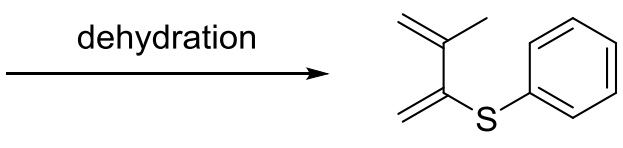

$3 a$

\begin{tabular}{|c|c|c|c|c|c|}
\hline Entry & $\begin{array}{c}\text { Dehydrating } \\
\text { agent }\end{array}$ & Conditions & Solvent & $\begin{array}{c}\text { Conversion of } \\
2 \mathrm{a}, \%\end{array}$ & $\begin{array}{l}\text { Yield of } \\
3 a, \%^{[a]}\end{array}$ \\
\hline \multirow[t]{3}{*}{1} & \multirow[t]{3}{*}{$\mathrm{POCl}_{3}$} & 3 equiv., $0^{\circ} \mathrm{C}, 2 \mathrm{~h}$ & DMF & 92 & 0 \\
\hline & & $\begin{array}{l}1 \text { equiv., } \mathrm{Et}_{3} \mathrm{~N} 1 \text { equiv. } \\
50^{\circ} \mathrm{C}, 1 \mathrm{~h}\end{array}$ & DMF & 90 & 10 \\
\hline & & $\begin{array}{l}3 \text { equiv., } \mathrm{C}_{5} \mathrm{H}_{5} \mathrm{~N} 10 \\
\text { equiv., } 25^{\circ} \mathrm{C}, 4 \mathrm{~h}\end{array}$ & DCM & 55 & 13 \\
\hline \multirow[t]{2}{*}{2} & \multirow[t]{2}{*}{$\mathrm{SOCl}_{2}$} & $\begin{array}{c}1 \text { equiv., } \mathrm{Et}_{3} \mathrm{~N} 1 \text { equiv. } \\
50^{\circ} \mathrm{C}, 1 \mathrm{~h}\end{array}$ & DMF & 87 & 12 \\
\hline & & $\begin{array}{c}3 \text { equiv., } \mathrm{C}_{5} \mathrm{H}_{5} \mathrm{~N} 10 \\
\text { equiv., } 0^{\circ} \mathrm{C}, 2 \mathrm{~h}\end{array}$ & DCM & 84 & 0 \\
\hline 3 & $\mathrm{HCOOH}$ & 10 equiv., $25^{\circ} \mathrm{C}, 3 \mathrm{~h}$ & DCM & 100 & 0 \\
\hline 4 & $\mathrm{CF}_{3} \mathrm{COOH}$ & 10 equiv., $0^{\circ} \mathrm{C}, 1,5 \mathrm{~h}$ & DCM & 100 & 0 \\
\hline 5 & $\mathrm{HCl}$ & $10 \mathrm{~mol} . \%, 25^{\circ} \mathrm{C}, 2 \mathrm{~h}$ & $\mathrm{CHCl}_{3}$ & 100 & 0 \\
\hline \multirow[t]{2}{*}{6} & \multirow[t]{2}{*}{$\mathrm{H}_{2} \mathrm{SO}_{4}$} & \multirow[t]{2}{*}{1 equiv., $25^{\circ} \mathrm{C}, 1 \mathrm{~h}$} & $\mathrm{MeCN}$ & 100 & 0 \\
\hline & & & $\mathrm{EtOH}$ & 100 & 0 \\
\hline 7 & $\mathrm{MsCl}$ & $\begin{array}{c}3 \text { equiv., } \mathrm{Et}_{3} \mathrm{~N} 7 \text { equiv., } \\
0^{\circ} \mathrm{C}, 0.5 \mathrm{~h}\end{array}$ & - & 95 & 27 \\
\hline \multirow[t]{3}{*}{8} & \multirow[t]{3}{*}{ Amberlyst } & $10 \%, 80^{\circ} \mathrm{C}, 4 \mathrm{~h}$ & - & 100 & 0 \\
\hline & & $5 \%, 60^{\circ} \mathrm{C}, 18 \mathrm{~h}$ & - & 37 & 0 \\
\hline & & $5 \%, 60{ }^{\circ} \mathrm{C}, 18 \mathrm{~h}$ & EtOAc & 100 & 0 \\
\hline \multirow[t]{2}{*}{9} & \multirow[t]{2}{*}{$\mathrm{KOH}$} & 3 equiv., $60^{\circ} \mathrm{C}, 18 \mathrm{~h}$ & $\begin{array}{l}\mathrm{EtOH} \\
96 \%\end{array}$ & 23 & 0 \\
\hline & & 1 equiv., $60^{\circ} \mathrm{C}, 18 \mathrm{~h}$ & iPrOH & 10 & 0 \\
\hline
\end{tabular}


Table S23. Screening of ionic liquids for dehydration of $\mathbf{2 a}$ into $\mathbf{3 a}$.

\begin{tabular}{|c|c|c|c|c|c|}
\hline \multicolumn{4}{|r|}{$\frac{\mathrm{P}_{2} \mathrm{O}_{5}}{\text { Solvent }}$} & $3 a$ & \multirow[b]{2}{*}{ Yield of $3 a, \%^{[a]}$} \\
\hline Entry & Additives & Solvent & Temperature & Conversion of $2 \mathrm{a}, \%$ & \\
\hline \multicolumn{6}{|c|}{ Conditions: $2 \mathrm{a} 0.5 \mathrm{mmol}, \mathrm{P}_{2} \mathrm{O}_{5} 0.5 \mathrm{mmol}$, ionic liquid $5 \mathrm{mmol}, 10 \mathrm{~min}$} \\
\hline 1 & \multirow[t]{9}{*}{-} & [EMIM]Cl & $90^{\circ} \mathrm{C}$ & 0 & 0 \\
\hline 2 & & [BMIM]Cl & $50^{\circ} \mathrm{C}$ & 0 & 0 \\
\hline 3 & & {$\left[\mathrm{BMIM}_{\mathrm{PF}} 6\right.$} & $50^{\circ} \mathrm{C}$ & 100 & 0 \\
\hline 4 & & {$\left[\mathrm{BMIM}_{\mathrm{B}} \mathrm{BF}_{4}\right.$} & $50^{\circ} \mathrm{C}$ & 100 & 0 \\
\hline 5 & & {$[\mathrm{EMIM}] \mathrm{SO}_{4} \mathrm{H}$} & $70^{\circ} \mathrm{C}$ & 100 & 23 \\
\hline 6 & & {$[\mathrm{BMIM}] \mathrm{SO}_{4} \mathrm{H}$} & $90^{\circ} \mathrm{C}$ & 100 & 30 \\
\hline 7 & & {$[\mathrm{EMIM}] \mathrm{SO}_{4} \mathrm{Me}$} & $50^{\circ} \mathrm{C}$ & 100 & 44 \\
\hline 8 & & {$[\mathrm{EMIM}] \mathrm{SO}_{4} \mathrm{Et}$} & $50^{\circ} \mathrm{C}$ & 100 & 40 \\
\hline 9 & & {$[\mathrm{EMIM}] \mathrm{SO}_{4} \mathrm{Me}$} & r.t. & 90 & 27 \\
\hline
\end{tabular}




\subsection{Evaluation of $\mathrm{CEF}$ for vinyl sulfides dehydration}

Step 1

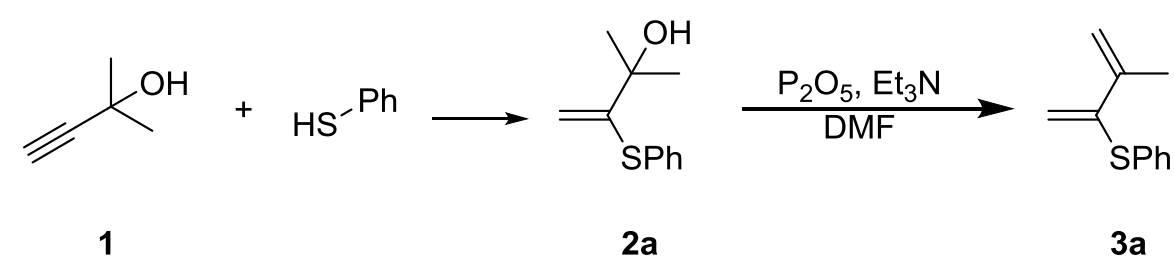

Scheme S4. Synthesis of diene $3 a$.

Table S24. Material Input-Output for the Dehydration of Vinyl Sulfides with Tertiary Hydroxyl Group with product purification by extraction.

\begin{tabular}{|c|c|c|c|c|c|c|c|c|}
\hline Step & $\begin{array}{l}\text { Input } \\
\text { material }\end{array}$ & $\begin{array}{l}\text { Input } \\
\text { type }\end{array}$ & $\begin{array}{l}\text { Output } \\
\text { Material }\end{array}$ & $\begin{array}{c}\mathrm{Mw} \\
(\mathrm{g} / \mathrm{mol})\end{array}$ & Equiv. & $\begin{array}{l}\text { Input } \\
\text { (mol) }\end{array}$ & $\begin{array}{c}\text { Input } \\
\text { Weight }\end{array}$ & $\begin{array}{l}\text { Output } \\
\text { Weight }\end{array}$ \\
\hline \multirow{5}{*}{ 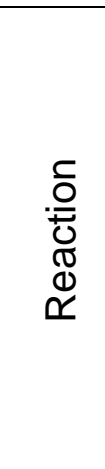 } & $2 a$ & $\begin{array}{c}\text { Raw } \\
\text { material }\end{array}$ & & 194.29 & 1 & 0.001 & 0.194 & \\
\hline & $\mathrm{P}_{2} \mathrm{O}_{5}$ & $\begin{array}{c}\text { Raw } \\
\text { material }\end{array}$ & & 141.94 & 2 & 0.002 & 0.284 & \\
\hline & $\mathrm{Et}_{3} \mathrm{~N}$ & $\begin{array}{c}\text { Raw } \\
\text { material }\end{array}$ & & 101.19 & 1 & 0.001 & 0.101 & \\
\hline & DMF & Solvent & & & & & 1.416 & \\
\hline & & $\begin{array}{l}\text { Crude } \\
\text { product }\end{array}$ & $3 a$ & 176.28 & & & & $0.173^{[a}$ \\
\hline \multirow{8}{*}{ 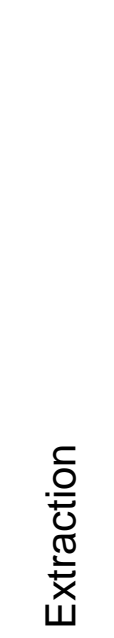 } & $3 a$ & $\begin{array}{l}\text { Crude } \\
\text { product }\end{array}$ & & 176.28 & & & 0.173 & \\
\hline & $\mathrm{K}_{2} \mathrm{CO}_{3}^{[\mathrm{b}]}$ & Workup & & 138.21 & & 0.0557 & 7.70 & \\
\hline & $\mathrm{H}_{2} \mathrm{O}^{[\mathrm{b}]}$ & Solvent & & & & & 7.70 & \\
\hline & $\begin{array}{l}\text { Petroleum } \\
\text { ether }^{[c]}\end{array}$ & Solvent & & & & & 23.45 & \\
\hline & $\mathrm{NaCl}^{[\mathrm{d}]}$ & Workup & & 58.4 & & 0.0393 & 2.295 & \\
\hline & $\mathrm{H}_{2} \mathrm{O}^{[\mathrm{d}]}$ & Solvent & & & & & 9.183 & \\
\hline & $\mathrm{Na}_{2} \mathrm{SO}_{4}{ }^{[\mathrm{e}]}$ & Workup & & 142.04 & & & 0.018 & \\
\hline & & Product & $3 a$ & 176.28 & & & & 0.166 \\
\hline
\end{tabular}

[a] Yield of the product was determined by ${ }^{1} \mathrm{H}$ NMR $(98 \%)$. ${ }^{[b]}$ Parameters of saturated solution of $\mathrm{K}_{2} \mathrm{CO}_{3}(10 \mathrm{~mL})$ are $\mathrm{d}=1.5404 \mathrm{~g} / \mathrm{mL}, \mathrm{C}=5.573 \mathrm{M}$. ${ }^{[\mathrm{c}]} \mathrm{d}($ petroleum ether) $=0.67 \mathrm{~g} / \mathrm{mL}$. [d] Parameters of saturated solution of $\mathrm{NaCl}(10 \mathrm{~mL})$ are $\mathrm{d}=1.1478 \mathrm{~g} / \mathrm{mL}, \mathrm{c}=3.928 \mathrm{M}$. ${ }^{[\mathrm{e}]}$ Based on water solubility in hexane $0.099 \mathrm{~g}$ in $100 \mathrm{~g}$.

$$
\begin{aligned}
& \text { EF step } 2=\frac{0.194+0.284+0.101+1.416 * 0.1}{0.166}+ \\
& +\frac{7.70+23.45 * 0.1+2.295+0.018-0.166}{0.166}=77.7
\end{aligned}
$$




$$
\begin{gathered}
\text { cEF step } 2=\frac{0.194+0.284+0.101+1.416}{0.166}+ \\
+\frac{7.70+7.70+23.45+2.295+9.183+0.018-0.166}{0.166}=314.3 \\
\text { SEF reaction }=\frac{0.194+0.284+0.101-0.173}{0.173}=2.3 \\
\text { EF extraction }=\frac{0.173+7.70+23.45 * 0.1+2.295+0.018-0.166}{0.166}=74.5 \\
c E F \text { extraction }=\frac{0.173+7.74+7.66+23.45+2.295+9.183+0.018-0.166}{0.166} \\
=303.3
\end{gathered}
$$

Table S25. cEF contribution to the synthesis of $\mathbf{2 a}$ with purification by extraction (Scheme S4).

\begin{tabular}{|c|c|c|c|c|c|c|c|c|}
\hline Step & $\begin{array}{c}\text { Input } \\
\text { material }\end{array}$ & Input type & $\begin{array}{l}\text { Output } \\
\text { Material }\end{array}$ & $\begin{array}{c}\text { Mw } \\
(\mathrm{g} / \mathrm{mol})\end{array}$ & Equiv. & $\begin{array}{l}\text { Input } \\
\text { (mol) }\end{array}$ & $\begin{array}{l}\text { Input } \\
\text { Weight }\end{array}$ & $\begin{array}{l}\text { Output } \\
\text { Weight }\end{array}$ \\
\hline \multirow{4}{*}{$\begin{array}{l}\frac{}{0} \\
\stackrel{0}{0} \\
\mathbb{0} \\
\stackrel{\Phi}{\simeq}\end{array}$} & $2 a$ & $\begin{array}{c}\text { Raw } \\
\text { material }\end{array}$ & & 194.29 & 1 & 0.010 & 1.943 & \\
\hline & $\mathrm{P}_{2} \mathrm{O}_{5}$ & $\begin{array}{c}\text { Raw } \\
\text { material }\end{array}$ & & 141.94 & 2 & 0.020 & 2.839 & \\
\hline & $\mathrm{Et}_{3} \mathrm{~N}$ & $\begin{array}{c}\text { Raw } \\
\text { material }\end{array}$ & & 101.19 & 1 & 0.010 & 1.012 & \\
\hline & DMF & Solvent & & & & & 7.556 & \\
\hline \multirow{2}{*}{ 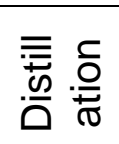 } & Acetone $^{[\mathrm{a}]}$ & Solvent & & & & & 0.458 & \\
\hline & & Product & $3 a$ & 176.28 & & & & $0.9348^{[b]}$ \\
\hline
\end{tabular}

\begin{tabular}{|c|l|l|l|l|l|}
\hline Step \# & Product & $\begin{array}{l}\text { Output } \\
\text { weight }\end{array}$ & Step cEF & $\begin{array}{l}\text { cEF } \\
\text { contribution to } \\
\text { the process }\end{array}$ & References \\
\hline 1 & $\mathbf{2 a}$ & 0.194 & 3.1 & 0.6 & Table S16 \\
\hline 2 & $\mathbf{3 a}$ & 0.166 & 314 & 52.2 & Table S24 \\
\hline Total & & 0.166 & & 52.8 & \\
\hline
\end{tabular}

$$
\mathrm{cEF}=52.8 / 0.166=318.1
$$

Table S26. Material Input-Output for the Dehydration of Vinyl Sulfides with Tertiary Hydroxyl Group with product purification by distillation.

[a] To avoid additional product loss acetone was used to wash the product from the distillation 
unit. ${ }^{[b]}$ Yield of the product after product purification was $56 \%$.

$$
\begin{array}{cc}
\text { EF } \text { step } 2=\frac{1.943+2.839+1.012+7.556 * 0.1+0.458 * 0.1-0.9348}{0.9348}=6.1 & \text { (Eq. S79) } \\
\text { cEF step } 2=\frac{1.943+2.839+1.012+7.556+0.458-0.9348}{0.9348}=13.8 & \text { (Eq. S80) }
\end{array}
$$

Table S27. cEF contribution to the synthesis of 3a with purification by distillation (Scheme S4).

\begin{tabular}{|c|l|l|l|l|l|}
\hline Step \# & Product & $\begin{array}{l}\text { Output } \\
\text { weight }\end{array}$ & Step cEF & $\begin{array}{l}\text { cEF } \\
\text { contribution to } \\
\text { the process }\end{array}$ & References \\
\hline 1 & $\mathbf{2 a}$ & 1.943 & 3.1 & 6.0 & Table S16 \\
\hline 2 & $\mathbf{3 a}$ & 0.9348 & 14 & 12.9 & Table S26 \\
\hline Total & & & & 18.9 & \\
\hline
\end{tabular}

$$
c E F=18.90 .9348=20.2
$$




\section{Assessment of green metrics of synthesis of 4 .}

\subsection{One-Pot synthesis of 4 .}

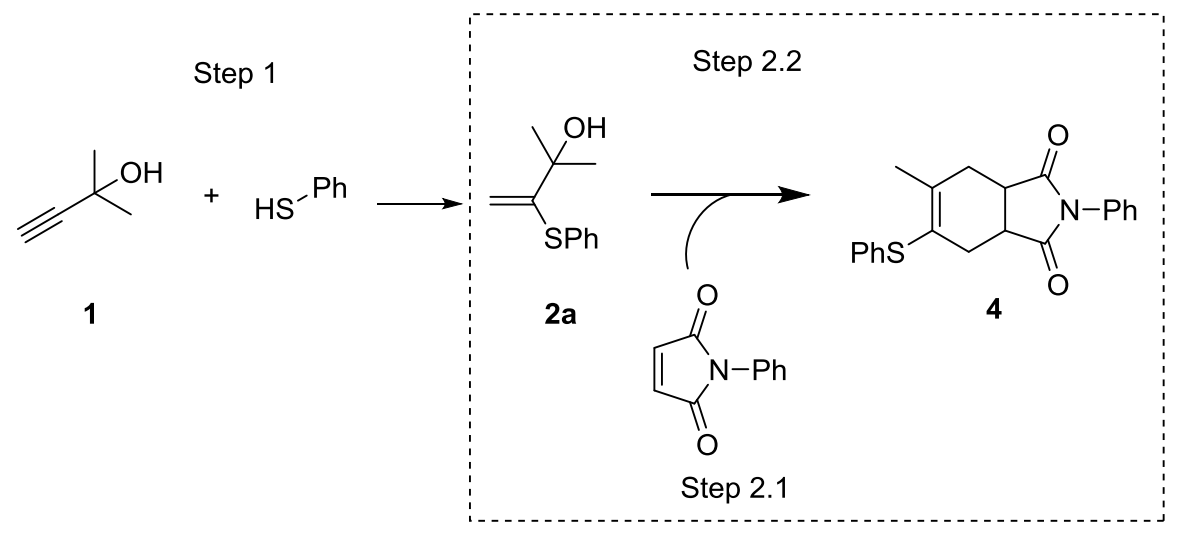

Scheme S5. Synthesis of 4.

\section{E-factor of Step 2.1}

Due to the commercial price of $\mathrm{N}$-phenylmaleimide higher over $100 \$ / \mathrm{mol}$, it cannot be considered as a starting material. cEF of synthesis of the N-phenylmaleimide was calculated on the basis of the literature procedure: ${ }^{[14]}$

"A. Maleanilic acid. In a 5 I three-necked flask provided with a paddle-type stirrer, a reflux condenser, and a dropping funnel are placed $196 \mathrm{~g}$. (2 moles) of maleic anhydride and $2.5 \mathrm{I}$. of ethyl ether. The stirrer is started and, when all the maleic anhydride has dissolved, a solution of $182 \mathrm{ml}$. (186 g., 2 moles) of aniline in $200 \mathrm{ml}$. of ether is run in through the dropping funnel. The resulting thick suspension is stirred at room temperature for 1 hour and is then cooled to $15-20^{\circ}$ in an ice bath. The product is obtained by suction filtration. It is a fine, cream-colored powder, m.p. $201-202^{\circ}$, suitable for use in the next step without purification. The yield is $371-374 \mathrm{~g}$. (97-98\%).

B. N-Phenylmaleimide. In a 2-I. Erlenmeyer flask are placed $670 \mathrm{ml}$. of acetic anhydride and $65 \mathrm{~g}$. of anhydrous sodium acetate. The maleanilic acid (316 g.), obtained as described above, is added, and the resulting suspension is dissolved by swirling and heating on a steam bath for 30 minutes. The reaction mixture is cooled almost to room temperature in a cold water bath and is then poured into $1.3 \mathrm{I}$. of ice water. The precipitated product is removed by suction filtration, washed three times with $500-\mathrm{ml}$. portions of ice-cold water and once with $500 \mathrm{ml}$. of petroleum ether (b.p. $30-60^{\circ}$ ), and dried. The yield of crude Nphenylmaleimide is $214-238$ g. $(75-80 \%)$, m.p. $88-89^{\circ}$. Recrystallization from cyclohexane gives canary-yellow needles, m.p. 89-89.8 ${ }^{\circ}$.

About $500 \mathrm{ml}$. of the refluxing solvent will dissolve some $58 \mathrm{~g}$. of $\mathrm{N}$-phenylmaleimide. The recovery of recrystallized material is approximately $93 \%$." 
Table S28. Material Input-Output of Step 2.1A (Scheme S5).

\begin{tabular}{|c|c|c|c|c|c|c|}
\hline & Input material & $\begin{array}{l}\text { Input } \\
\text { type }\end{array}$ & $\begin{array}{l}\text { Output } \\
\text { Material }\end{array}$ & $\begin{array}{l}\text { Input } \\
\text { (mol) }\end{array}$ & $\begin{array}{l}\text { Input } \\
\text { Weight }\end{array}$ & $\begin{array}{l}\text { Output } \\
\text { Weight }\end{array}$ \\
\hline \multirow{3}{*}{ 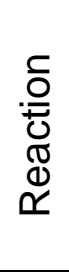 } & $\begin{array}{c}\text { Maleic } \\
\text { anhydride }\end{array}$ & $\begin{array}{c}\text { Raw } \\
\text { material }\end{array}$ & & 2 & 196 & \\
\hline & $\mathrm{Et}_{2} \mathrm{O}$ & Solvent & & & 1925.1 & \\
\hline & Aniline & $\begin{array}{c}\text { Raw } \\
\text { material }\end{array}$ & & 2 & 186 & \\
\hline & & Product & $\begin{array}{l}\text { Maleanilic } \\
\text { acid }\end{array}$ & & & 374 \\
\hline
\end{tabular}

$$
\mathrm{cEF} \text { step 2.1A }=5.2
$$

Table S29. Material Input-Output of Step 2.1B (Scheme S5).

\begin{tabular}{|c|c|c|c|c|c|}
\hline & $\begin{array}{l}\text { Input } \\
\text { material }\end{array}$ & Input type & Output Material & $\begin{array}{c}\text { Input } \\
\text { Weight }\end{array}$ & $\begin{array}{l}\text { Output } \\
\text { Weight }\end{array}$ \\
\hline \multirow{3}{*}{ 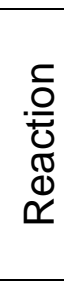 } & $\begin{array}{c}\text { Acetic } \\
\text { anhydride }\end{array}$ & Raw material & & 620.4 & \\
\hline & $\mathrm{NaOAc}$ & Raw material & & 65 & \\
\hline & $\begin{array}{l}\text { Maleanic } \\
\text { acid }\end{array}$ & Raw material & & 316 & \\
\hline \multirow{5}{*}{ 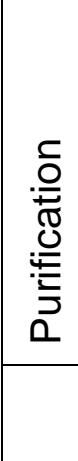 } & Water & Solvent & & 2777.6 & \\
\hline & $\begin{array}{l}\text { Petroleum } \\
\text { ether }\end{array}$ & Solvent & & 325 & \\
\hline & & Product & $\begin{array}{c}\mathrm{N}- \\
\text { Phenylmaleimide } \\
\end{array}$ & & 238 \\
\hline & Cyclohexane & $\begin{array}{c}\text { Solvent } \\
\text { (recrystallization) }\end{array}$ & & 1598.5 & \\
\hline & & Product & $\begin{array}{c}\mathrm{N}- \\
\text { Phenylmaleimide }\end{array}$ & & 221 \\
\hline
\end{tabular}

$$
\mathrm{CEF}_{\text {step } 2.1 \mathrm{~B}}=24.8
$$


Table S30. cEF and SEF Contribution to the N-Phenylmaleimide synthesis (Step 2.1 on Scheme S5).

\begin{tabular}{|l|l|l|l|l|l|}
\hline $\begin{array}{l}\text { Step } \\
\#\end{array}$ & Product & $\begin{array}{l}\text { Output } \\
\text { weight }\end{array}$ & $\begin{array}{l}\text { Step } \\
\text { cEF }\end{array}$ & $\begin{array}{l}\text { cEF } \\
\text { contribution } \\
\text { to the } \\
\text { process }\end{array}$ & Reference \\
\hline 2.1A & Maleanilic acid & 316 & 5.2 & 1643.2 & Table S28 \\
\hline $2.1 \mathrm{~B}$ & $\begin{array}{l}\text { N- } \\
\text { Phenylmaleimide }\end{array}$ & 221 & 24.8 & 5480.8 & Table S29 \\
\hline Total & & 221 & & 7124 & \\
\hline
\end{tabular}

$$
\text { cEF step } 2.1=7124221=32.2^{g} g
$$

\section{E-factor of Step 2.2}

Table S31. Material Input-Output for one-pot synthesis of 4.

\begin{tabular}{|c|c|c|c|c|c|c|c|c|}
\hline Step & Input material & Input type & $\begin{array}{c}\text { Output } \\
\text { Material }\end{array}$ & $\begin{array}{c}\mathrm{Mw} \\
(\mathrm{g} / \mathrm{mol})\end{array}$ & Equiv. & $\begin{array}{c}\text { Input } \\
\text { (mmol) }\end{array}$ & $\begin{array}{c}\text { Input } \\
\text { Weight }\end{array}$ & $\begin{array}{l}\text { Output } \\
\text { Weight }\end{array}$ \\
\hline \multirow{6}{*}{ 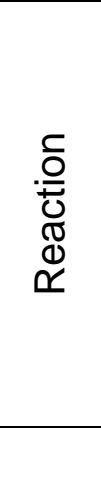 } & $2 a$ & $\begin{array}{c}\text { Raw } \\
\text { material }\end{array}$ & & 194.29 & 1 & 1 & 0.194 & \\
\hline & $\mathrm{P}_{2} \mathrm{O}_{5}$ & Reagent & & 141.94 & 2 & 2 & 0.284 & \\
\hline & $\mathrm{Et}_{3} \mathrm{~N}$ & Reagent & & 101.19 & 1 & 1 & 0.101 & \\
\hline & DMF & Solvent & & & & & 1.416 & \\
\hline & $\begin{array}{c}\mathrm{N}- \\
\text { phenylmaleimide }\end{array}$ & $\begin{array}{c}\text { Raw } \\
\text { material }\end{array}$ & & & 3 & 3 & 0.520 & \\
\hline & & Product & 4 & 349.45 & & & & 0.332 \\
\hline \multirow{6}{*}{ 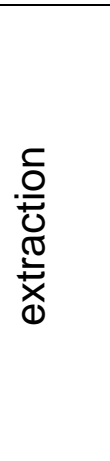 } & $\mathrm{K}_{2} \mathrm{CO}_{3}{ }^{[\mathrm{a}]}$ & Workup & & 138.21 & & 0.057 & 7.87 & \\
\hline & $\mathrm{H}_{2} \mathrm{O}^{[\mathrm{a}]}$ & Solvent & & & & & 7.53 & \\
\hline & Ethyl acetate & solvent & & & & & 27.06 & \\
\hline & $\mathrm{NaCl}^{[\mathrm{a}]}$ & Workup & & 58.4 & & 0.039 & 2.294 & \\
\hline & $\mathrm{H}_{2} \mathrm{O}^{[\mathrm{a}]}$ & Solvent & & & & & 9.184 & \\
\hline & $\mathrm{Na}_{2} \mathrm{SO}_{4}{ }^{[\mathrm{b}]}$ & Workup & & 142.04 & & & 0.018 & \\
\hline \multirow{3}{*}{$\geq \frac{\xi}{\bar{D}}$} & Silica gel ${ }^{[c]}$ & Workup & & & & & $27.45^{[c]}$ & \\
\hline & Eluent ${ }^{[c]}$ & Solvent & & & & & 224.01 & \\
\hline & & Product & 4 & 349.45 & & & & 0.259 \\
\hline
\end{tabular}


Parameters of saturated solution of $\mathrm{NaCl}(10 \mathrm{~mL})$ are $\mathrm{d}=1.1478 \mathrm{~g} / \mathrm{mL}, \mathrm{C}=3.928 \mathrm{M}$. ${ }^{[\mathrm{c}]}$ Amount of silica gel and solvent was considered according to the Table 2. Average weight of the eluent $(\mathrm{V}$ (petroleum ether) $/ \mathrm{V}$ (ethyl acetate) $=1: 1)$ was used for $285 \mathrm{ml}$.

$$
\mathrm{cEF}_{\text {step } 2}=1187.9
$$

Table S32. cEF Contribution to the One-Pot synthesis of 4 (Scheme S5).

\begin{tabular}{|c|c|c|c|c|c|}
\hline Step \# & Product & $\begin{array}{l}\text { Output } \\
\text { weight }\end{array}$ & Step cEF & $\begin{array}{l}\text { CEF } \\
\text { contribution to } \\
\text { the process }\end{array}$ & Reference \\
\hline 1 & $2 a$ & 0.194 & 3.1 & 0.6 & Table S16 \\
\hline 2.1 & N-Phenylmaleimide & 0.520 & 32.2 & 16.7 & Table S30 \\
\hline 2.2 & 4 & 0.259 & 1188 & 307.7 & Table S31 \\
\hline Total & & 0.259 & & 325.0 & \\
\hline
\end{tabular}

$$
c E F=325.0 \quad 0.259^{2}=1254.8^{g} g
$$

\subsection{Sequential synthesis of 4.}

Step 1

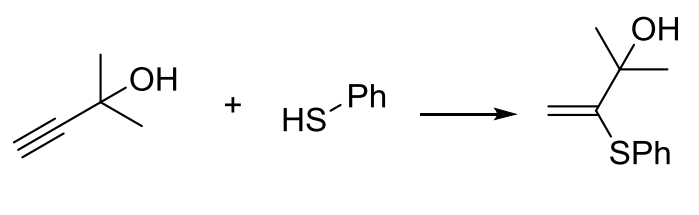

1

$2 a$
Step 2

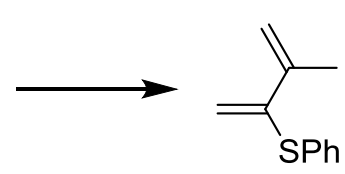

$3 \mathbf{a}$
Step 3.2
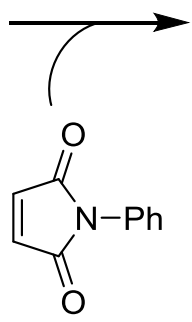

Step 3.1

Scheme S6. Sequential synthesis of 4. 
Table S33. Material Input-Output for Step 3.2 (Scheme S6).

\begin{tabular}{|c|c|c|c|c|c|c|c|c|}
\hline Step & Input material & Input type & $\begin{array}{l}\text { Output } \\
\text { Material }\end{array}$ & $\begin{array}{c}\mathrm{Mw} \\
(\mathrm{g} / \mathrm{mol})\end{array}$ & Equiv. & $\begin{array}{l}\text { Input } \\
\text { (mmol) }\end{array}$ & $\begin{array}{l}\text { Input } \\
\text { Weight }\end{array}$ & $\begin{array}{l}\text { Output } \\
\text { Weight }\end{array}$ \\
\hline \multirow{3}{*}{ 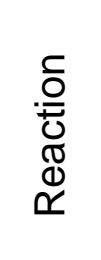 } & $3 a$ & $\begin{array}{c}\text { Raw } \\
\text { material }\end{array}$ & & 176.28 & 1 & 1 & 0.176 & \\
\hline & $\begin{array}{c}\mathrm{N}- \\
\text { phenylmaleimide }\end{array}$ & $\begin{array}{c}\text { Raw } \\
\text { material }\end{array}$ & & 173.17 & 3 & 3 & 0.519 & \\
\hline & DMF & Solvent & & & & & 0.944 & \\
\hline \multirow{3}{*}{ ㅎํ응 } & Silica ge $\mathrm{l}^{[\mathrm{a}]}$ & Workup & & & & & 23.85 & \\
\hline & Eluent ${ }^{[a]}$ & Solvent & & & & & 212.2 & \\
\hline & & Product & 4 & 349.45 & & & & 0.332 \\
\hline
\end{tabular}

${ }^{[a]}$ Amount of silica gel and solvent was considered according to the Table 2. Average weight of the eluent $(\mathrm{V}$ (petroleum ether) $/ \mathrm{V}$ (ethyl acetate $)=1: 1$ ) was used for $270 \mathrm{ml}$.

$$
\begin{aligned}
\mathrm{EF}_{\text {step 3 }} & =137.1 \\
\mathrm{CEF} & =714.9
\end{aligned}
$$

Table S34. cEF Contribution to the Sequential Synthesis of 4 (Scheme S6).

\begin{tabular}{|c|l|l|l|l|l|}
\hline Step \# & Product & $\begin{array}{l}\text { Output } \\
\text { weight }\end{array}$ & Step cEF & $\begin{array}{l}\text { cEF } \\
\text { contribution to } \\
\text { the process }\end{array}$ & Reference \\
\hline $1-2$ & $\mathbf{3 a}$ & 0.176 & 20.4 & 3.6 & Table S27 \\
\hline 3.1 & $\begin{array}{l}\mathrm{N}- \\
\text { Phenylmaleimide }\end{array}$ & 0.519 & 32.2 & 16.7 & Table S30 \\
\hline 3.2 & $\mathbf{4}$ & 0.332 & 714.9 & 237.3 & Table S33 \\
\hline Total & & 0.332 & & 257.6 & \\
\hline
\end{tabular}

$$
c E F=257.60 .332=775.9^{g} g
$$


NMR spectra of dehydration reaction and Diels-Alder reaction products

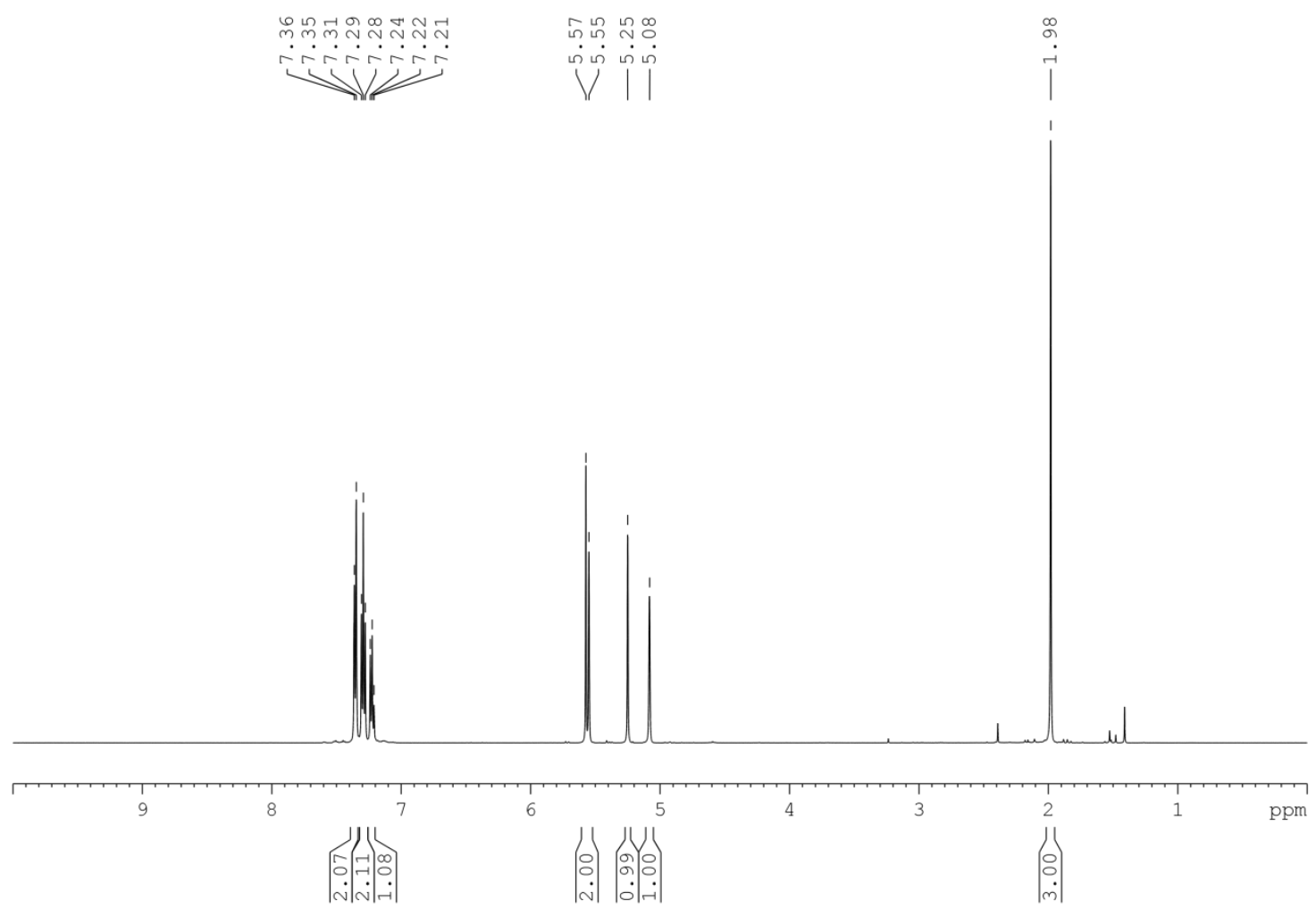

Figure S1. ${ }^{1} \mathrm{H}$ NMR spectrum of 3a (3-methylbuta-1,3-dien-2-yl)(phenyl)sulfane in $\mathrm{CDCl}_{3}$.

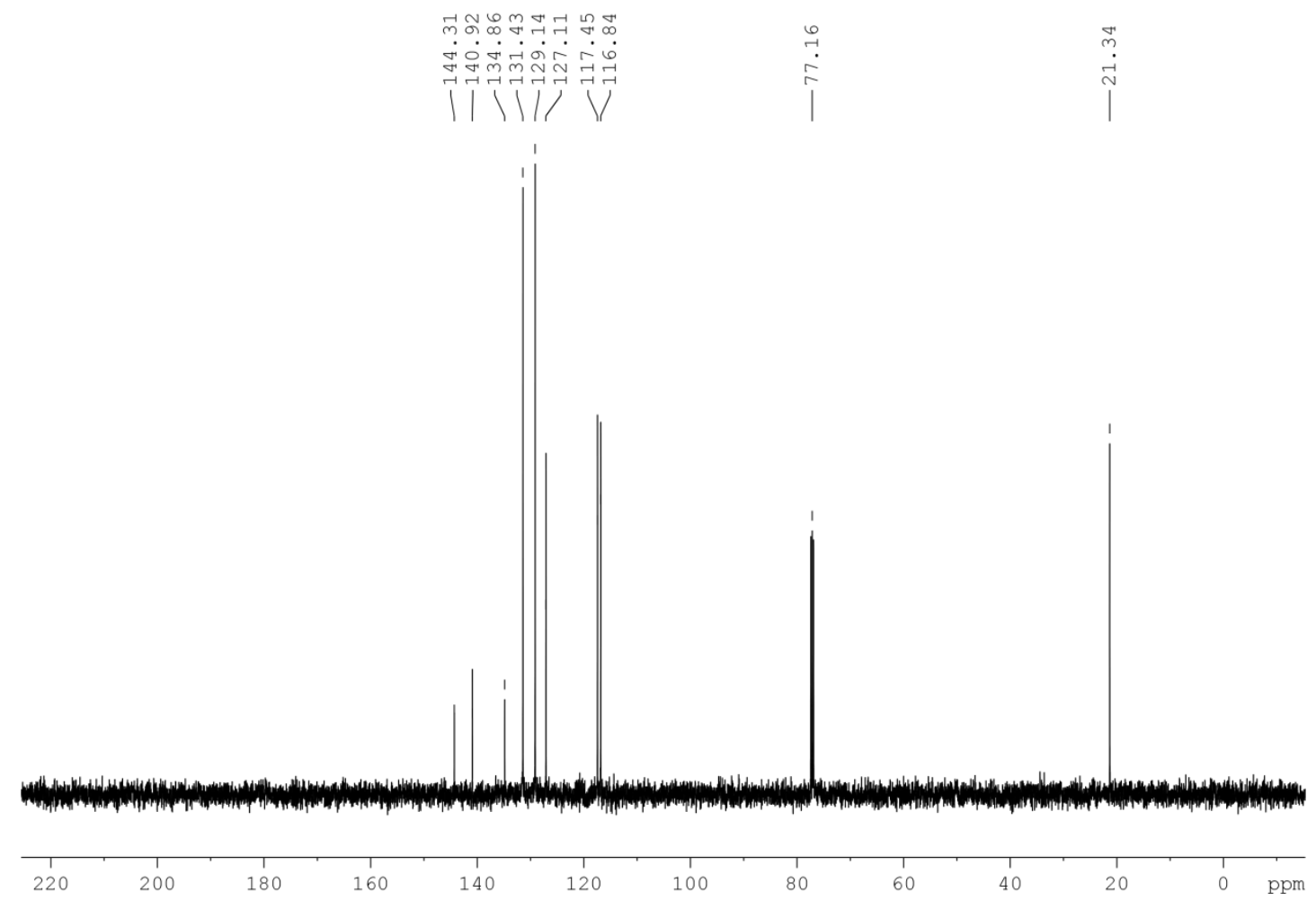

Figure S2. ${ }^{13} \mathrm{C}\left\{{ }^{1} \mathrm{H}\right\}$ NMR spectrum of 3a (3-methylbuta-1,3-dien-2-yl)(phenyl)sulfane in $\mathrm{CDCl}_{3}$. 


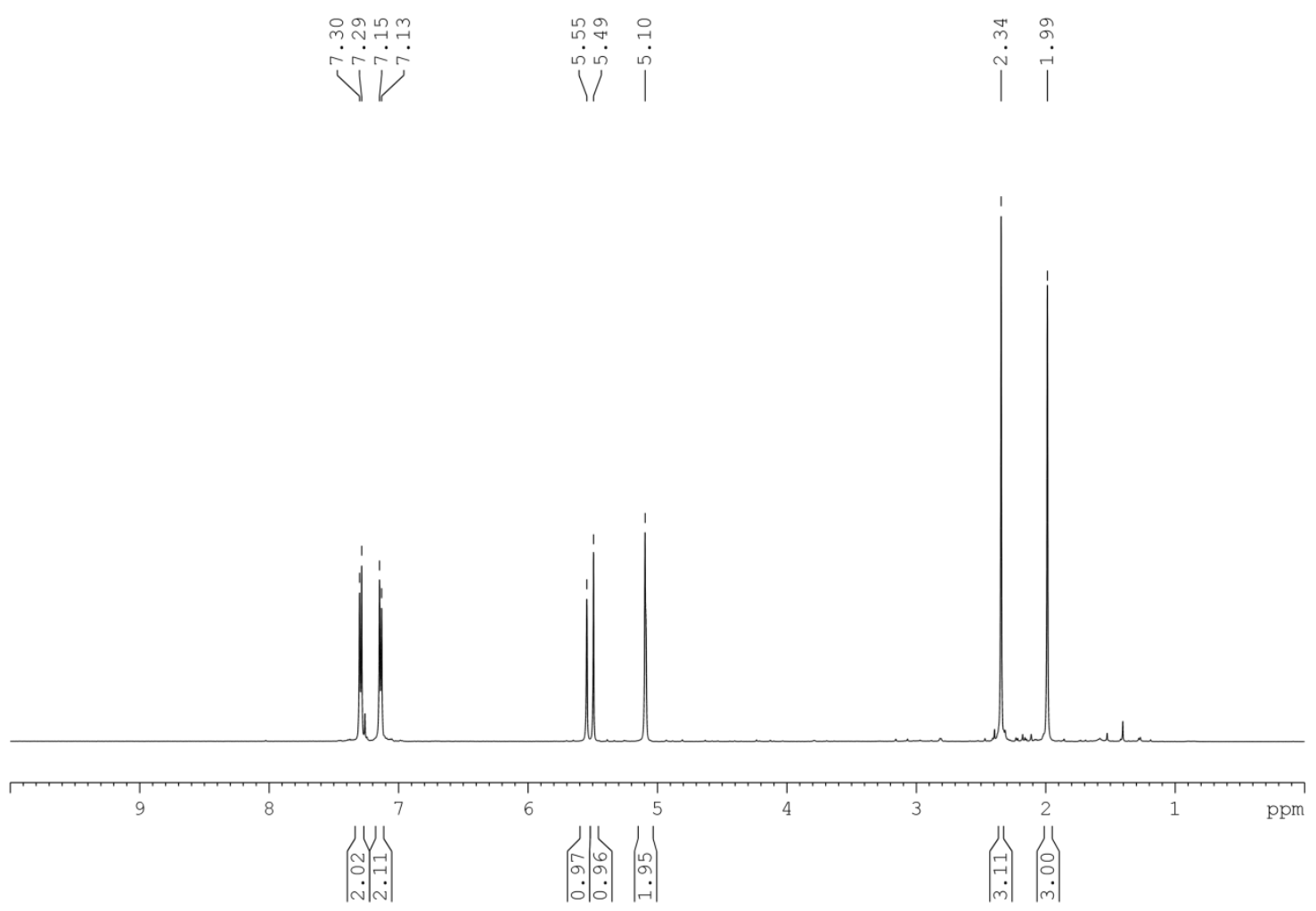

Figure S3. ${ }^{1} \mathrm{H}$ NMR spectrum of $\mathbf{3 b}$ (4-methylbuta-1,3-dien-2-yl)(p-tolyl)sulfane in $\mathrm{CDCl}_{3}$.
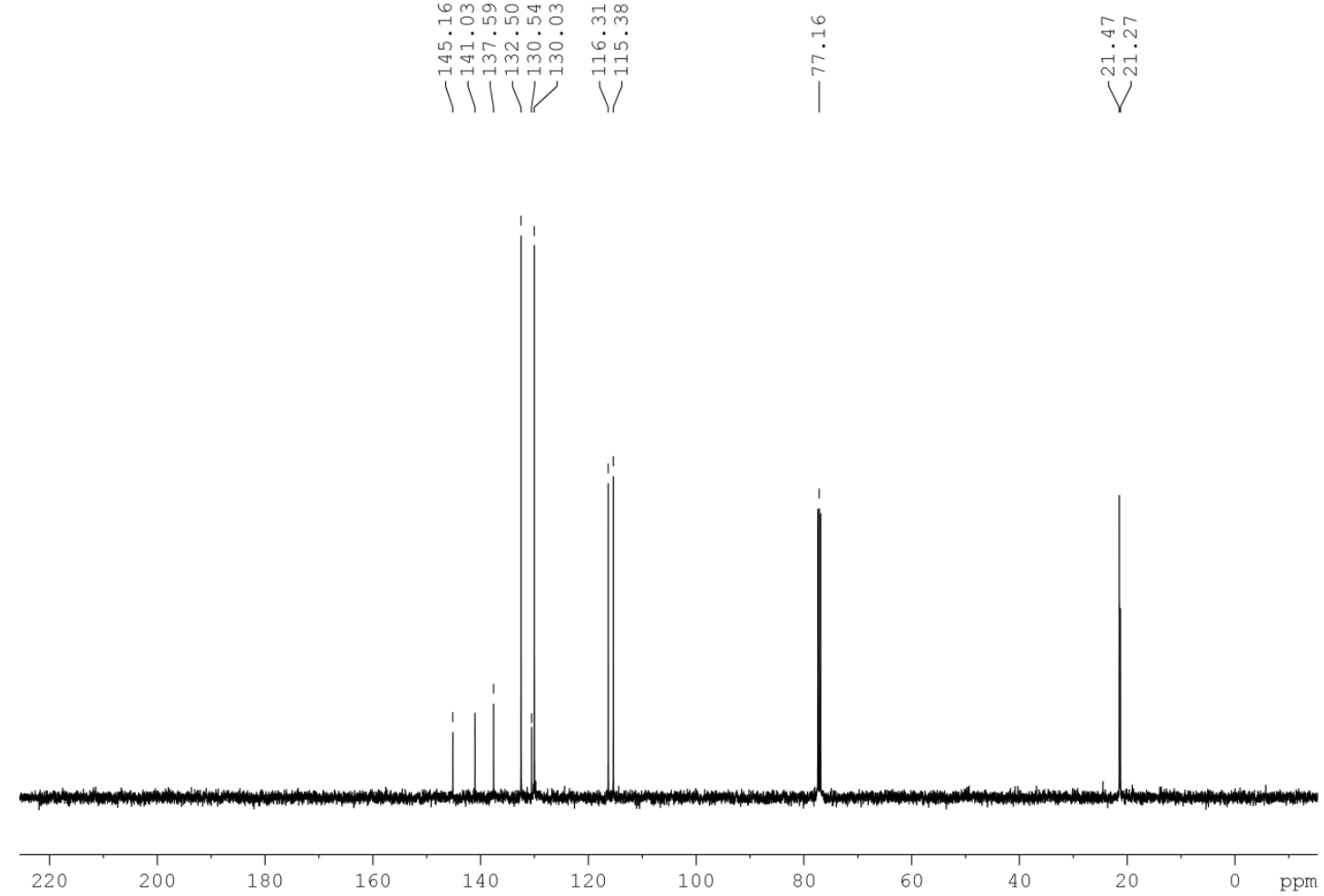

Figure S4. ${ }^{13} \mathrm{C}\left\{{ }^{1} \mathrm{H}\right\}$ NMR spectrum of $\mathbf{3 b}$ (4-methylbuta-1,3-dien-2-yl)(p-tolyl)sulfane in $\mathrm{CDCl}_{3}$. 


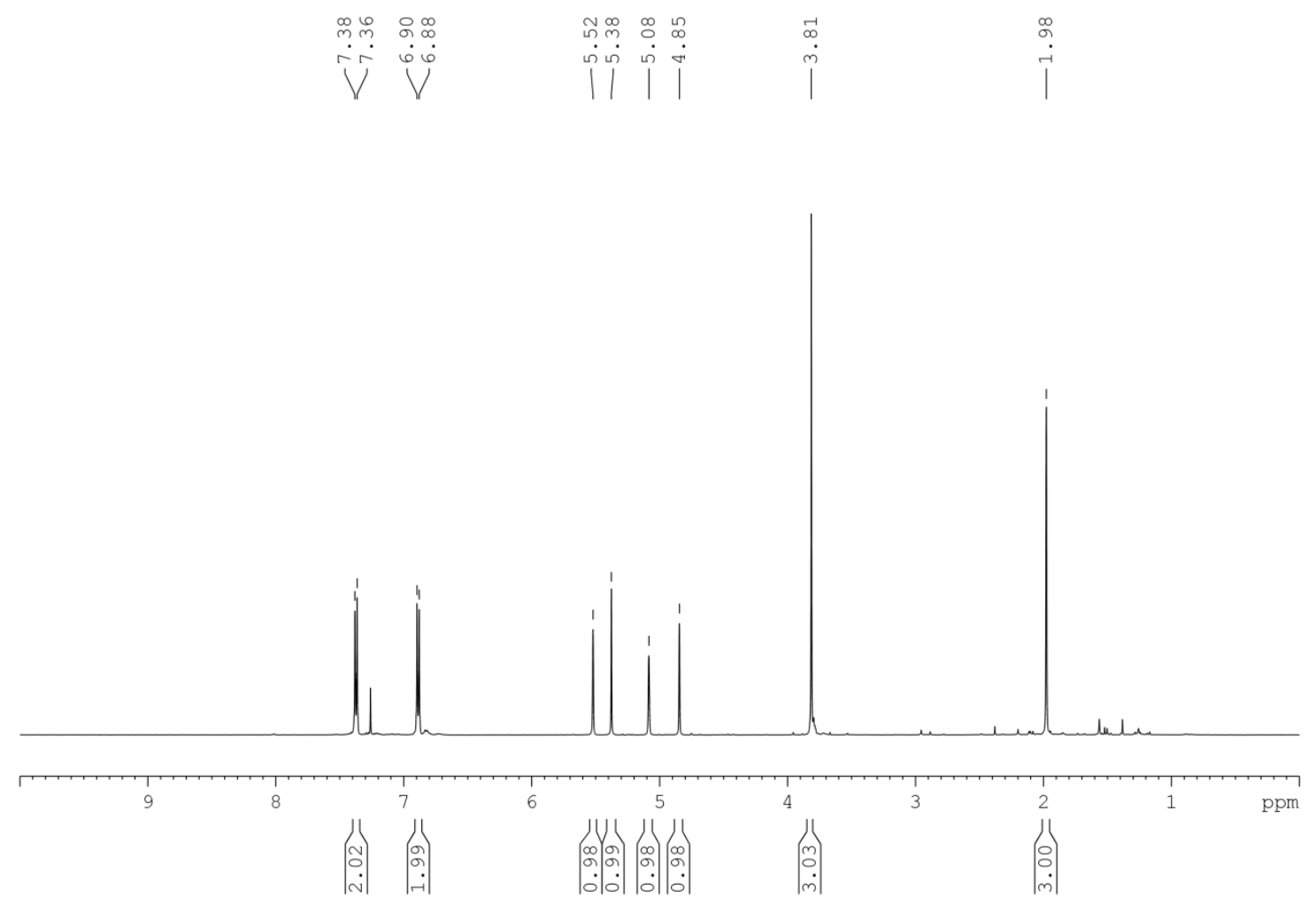

Figure S5. ${ }^{1} \mathrm{H}$ NMR spectrum of 3c (4-methoxyphenyl)(3-methylbuta-1,3-dien-2-yl)sulfane in $\mathrm{CDCl}_{3}$.

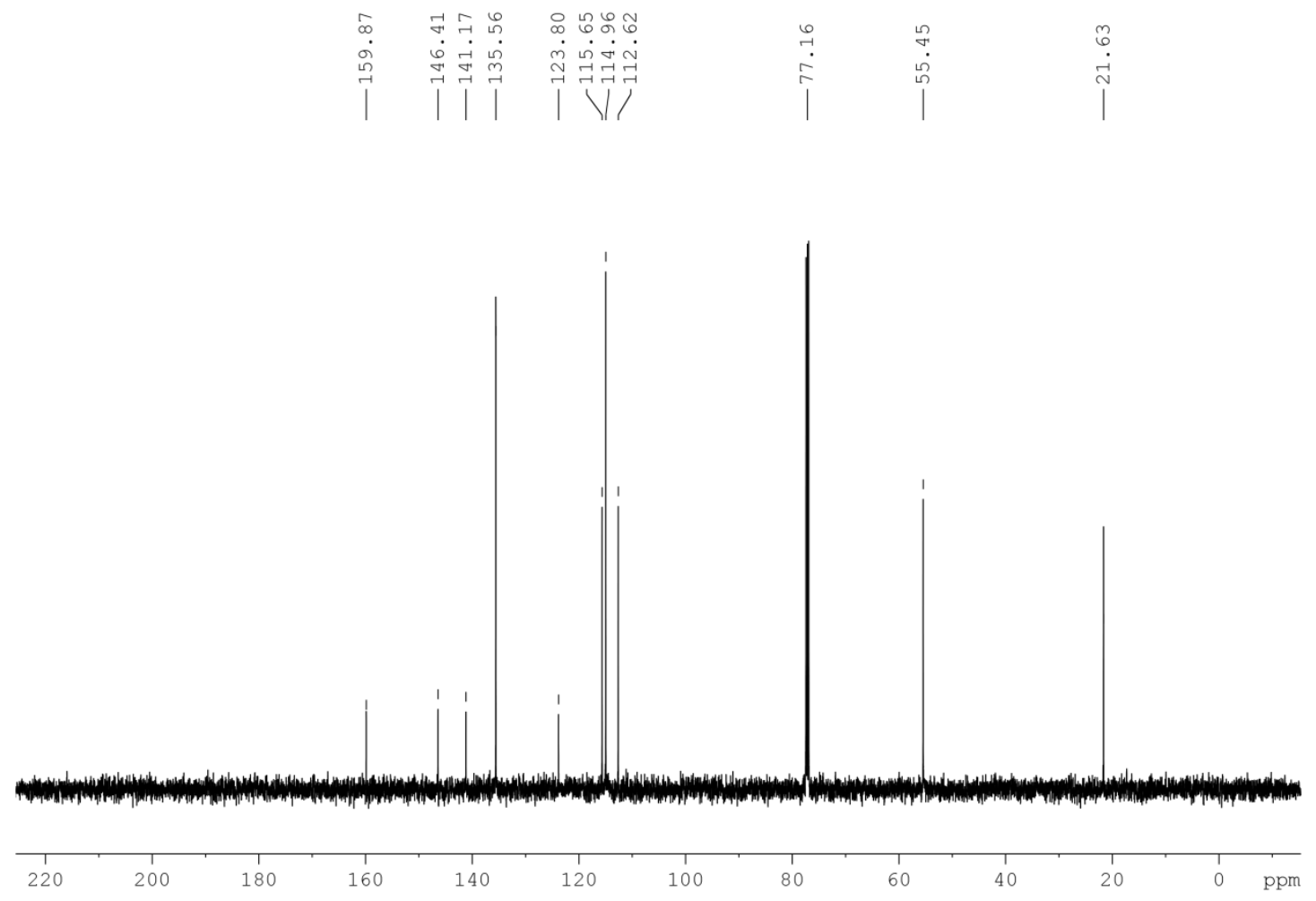

Figure S6. ${ }^{13} \mathrm{C}\left\{{ }^{1} \mathrm{H}\right\}$ NMR spectrum of 3c (4-methoxyphenyl)(3-methylbuta-1,3-dien-2yl)sulfane in $\mathrm{CDCl}_{3}$. 


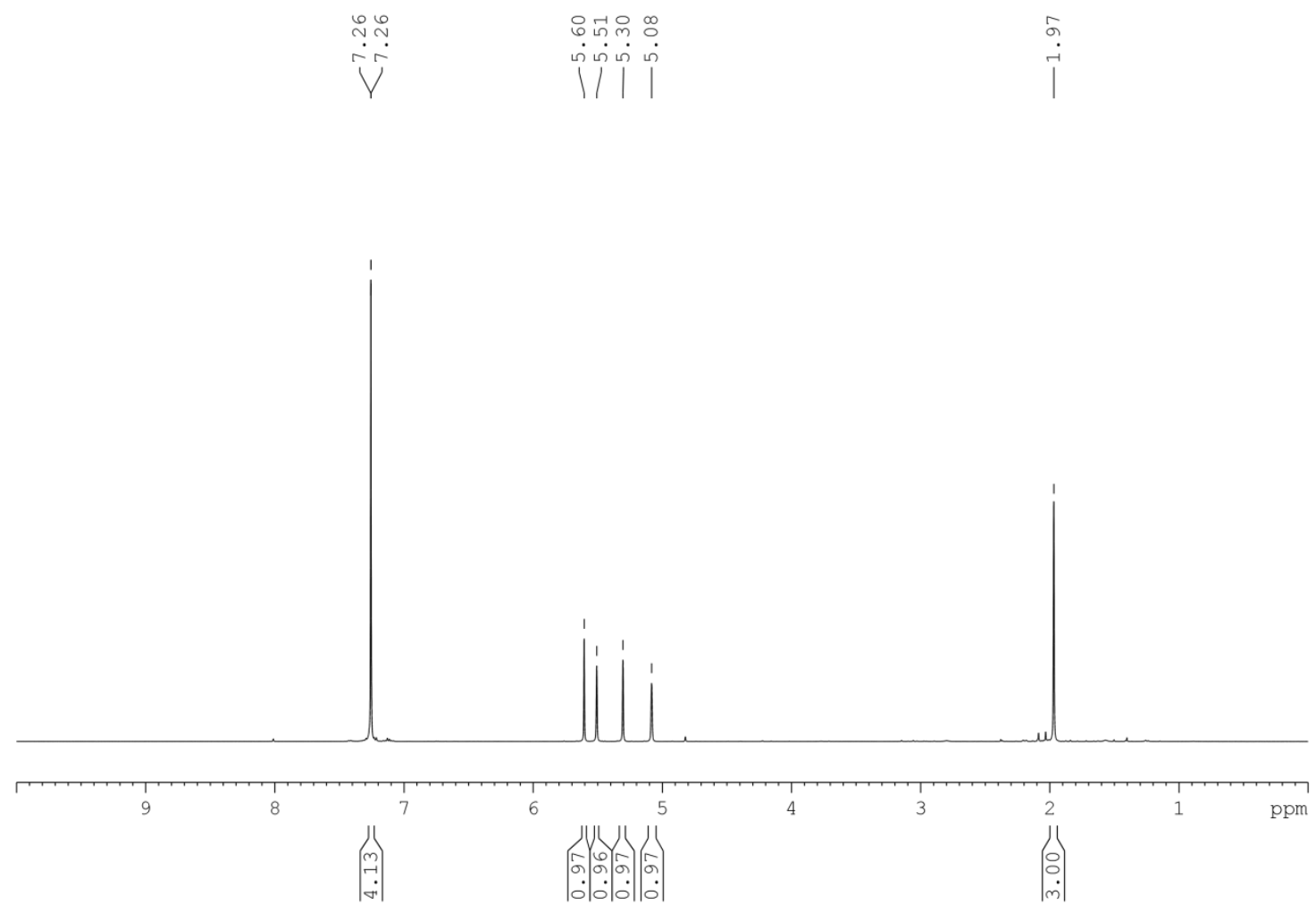

Figure S7. ${ }^{1} \mathrm{H}$ NMR spectrum of 3d (4-chlorophenyl)(3-methylbuta-1,3-dien-2-yl)sulfane in $\mathrm{CDCl}_{3}$.

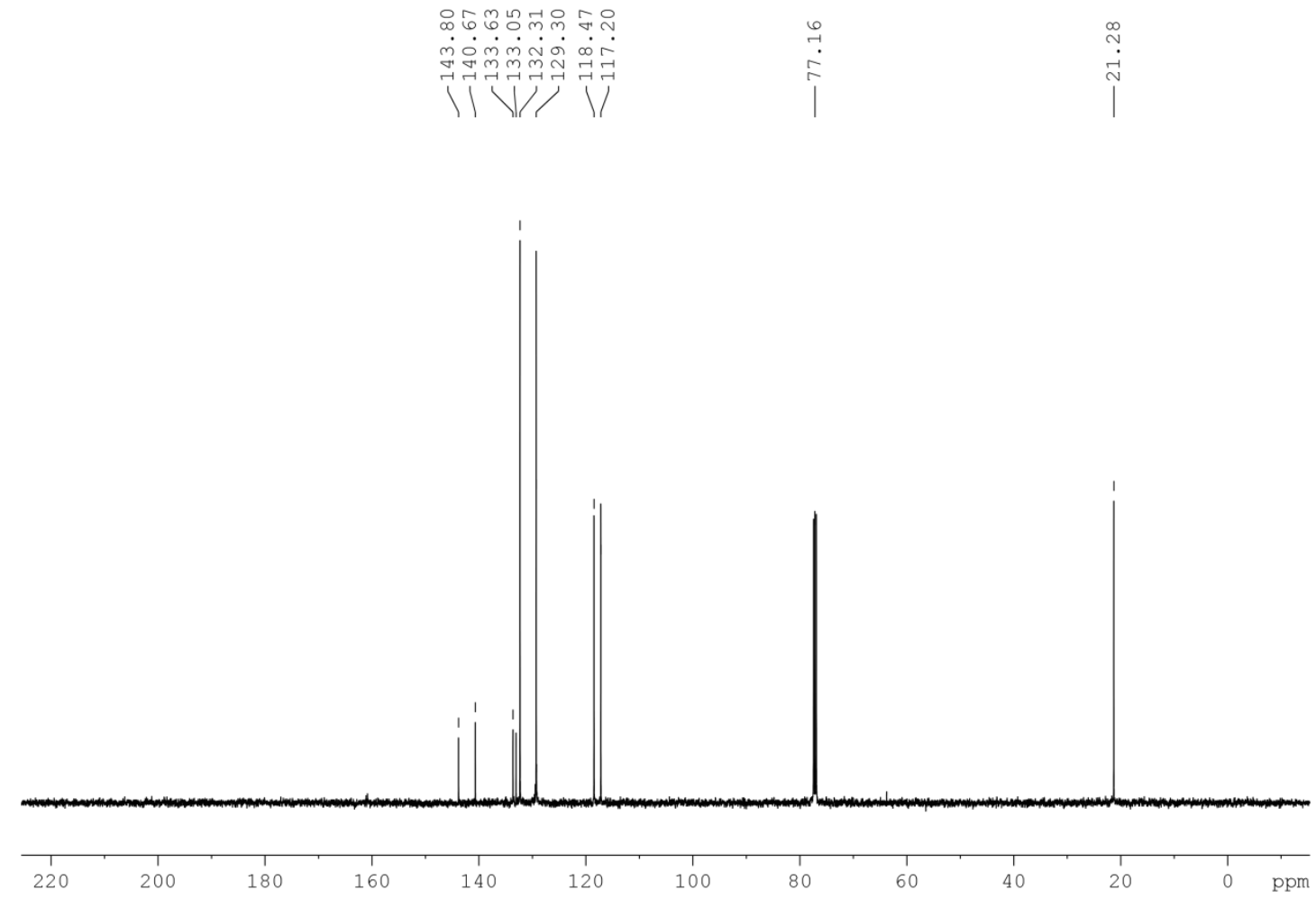

Figure S8. ${ }^{13} \mathrm{C}\left\{{ }^{1} \mathrm{H}\right\}$ NMR spectrum of 3d (4-chlorophenyl)(3-methylbuta-1,3-dien-2-yl)sulfane in $\mathrm{CDCl}_{3}$. 


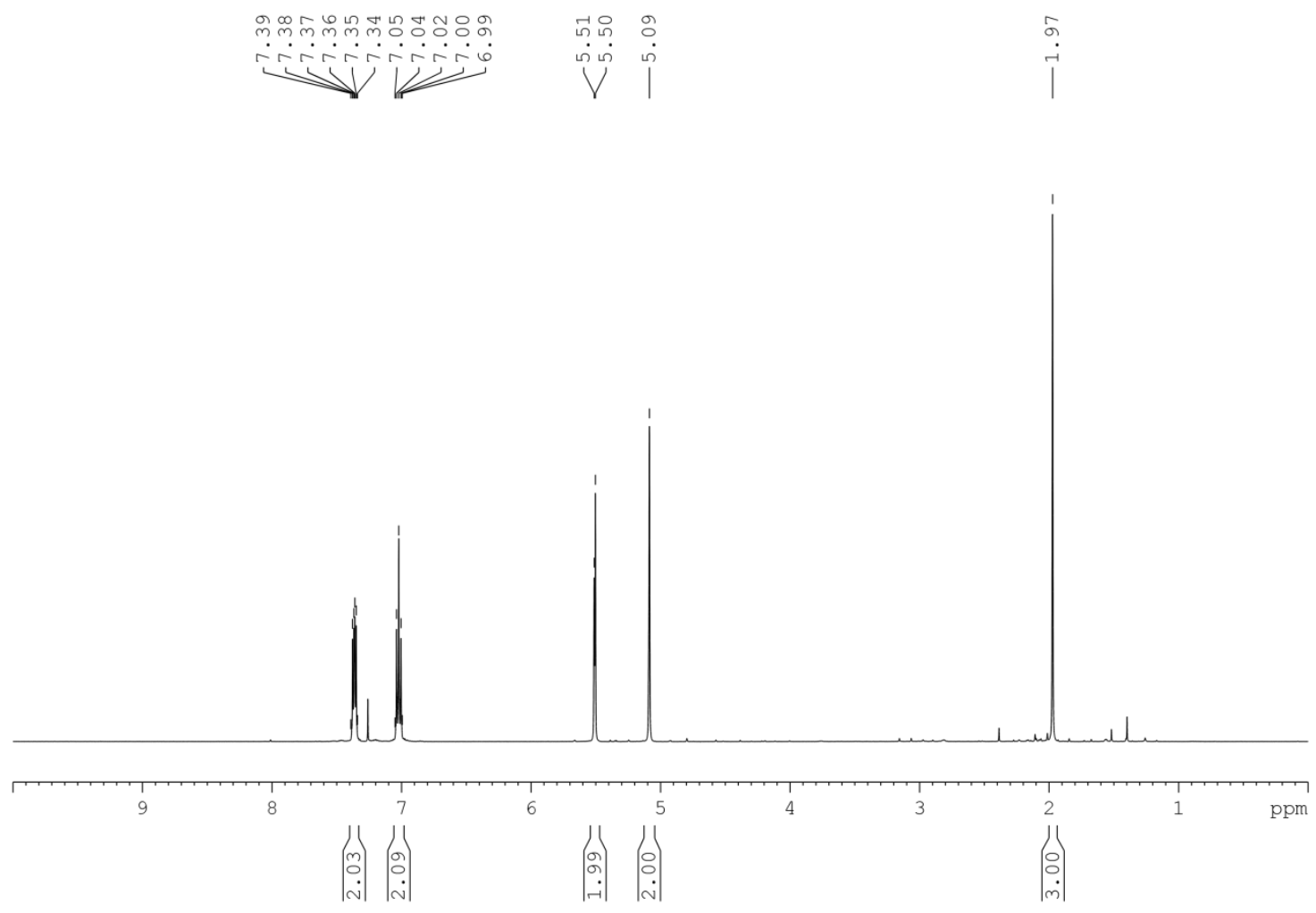

Figure S9. ${ }^{1} \mathrm{H}$ NMR spectrum of 3e (4-fluorophenyl)(3-methylbuta-1,3-dien-2-yl)sulfane in $\mathrm{CDCl}_{3}$.
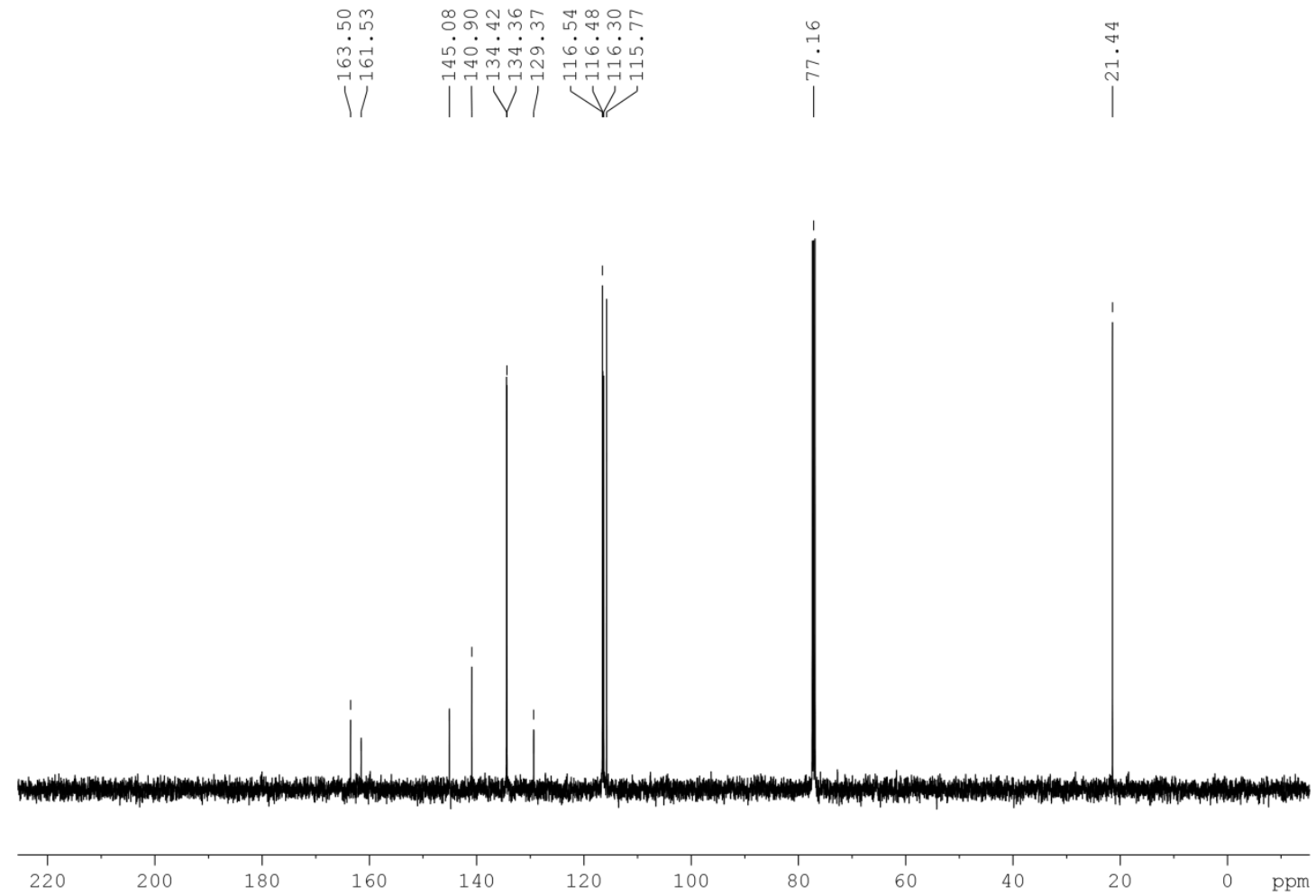

Figure S10. ${ }^{13} \mathrm{C}\left\{{ }^{1} \mathrm{H}\right\}$ NMR spectrum of 3e (4-fluorophenyl)(3-methylbuta-1,3-dien-2-yl)sulfane in $\mathrm{CDCl}_{3}$. 


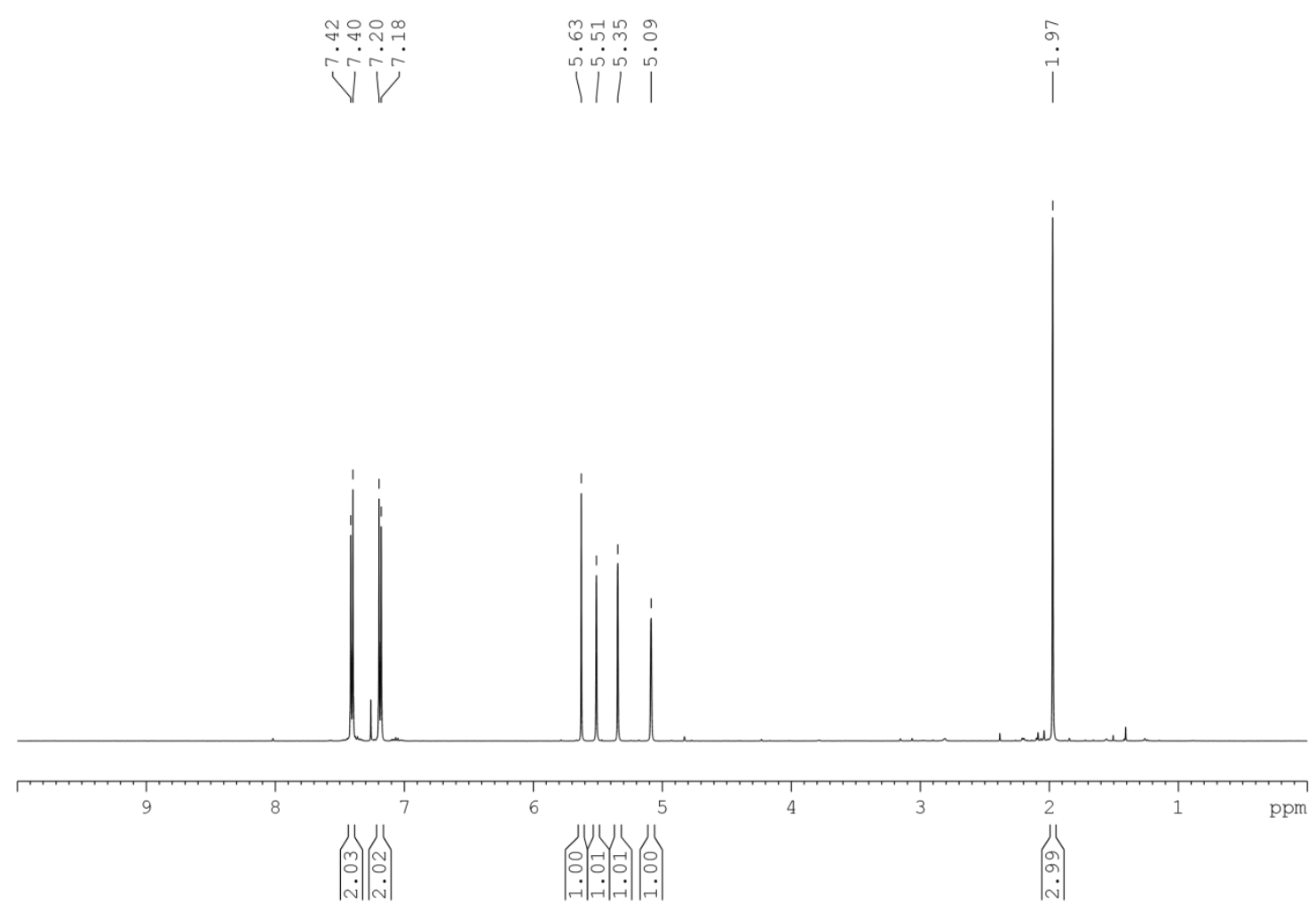

Figure S11. ${ }^{1} \mathrm{H}$ NMR spectrum of 3f (4-bromophenyl)(3-methylbuta-1,3-dien-2-yl)sulfane in $\mathrm{CDCl}_{3}$.

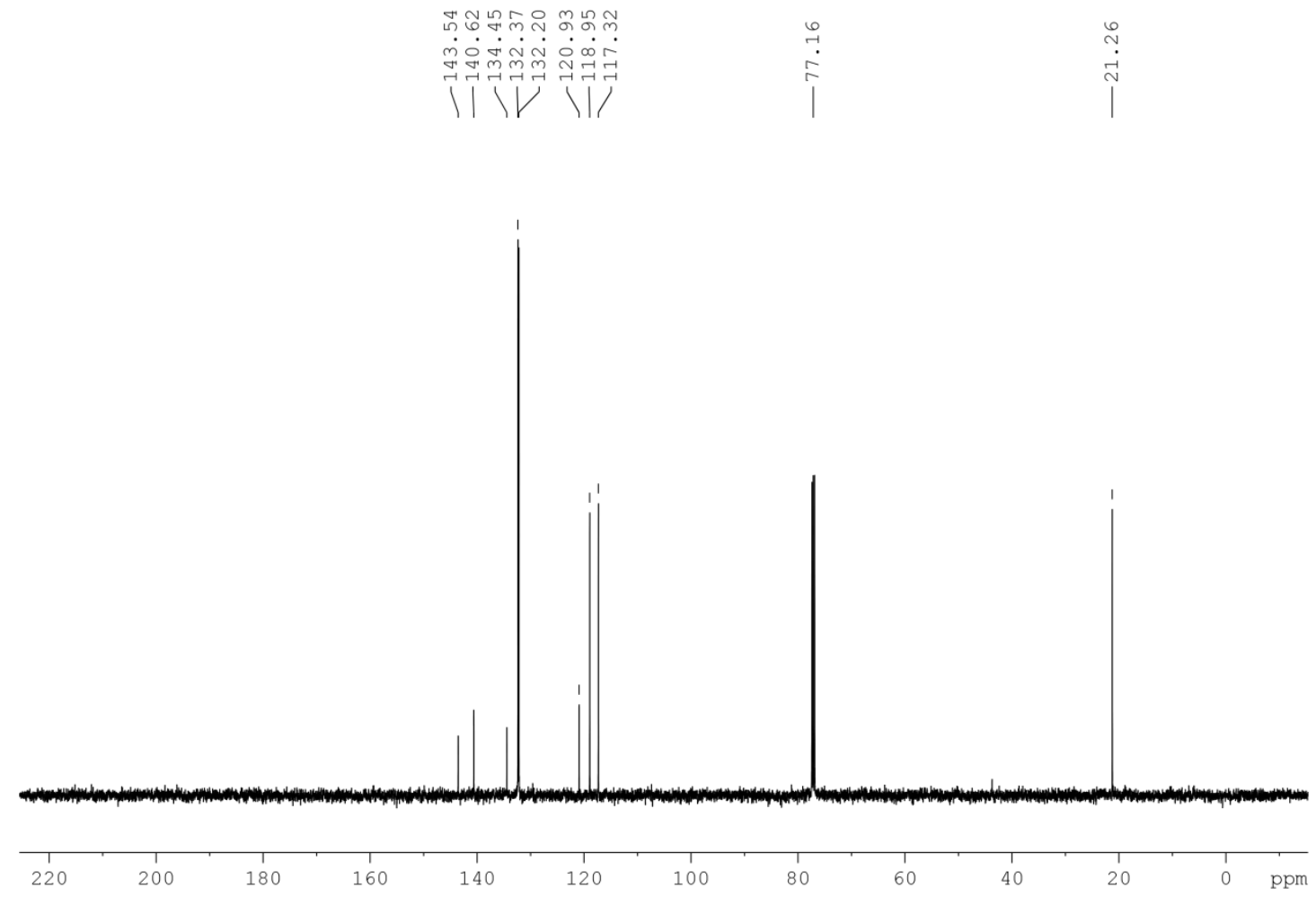

Figure S12. ${ }^{13} \mathrm{C}\left\{{ }^{1} \mathrm{H}\right\}$ NMR spectrum of $3 \mathbf{3}$ (4-bromophenyl)(3-methylbuta-1,3-dien-2-yl)sulfane in $\mathrm{CDCl}_{3}$. 


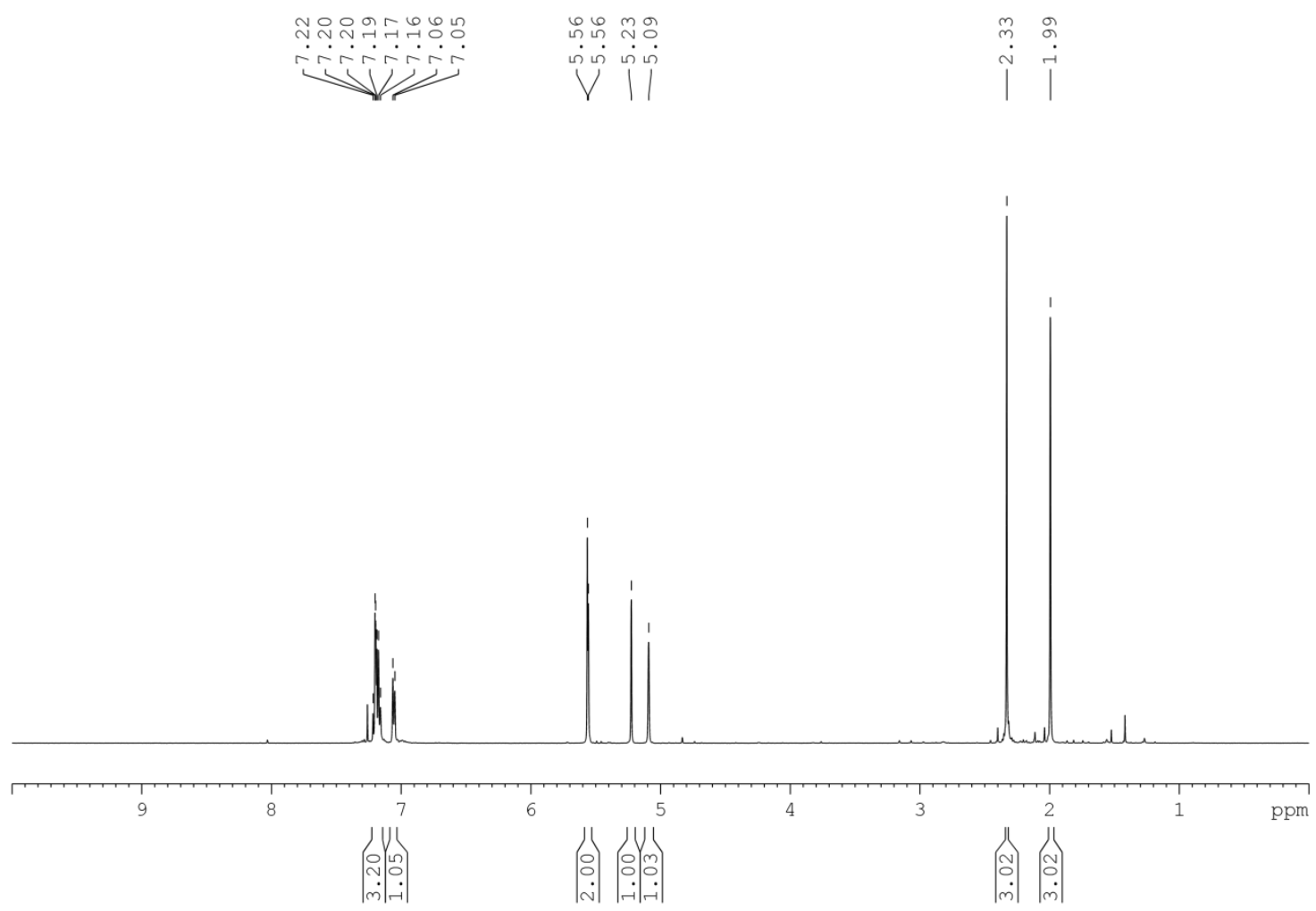

Figure S13. ${ }^{1} \mathrm{H}$ NMR spectrum of $\mathbf{3 g}$ (3-methylbuta-1,3-dien-2-yl)(m-tolyl)sulfane in $\mathrm{CDCl}_{3}$.

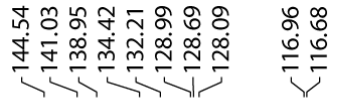

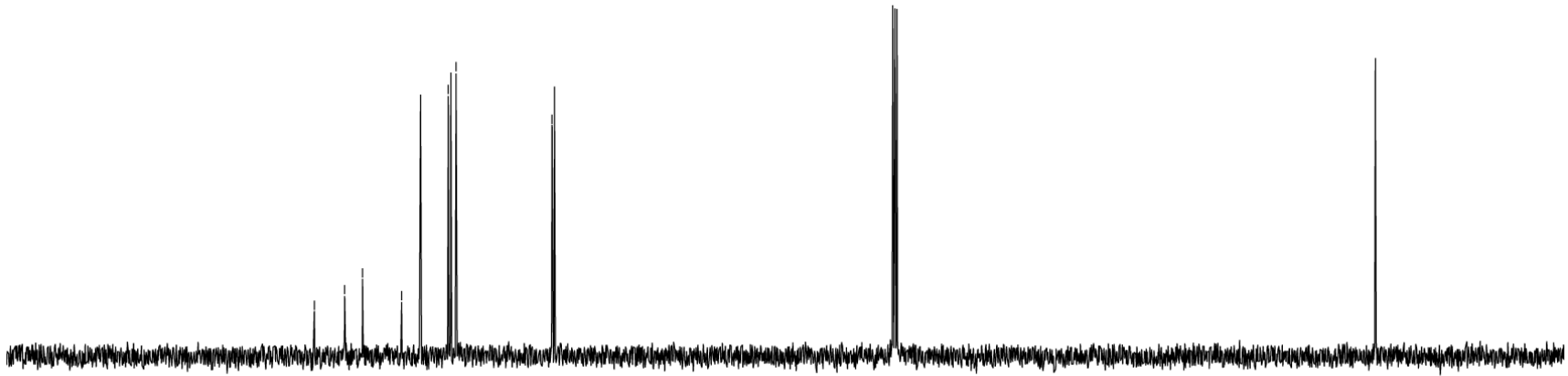

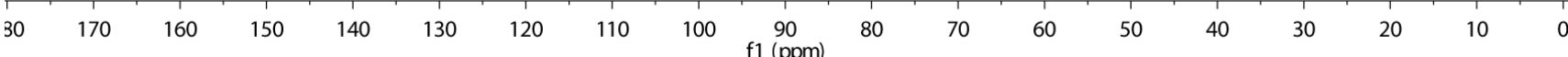

Figure S14. ${ }^{13} \mathrm{C}\left\{{ }^{1} \mathrm{H}\right\}$ NMR spectrum of $\mathbf{3 g}$ (3-methylbuta-1,3-dien-2-yl)(m-tolyl)sulfane in $\mathrm{CDCl}_{3}$. 


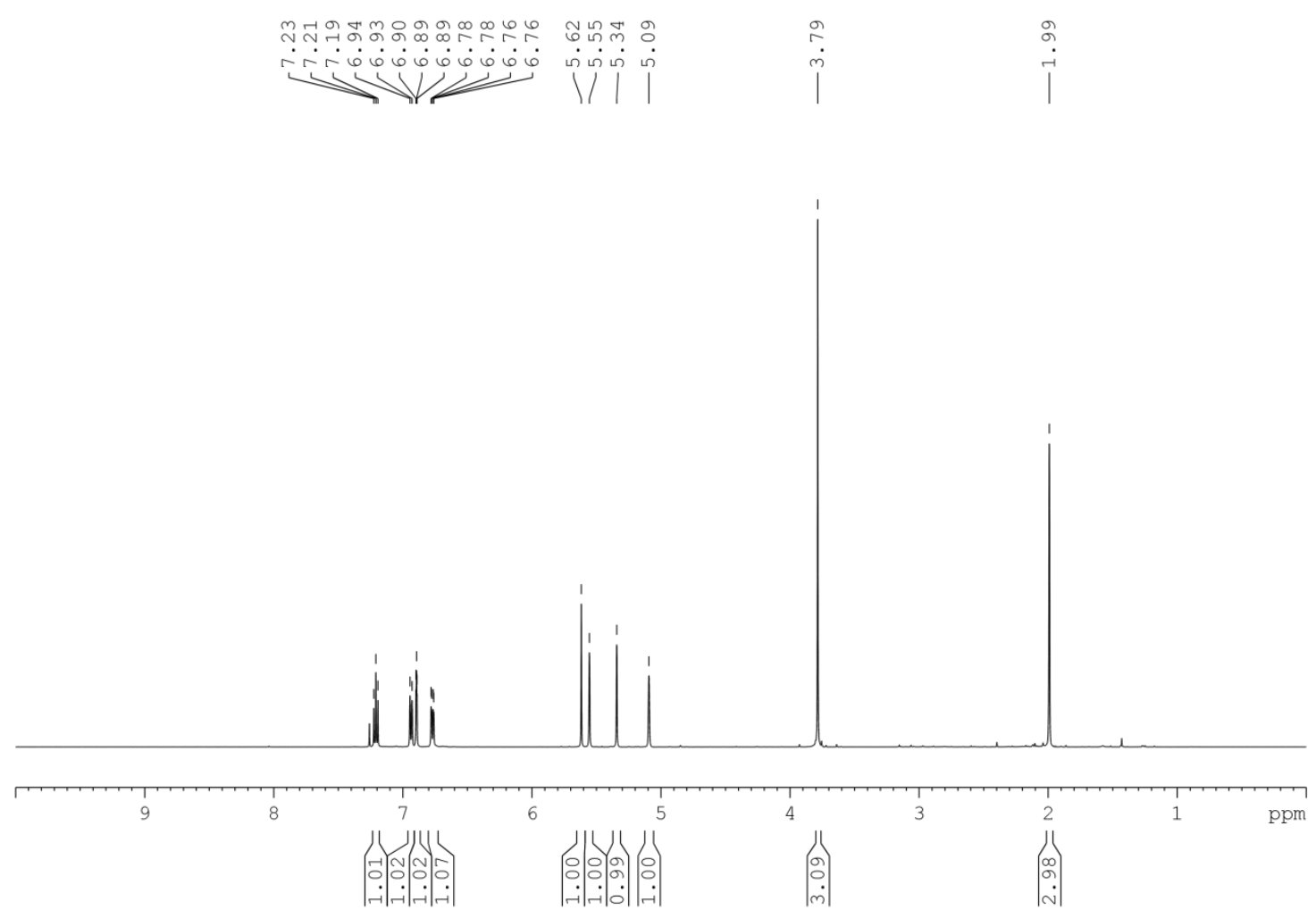

Figure S15. ${ }^{1} \mathrm{H}$ NMR spectrum of 3h (3-methoxyphenyl)(3-methylbuta-1,3-dien-2-yl)sulfane in $\mathrm{CDCl}_{3}$.
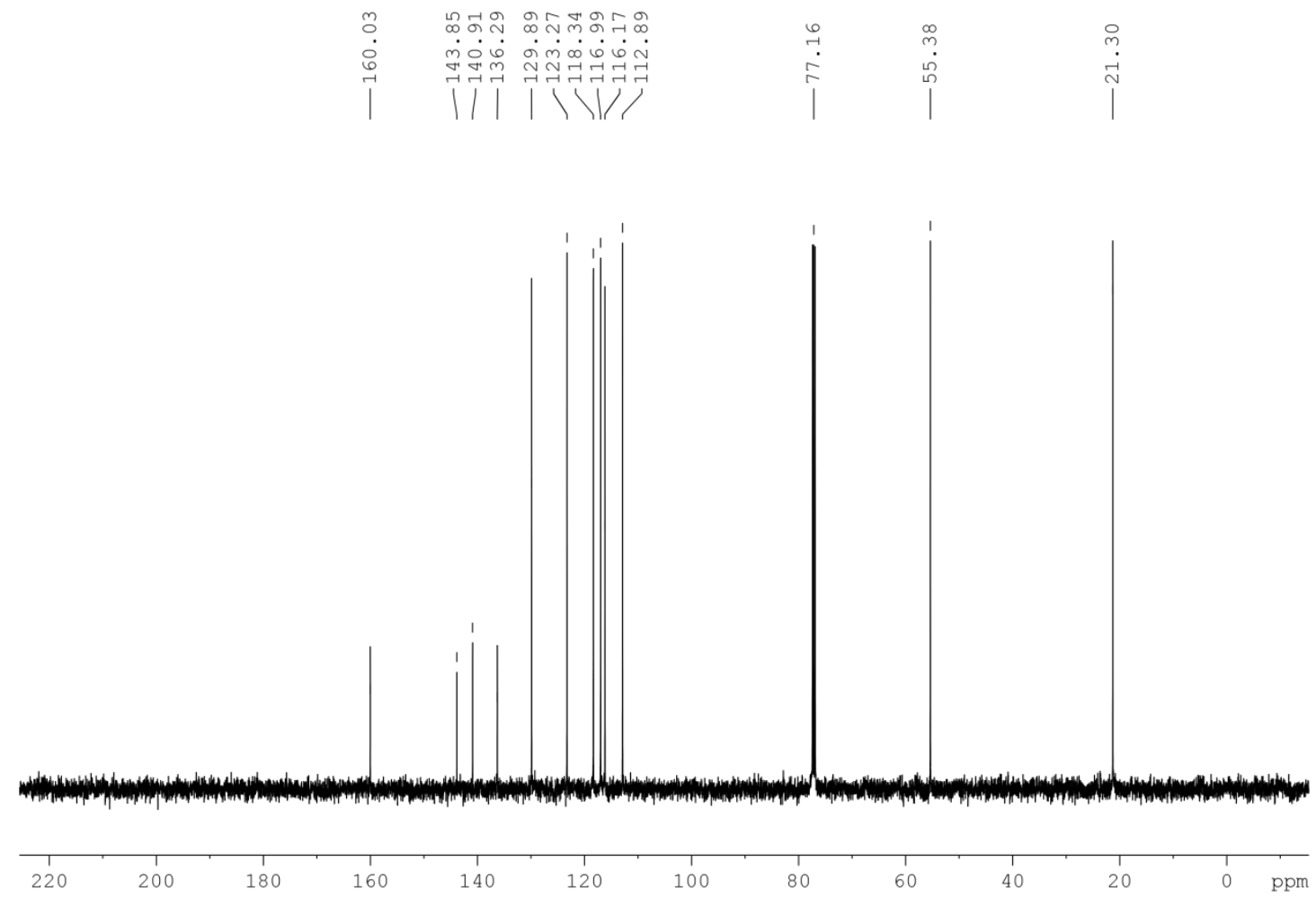

Figure S16. ${ }^{13} \mathrm{C}\left\{{ }^{1} \mathrm{H}\right\}$ NMR spectrum of $3 \mathrm{~h}$ (3-methoxyphenyl)(3-methylbuta-1,3-dien-2yl)sulfane in $\mathrm{CDCl}_{3}$. 


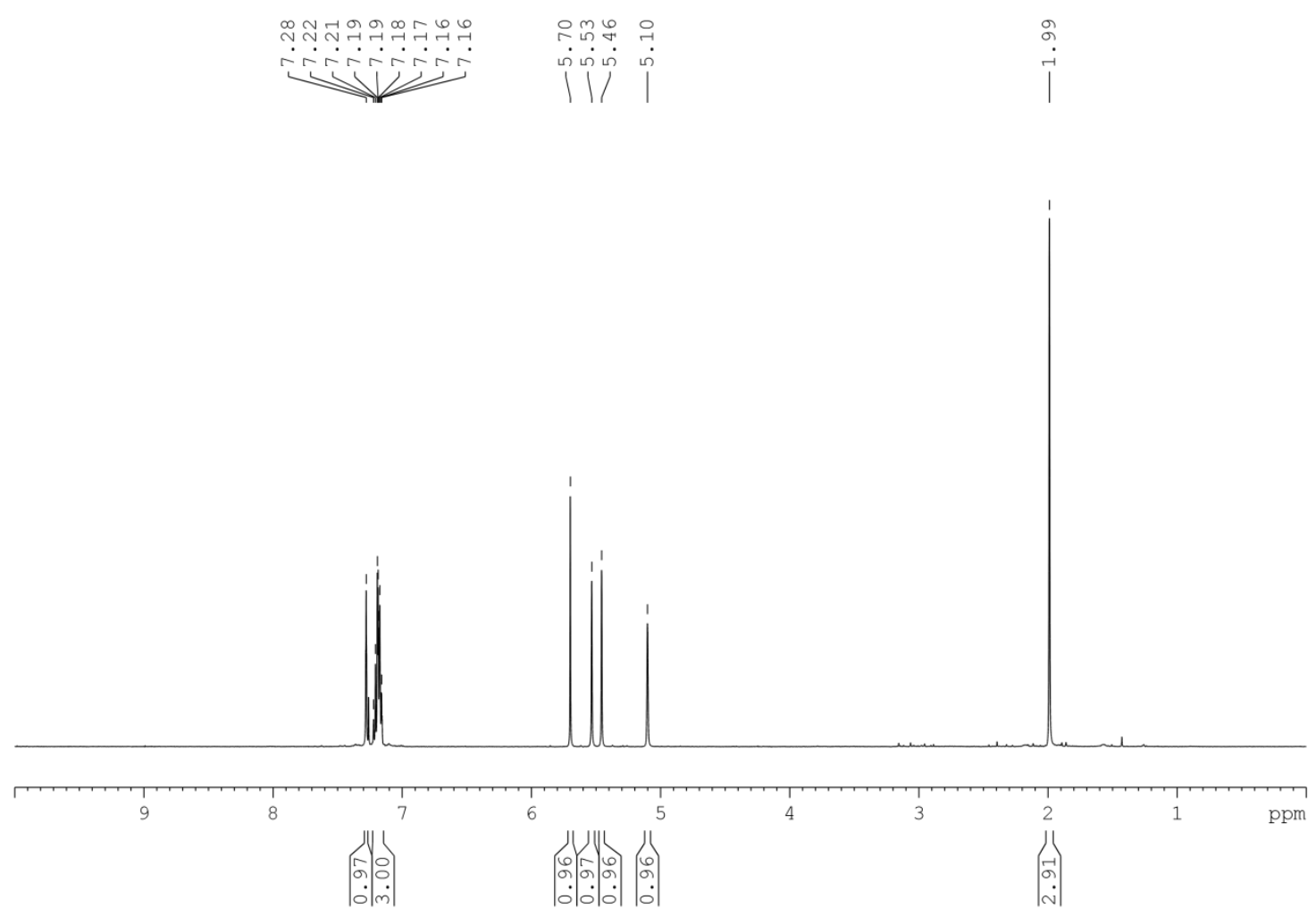

Figure S17. ${ }^{1} \mathrm{H}$ NMR spectrum of 3i (3-chlorophenyl)(3-methylbuta-1,3-dien-2-yl)sulfane in $\mathrm{CDCl}_{3}$.

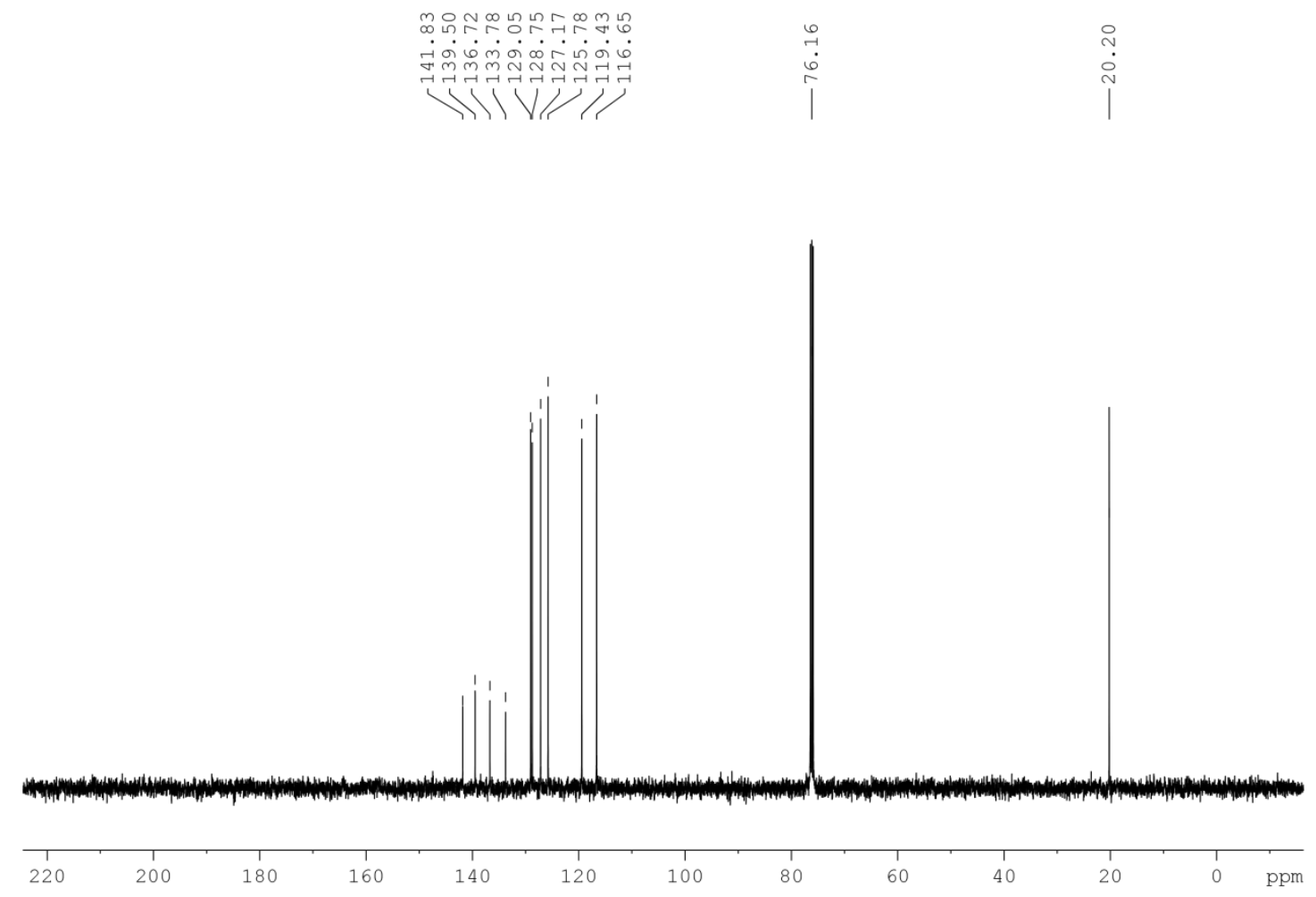

Figure S18. ${ }^{13} \mathrm{C}\left\{{ }^{1} \mathrm{H}\right\}$ NMR spectrum of 3i (3-chlorophenyl)(3-methylbuta-1,3-dien-2yl)sulfane in $\mathrm{CDCl}_{3}$. 


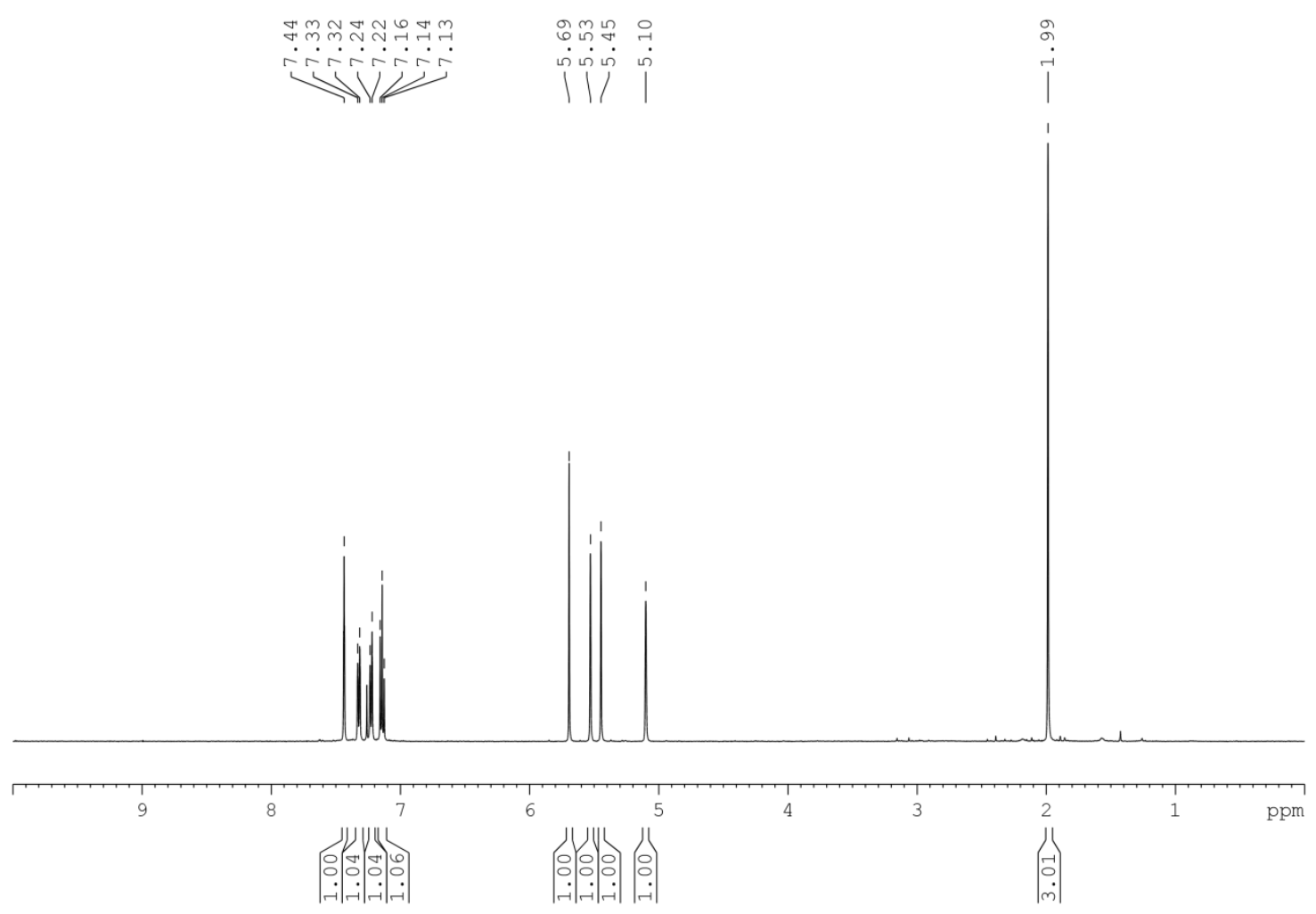

Figure S19. ${ }^{1} \mathrm{H}$ NMR spectrum of 3j (3-bromophenyl)(3-methylbuta-1,3-dien-2-yl)sulfane in $\mathrm{CDCl}_{3}$.

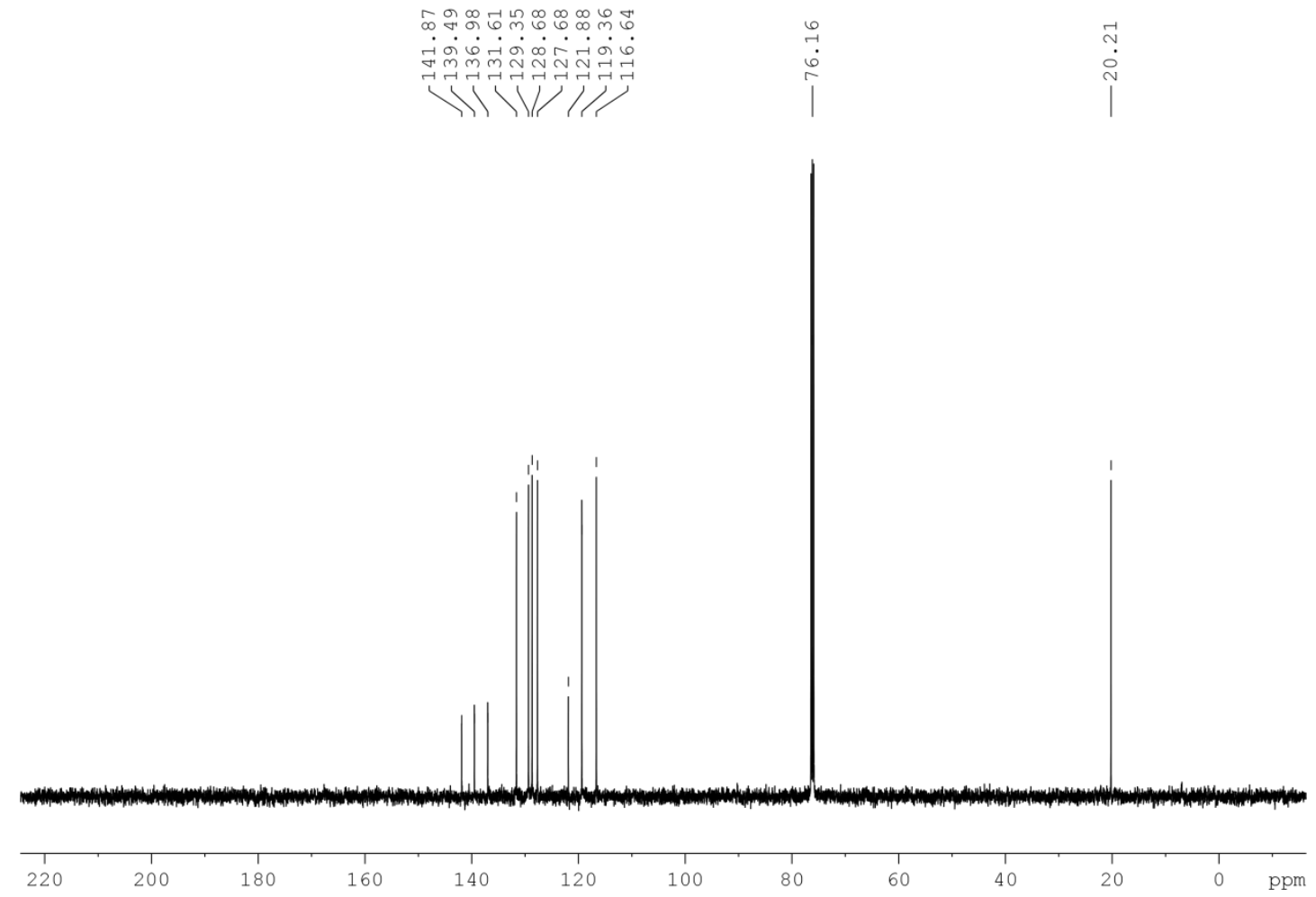

Figure S20. ${ }^{13} \mathrm{C}\left\{{ }^{1} \mathrm{H}\right\}$ NMR spectrum of 3j (3-bromophenyl)(3-methylbuta-1,3-dien-2-yl)sulfane in $\mathrm{CDCl}_{3}$. 


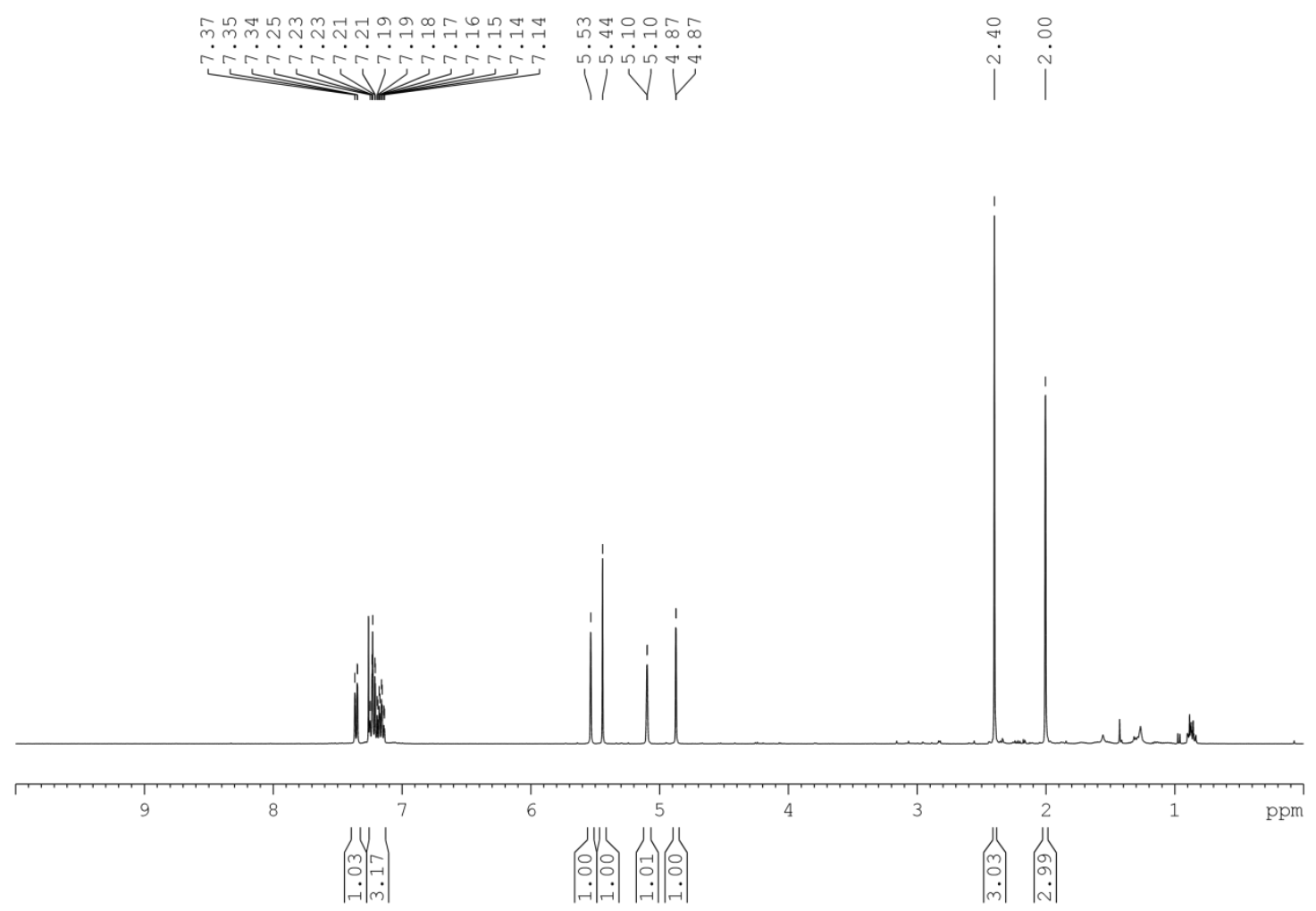

Figure S21. ${ }^{1} \mathrm{H}$ NMR spectrum of 3k 3-(methylbuta-1,3-dien-2-yl)(o-tolyl)sulfane in $\mathrm{CDCl}_{3}$.
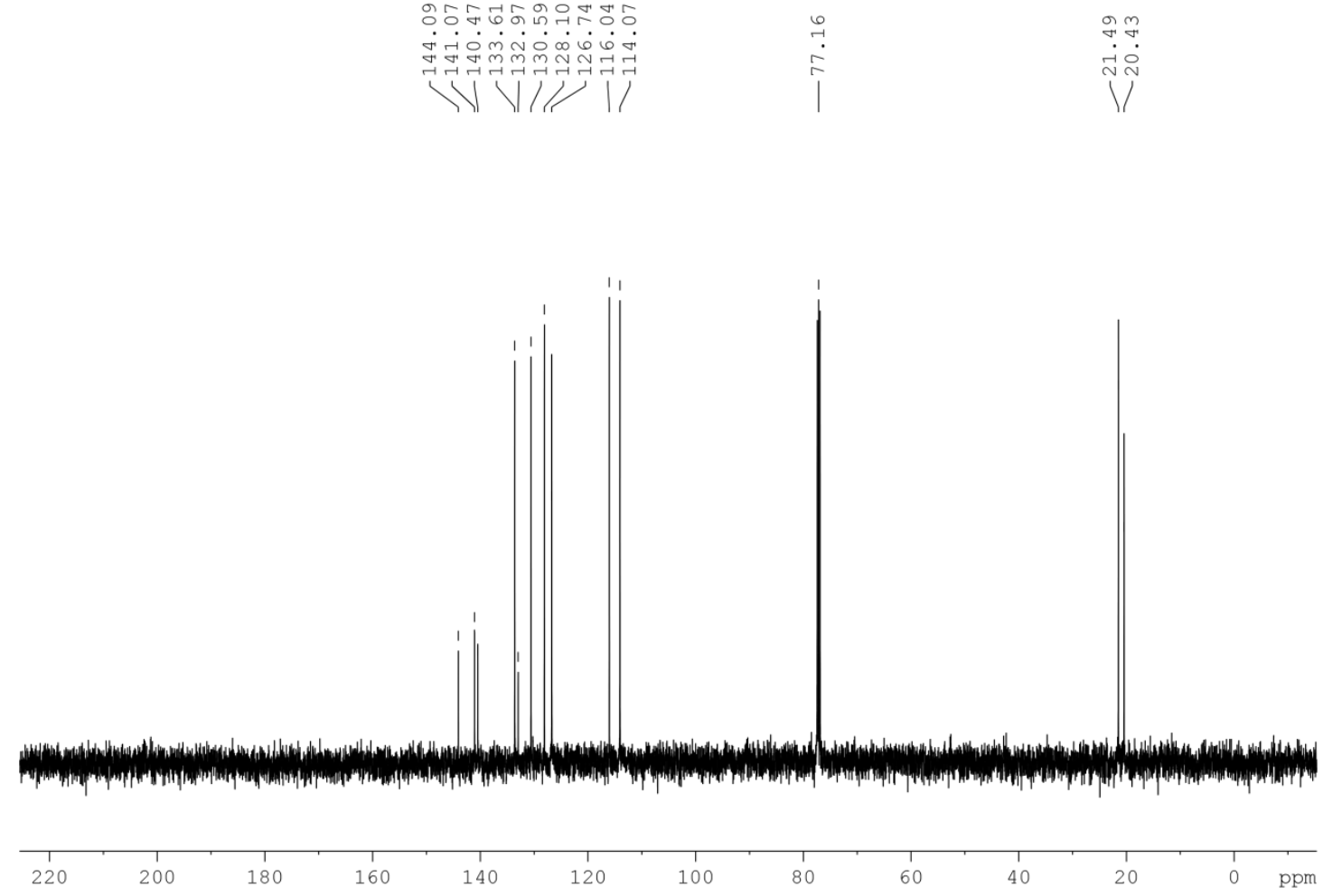

Figure S22. ${ }^{13} \mathrm{C}\left\{{ }^{1} \mathrm{H}\right\}$ NMR spectrum of 3k 3-(methylbuta-1,3-dien-2-yl)(o-tolyl)sulfane in $\mathrm{CDCl}_{3}$. 


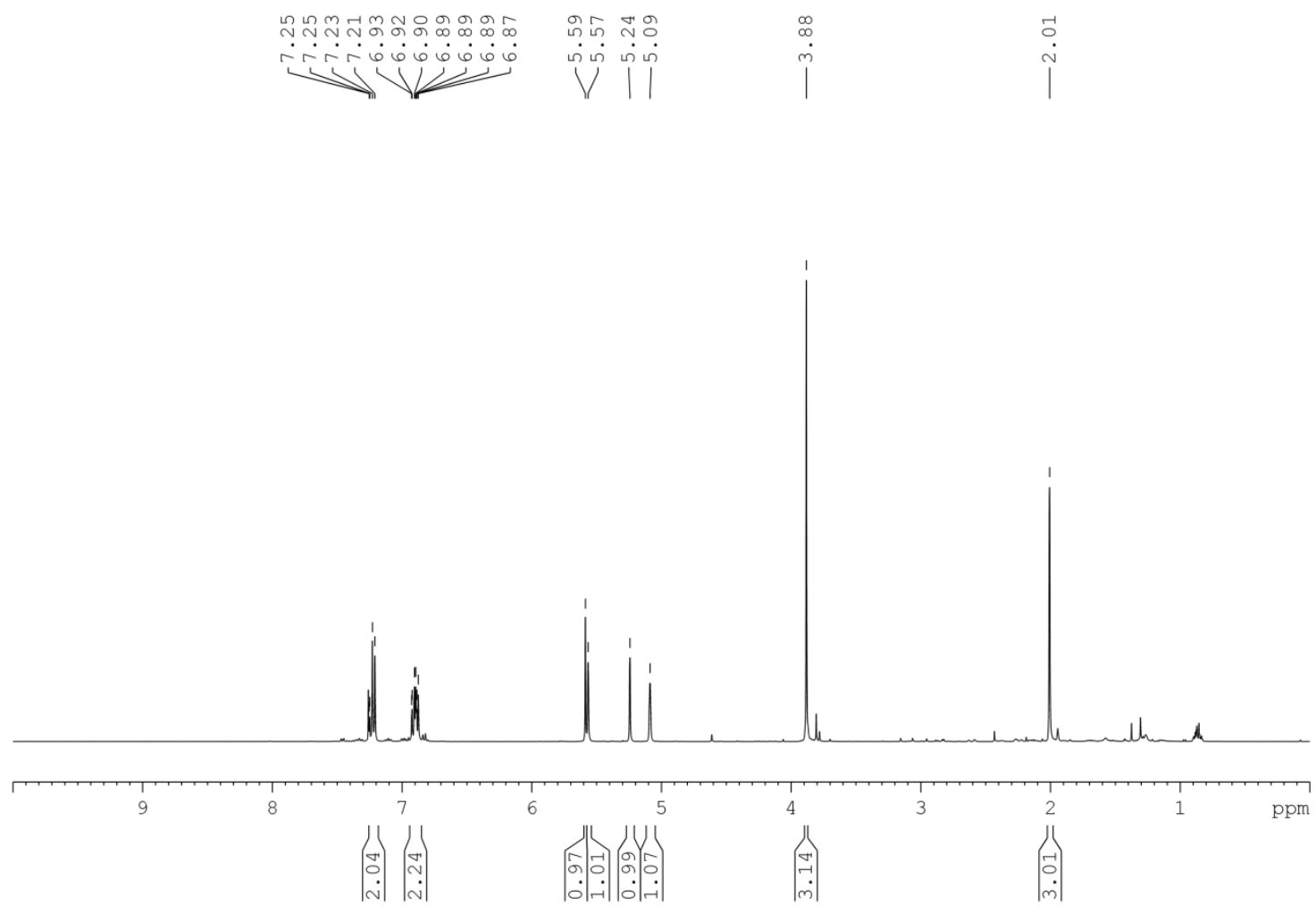

Figure S23. ${ }^{1} \mathrm{H}$ NMR spectrum of 3I (2-methoxyphenyl)(3-methylbuta-1,3-dien-2-yl)sulfane in $\mathrm{CDCl}_{3}$.
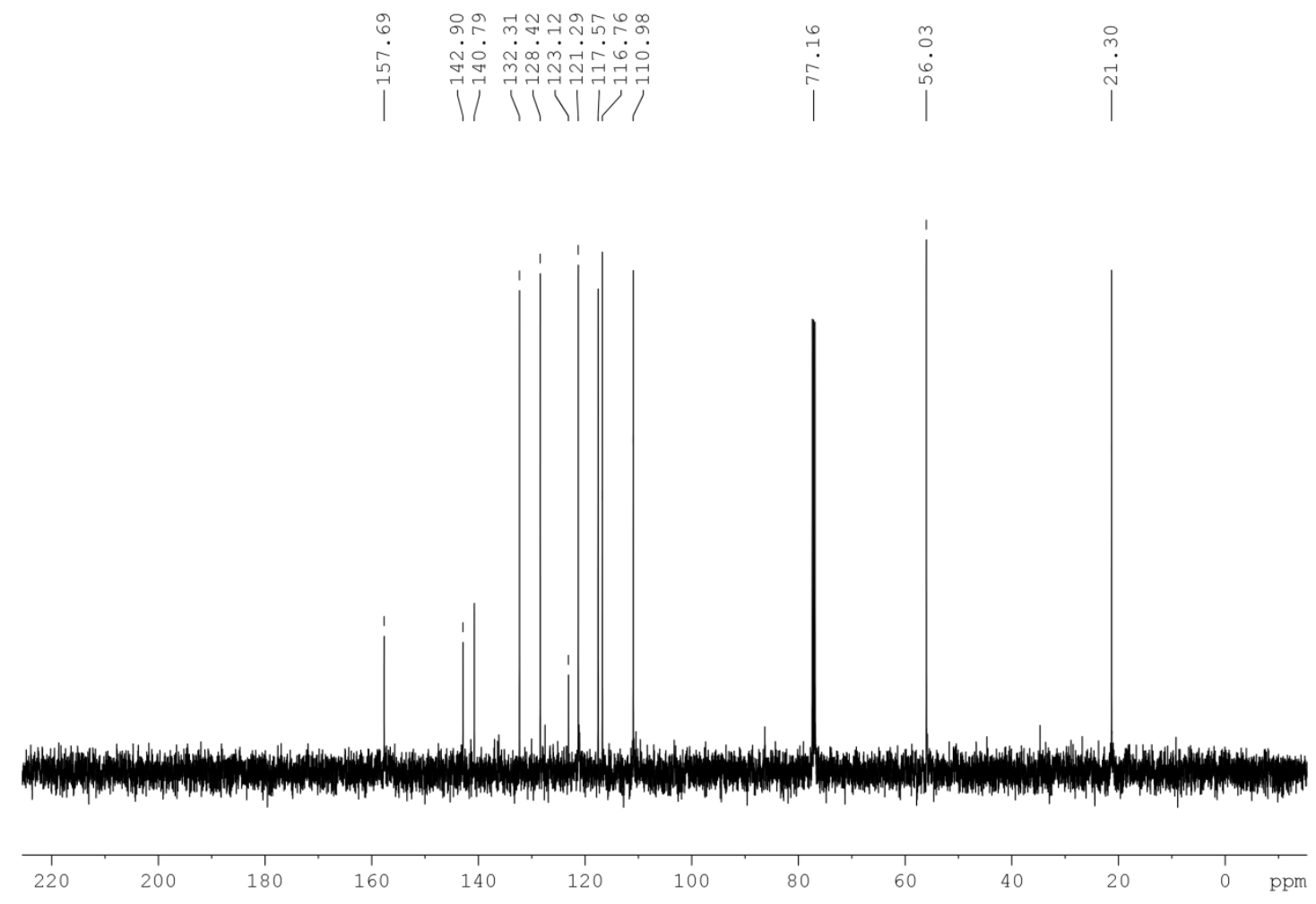

Figure S24. ${ }^{13} \mathrm{C}\left\{{ }^{1} \mathrm{H}\right\}$ NMR spectrum of 3I (2-methoxyphenyl)(3-methylbuta-1,3-dien-2yl)sulfane in $\mathrm{CDCl}_{3}$. 


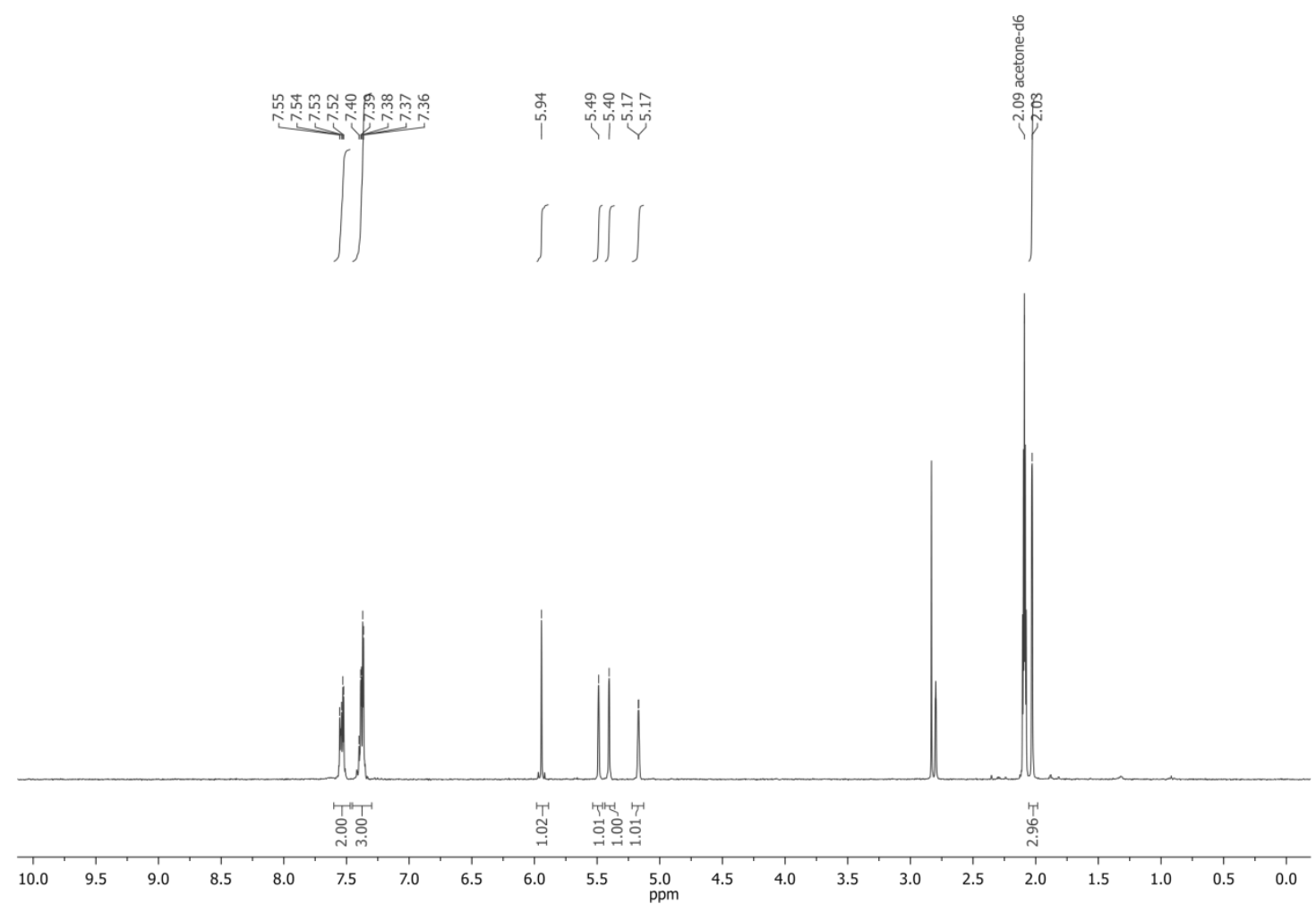

Figure S25. ${ }^{1} \mathrm{H}$ NMR spectrum of 3m (3-methylbuta-1,3-dien-2-yl)(phenyl)selane in acetone$d_{6}$.

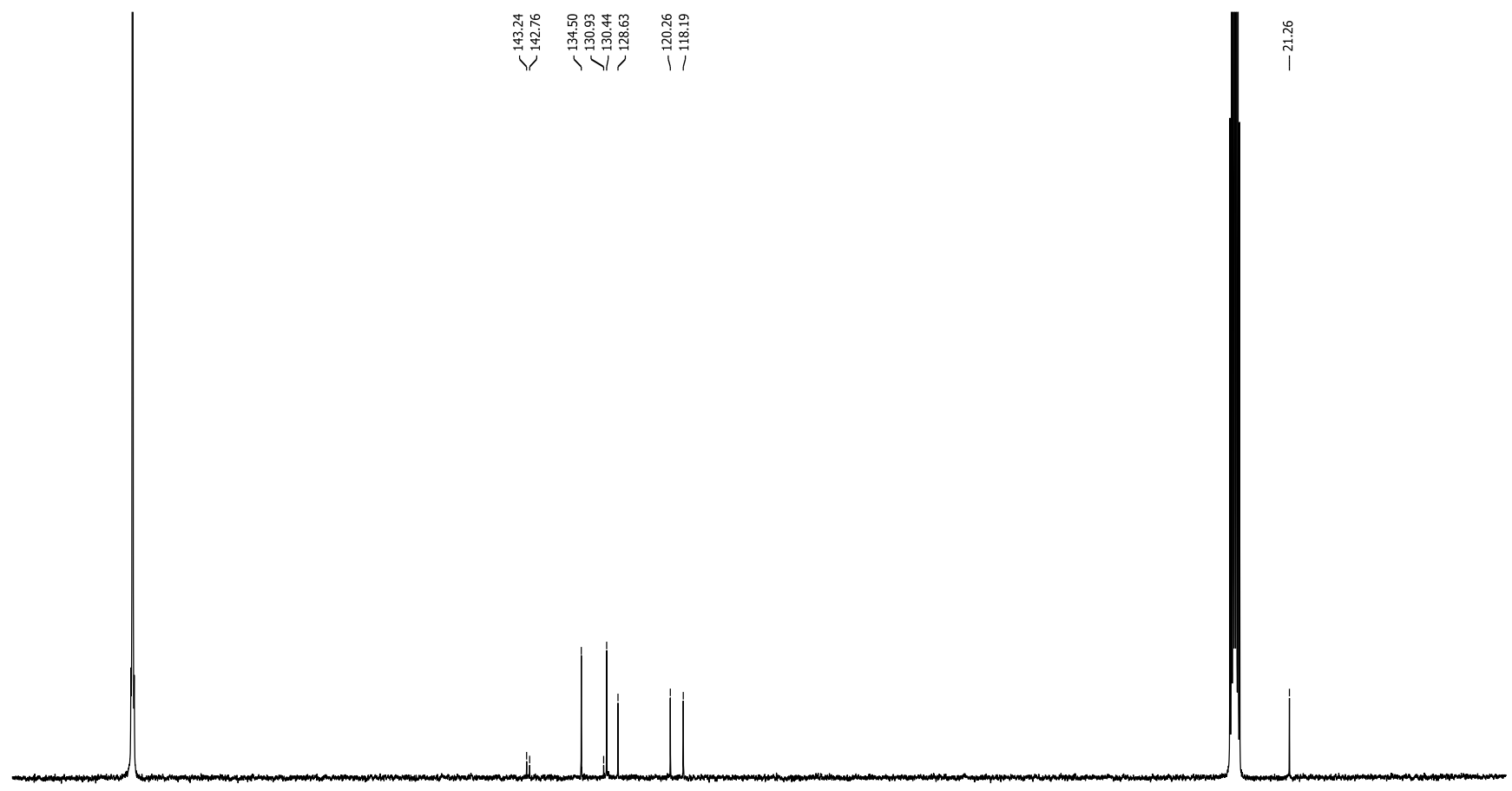

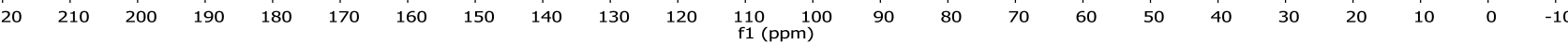

Figure S26. ${ }^{13} \mathrm{C}\left\{{ }^{1} \mathrm{H}\right\}$ NMR spectrum of $3 \mathrm{~m}$ (3-methylbuta-1,3-dien-2-yl)(phenyl)selane in acetone- $d_{6}$. 


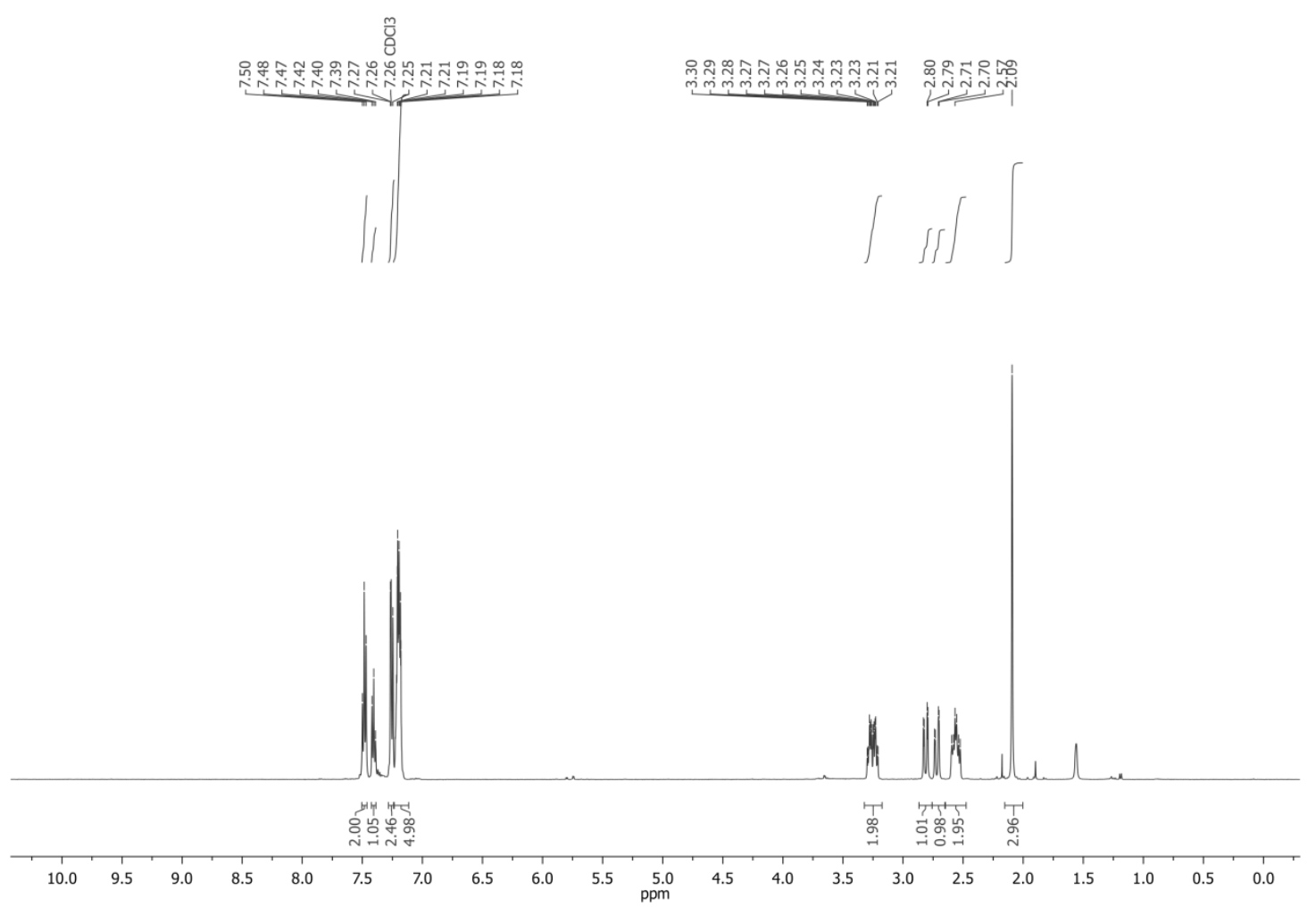

Figure S27. ${ }^{1} \mathrm{H}$ NMR spectrum of 4 5-methyl-2-phenyl-6-(phenylthio)-3a,4,7,7a-tetrahydro$1 \mathrm{H}$-isoindole-1,3(2H)-dione in $\mathrm{CDCl}_{3}$.

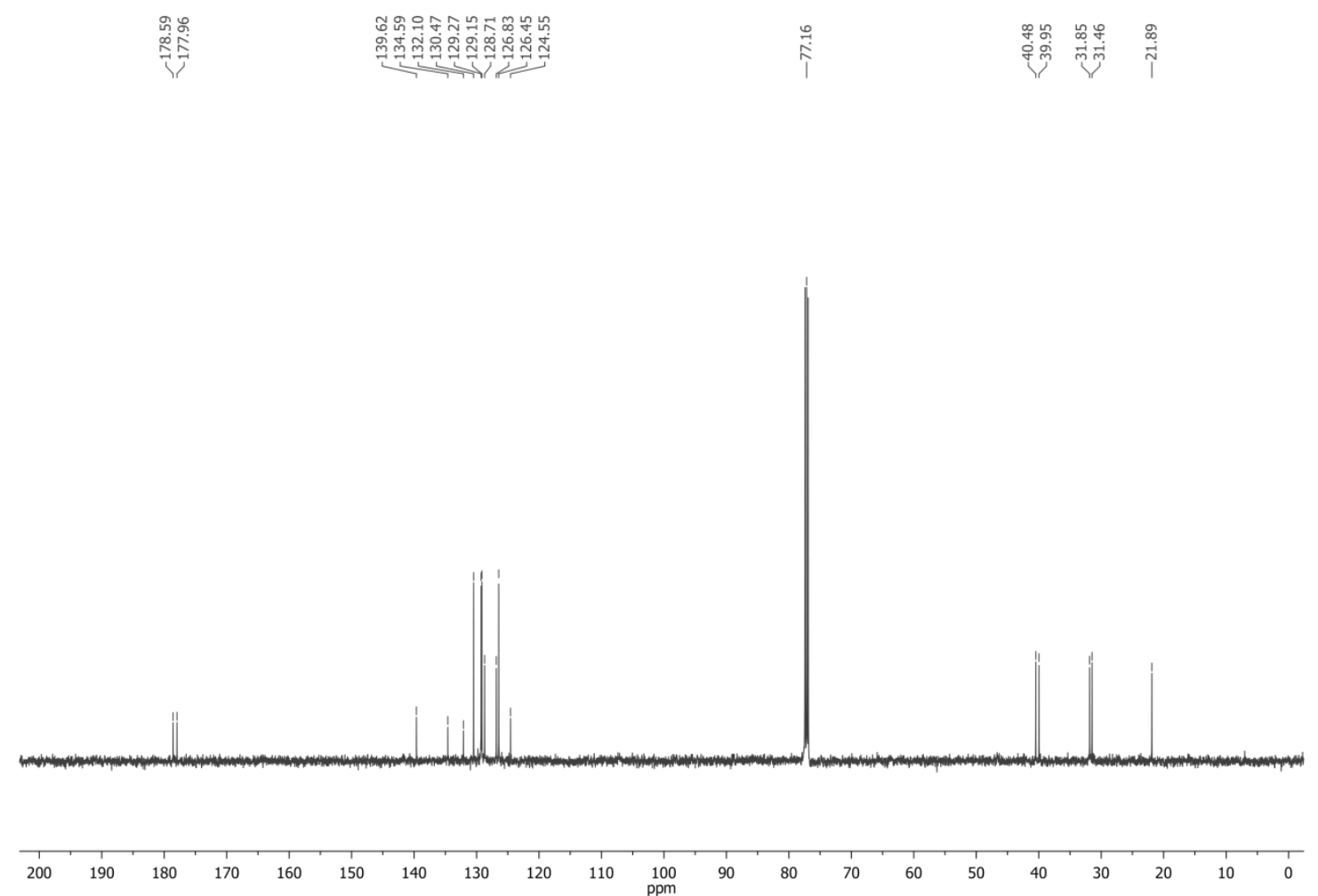

Figure S28. ${ }^{13} \mathrm{C}\left\{{ }^{1} \mathrm{H}\right\}$ NMR spectrum of 4 5-methyl-2-phenyl-6-(phenylthio)-3a,4,7,7atetrahydro-1H-isoindole-1,3(2H)-dione in $\mathrm{CDCl}_{3}$. 


\section{References}

[1] R. A. Sheldon, Green Chem. 2017, 19, 18-43.

[2] F. Roschangar, R. A. Sheldon, C. H. Senanayake, Green Chem. 2015, 17, 752-768.

[3] V. P. Ananikov, N. V. Orlov, I. P. Beletskaya, Organometallics 2006, 25, 1970-1977.

[4] V. P. Ananikov, S. S. Zalesskiy, N. V. Orlov, I. P. Beletskaya, Russ. Chem. Bull. 2006, 55, 2109-2113.

[5] H. Kuniyasu, A. Ogawa, K. Sato, I. Ryu, N. Kambe, N. Sonoda, J. Am. Chem. Soc. 1992, 114, 5902-5903.

[6] W. C. Still, M. Kahn, A. Mitra, J. Org. Chem. 1978, 43, 2923-2925.

[7] M. Casey, J. Leonard, B. Lygo, G. Procter, Advanced Practical Organic Chemistry, Springer US, Boston, MA, 1990.

[8] R. Jacquot, Method for Preparing Fluorinated Compounds, 2004, US2004054234.

[9] H. Gupta, C. Gupta, K. Kumar, R. Saxena, R. Anand, Processes for Producing 1,1,1,5,5,5 Hexafluoroacetyl Acetone, 2015, WO2015118561.

[10] D. B. Eremin, D. A. Boiko, E. V. Borkovskaya, V. N. Khrustalev, V. M. Chernyshev, V. P. Ananikov, Catal. Sci. Technol. 2018, 8, 3073-3080.

[11] E. S. Degtyareva, J. V. Burykina, A. N. Fakhrutdinov, E. G. Gordeev, V. N. Khrustalev, V. P. Ananikov, ACS Catal. 2015, 5, 7208-7213.

[12] T. M. Trnka, J. P. Morgan, M. S. Sanford, T. E. Wilhelm, M. Scholl, T.-L. Choi, S. Ding, M. W. Day, R. H. Grubbs, J. Am. Chem. Soc. 2003, 125, 2546-58.

[13] L. Hintermann, Beilstein J. Org. Chem. 2007, 3, 22.

[14] M. P. Cava, A. A. Deana, K. Muth, M. J. Mitchel, Org. Synth. 1961, 41, 93. 